\title{
PARACONTROLLED DISTRIBUTIONS AND THE 3-DIMENSIONAL STOCHASTIC QUANTIZATION EQUATION
}

\author{
By RÉmi CATELliER ${ }^{1}$ AND KHALil CHOUK $^{2}$ \\ Université Paris-Dauphine
}

\begin{abstract}
We prove the existence and uniqueness of a local in time solution to the periodic $\Phi_{3}^{4}$ model of stochastic quantisation using the method of paracontrolled distributions introduced recently by M. Gubinelli, P. Imkeller and N. Perkowski in [Forum Math., Pi 3 (2015) e6].
\end{abstract}

\section{CONTENTS}

1. Introduction . . . . . . . . . . . . . . . . . . . . . . . . . . . . . . 2622

Plan of the paper . . . . . . . . . . . . . . . . . . . . . 2626

2. Basic tools, description of the proof and rough distributions f . . . . . . . . . 2626

2.1. Besov spaces and paradifferential calculus . . . . . . . . . . . . . . . . . 2626

2.2. Description of the strategy and renormalized equation . . . . . . . . . . . . . . . . 2629

2.3. Rough distributions . . . . . . . . . . . . . . . . . . . . . . . 2635

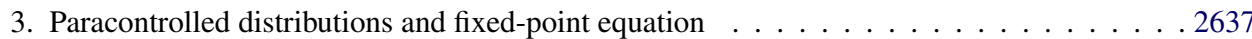

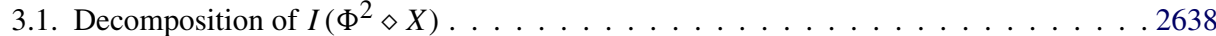

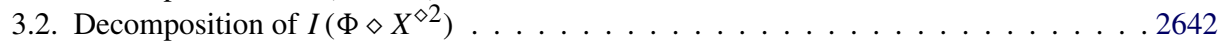

3.3. Fixed-point procedure . . . . . . . . . . . . . . . . . . . . . . . . . . . . . . 2649

4. Renormalization and construction of the rough distribution . . . . . . . . . . . . 2653

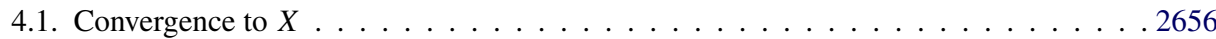

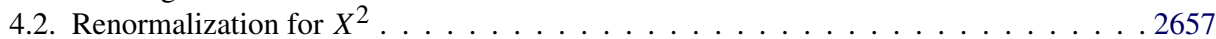

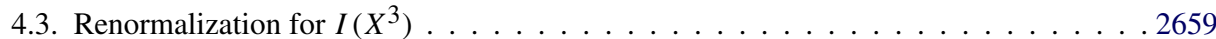

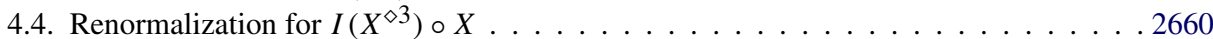

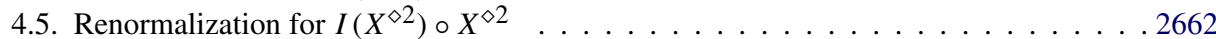

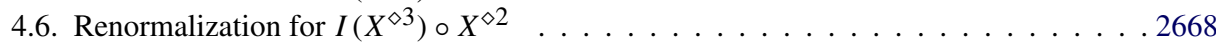

Terms in the first chaos . . . . . . . . . . . . . . . . . . . . . . . . . . . . 2669

Terms in the third chaos . . . . . . . . . . . . . . . . . . . . 2671

Terms in the fifth chaos . . . . . . . . . . . . . . . . . . . . . . . . 2673

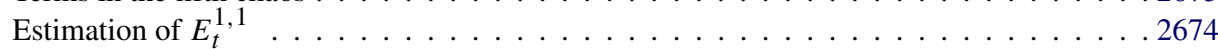

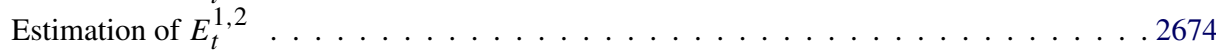

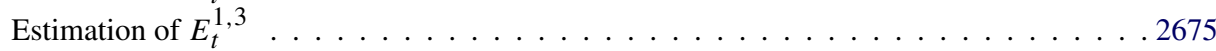

Appendix: A commutation lemma . . . . . . . . . . . . . . . . . . . . . 2676

References . . . . . . . . . . . . . . . . . . . . . . . . . . 2679

Received November 2013; revised April 2017.

${ }^{1}$ Currently working at Université Côte d'Azur, UMR CNRS 7351, LJAD, France.

${ }^{2}$ Currently working at Technische Universität Berlin, Germany.

MSC2010 subject classifications. Primary 60H15, 60H40; secondary 42B25.

Key words and phrases. Singular SPDEs, paracontrolled calculus. 
1. Introduction. We study in this work the following Cauchy problem:

$$
\left\{\begin{array}{l}
\partial_{t} u=\Delta_{\mathbb{T}^{3}} u-u^{3}+\xi, \\
u(0, x)=u^{0}(x), \quad x \in \mathbb{T}^{3},
\end{array}\right.
$$

where $\xi$ is a space-time white noise such that $\int_{\mathbb{T}^{3}} \xi(\cdot, x) \mathrm{d} x=0$, that is, it is a centered Gaussian space-time distribution with covariance function defined formally by

$$
\mathbb{E}[\xi(s, x) \xi(t, y)]=\delta(t-s) \delta(x-y) .
$$

As we will see in the sequel, the solution $u: \mathbb{R}_{+} \times \mathbb{T}^{3} \rightarrow \mathbb{R}$ is expected to be a Schwartz distribution in space and not a function. This will complicate our understanding of the nonlinear part of the equation. In fact, the most challenging aspect of this work is to define the term $u^{3}$ and to control it in a suitable topological space.

To have a better understanding of the problem, let us start by writing this equation in its mild formulation

$$
u=P_{t} u^{0}-\int_{0}^{t} P_{t-s}\left(u_{s}\right)^{3} \mathrm{~d} s+X_{t},
$$

where $P_{t}=e^{t \Delta}$ is the heat flow and $X_{t}=\int_{0}^{t} P_{t-s} \xi_{s} \mathrm{~d} s$ is the solution of the linear equation

$$
\partial_{t} X_{t}=\Delta_{\mathbb{T}^{3}} X_{t}+\xi, \quad X_{0}=0 .
$$

Moreover, $X$ is a Gaussian process and as we will see below $X \in C([0, T]$; $\mathcal{C}^{-1 / 2-\varepsilon}\left(\mathbb{T}^{3}\right)$ ) for every $\varepsilon>0$ where $\mathcal{C}^{\alpha}=B_{\infty, \infty}^{\alpha}$ stands for the Besov-Hölder space (see Section 2.1 for the definition of this space). The main difficulty of the equation (1.1) originates from the fact that for a fixed time $t$ the spatial regularity of the solution $u(t, x)$ cannot be better than the one of $X_{t}$. If we measure the spatial regularity in the scale of Hölder spaces $\mathcal{C}^{\alpha}$, we should expect that $u(t, x) \in \mathcal{C}^{\alpha}\left(\mathbb{T}^{3}\right)$ for any $\alpha<-1 / 2$ but not better. The term $u^{3}$ in particular is not well defined. An organic approach to give a meaning to the equation would consist in regularizing the noise in $\xi^{\varepsilon}=\xi \star \rho^{\varepsilon}$ where $\rho^{\varepsilon}=\varepsilon^{-3} \rho(\dot{\bar{\varepsilon}})$ is an approximation of the identity and then try to get a uniform bound in $\varepsilon$ on the solution $u^{\varepsilon}$ of the approximate equation

$$
\partial_{t} u^{\varepsilon}=\Delta u^{\varepsilon}-\left(u^{\varepsilon}\right)^{3}+\xi^{\varepsilon} .
$$

At this stage, the main problem to pass the limit in this equation is that the nonlinear term will diverge when $\varepsilon$ goes to zero and a suitable renormalization should be introduced to get a nontrivial limit. We will show precisely that if $u^{\varepsilon}$ is a solution of the following modified equation:

$$
\partial_{t} u^{\varepsilon}=\Delta u^{\varepsilon}-\left(\left(u^{\varepsilon}\right)^{3}-C_{\varepsilon} u^{\varepsilon}\right)+\xi^{\varepsilon},
$$


with $C_{\varepsilon}=\frac{a}{\varepsilon}+b \log (\varepsilon)+c$, for a suitable choice of the constants $a, b, c$, then $u^{\varepsilon}$ converges in the sense of distributions ${ }^{3}$ to a nontrivial random field $u$ which does not depend on the choice of the mollifier $\rho$. Therefore, the aim of this work is to give a meaning to equation (1.2) and obtaining a (local in time) solution.

The method developed in this paper uses some ideas of [9] where the author deals with the KPZ equation. More precisely, we will expand the solution as the sum of stochastic objects involving the Gaussian field $X$ and derive an equation for the remainder term, which can be solved by a fixed point argument using the notion of paracontrolled distributions introduced in [8]. A solution of this equation has already been constructed by M.Hairer in [10] where the author shows the convergence of the solution of the mollified equation (1.5).

The stochastic quantization problem has been studied since the 1980s in theoretical physics (see, e.g., [13] and [14]). Indeed the solution of the equation (1.1) is expected to be a natural reversible dynamic for the $\Phi_{3}^{4}$ measure $v$ appearing in quantum field theory and given formally by

$$
\nu(\mathrm{d} \varphi) \propto \exp \left(-2 \int_{\mathbb{T}^{3}} \frac{1}{2}|\nabla \varphi|^{2}+\frac{1}{4} \varphi^{4}-“ \infty ” \varphi^{2}\right) \mathrm{d} \varphi .
$$

The correct meaning and construction of this measure is highly nontrivial and was done in the beginning of the 1970s [6]. Let us point out that well-posedness results were already obtained in the two-dimensional setting, for weak probabilistic solutions, by Jona-Lasinio and Mitter in [13, 14]. Some other probabilistic results are obtained thanks to nonperturbative methods by Bertini, Jona-Lasinio and Parrinello in [2]. In [4], Da Prato and Debussche found a strong (in the probabilistic sense) formulation for the two-dimensional problem. More recently, the infinite volume setting was also investigated in [16] and a global existence result is proved for the $\Phi_{4}^{2}$ equation on $\mathbb{R}^{2}$.

On the other side, it is conjectured in [3] that the study of the $\Phi^{4}$ model is related to the Ising model under Glauber dynamic near its critical temperature. Recently, this conjecture was made rigorous in the two-dimensional setting by J. C. Mourrat and $\mathrm{H}$. Weber in [15].

In a recent work, M. Hairer [10] solved the three-dimensional case thanks to his theory of regularity structures. It would be wise to observe that the scope of the theory of regularity structures goes beyond the $\Phi_{3}^{4}$ model and can treat a large class of semilinear parabolic stochastic partial differential equations.

In [8], the authors have introduced a different approach to handle singular equations, namely the method of paracontrolled distributions. Even if this notion is less striking and cannot cover at the moment all the local-well posedness results

\footnotetext{
${ }^{3}$ Actually the convergence takes place in a stronger topology; see Corollary 1.5 for the precise result.
} 
obtained via Hairer's approach, it has the advantage to be elementary and more explicit, which can be useful when one wants to tackle the problem of nonexplosion in time (see $[16,17]$ for such matters).

We will split the proof of the convergence of equation (1.5) into two steps. The first step is purely analytic and does not rely on the fact that $\xi$ is a Gaussian white noise. First, we will extend the flow of the following equation:

$$
\partial_{t} u_{t}=\Delta u_{t}-u_{t}^{3}+3 a u_{t}+9 b u_{t}+\xi_{t}, \quad(a, b) \in \mathbb{R}^{2}, \xi \in C\left([0, T], C^{\infty}\left(\mathbb{T}^{3}\right)\right),
$$

to the situation of more irregular driving Schwartz distribution $\xi$. More precisely, we will prove that the solution $u$ is a continuous function of $\left(u^{0}, R_{a, b} X(\xi)\right)$ with

$$
\begin{aligned}
R_{a, b} X(\xi)= & \left(X, X^{2}-a, I\left(X^{3}-3 a X\right), I\left(X^{3}-3 a X\right) \circ X,\right. \\
& \left.I\left(X^{2}-a\right) \circ\left(X^{2}-a\right)-b, I\left(X^{3}-3 a X\right) \circ\left(X^{2}-a\right)-3 b X\right) .
\end{aligned}
$$

Here, $X_{t}=\int_{0}^{t} P_{t-s} \xi \mathrm{d} s, f \circ g$ denotes the part of the product between $f$ and $g$ where the two function have the same frequency (see Proposition 2.3 for the exact definition) and $I(f)_{t}=\int_{0}^{t} P_{t-s} f \mathrm{~d} s$. This extension is given in the following theorem.

TheOREM 1.1. Let $F: \mathcal{C}^{1}\left(\mathbb{T}^{3}\right) \times C\left(\mathbb{R}^{+}, \mathcal{C}^{0}\left(\mathbb{T}^{3}\right)\right) \times \mathbb{R} \times \mathbb{R} \rightarrow C\left(\mathbb{R}^{+}, \mathcal{C}^{1}\left(\mathbb{T}^{3}\right)\right)$ be the flow of the equation

$$
\left\{\begin{array}{l}
\partial_{t} u_{t}=\Delta u_{t}-u_{t}^{3}+3 a u_{t}+9 b u_{t}+\xi_{t}, \quad t \in\left[0, T_{C}\left(u^{0}, X,(a, b)\right)\right), \\
\partial_{t} u_{t}=0, \quad t \geq T_{C}\left(u^{0}, X,(a, b)\right), \\
u(0, x)=u^{0}(x) \in \mathcal{C}^{1}\left(\mathbb{T}^{3}\right),
\end{array}\right.
$$

where $\xi \in C\left(\mathbb{R}^{+}, \mathcal{C}^{0}\left(\mathbb{T}^{3}\right)\right)$ and $T_{C}\left(u^{0}, \xi,(a, b)\right)$ is a time such that the equation holds for $t \leq T_{C}$. Now let $z \in(1 / 2,2 / 3)$, then there exists a Polish space $\mathcal{X}$, called the space of rough distributions, $\tilde{T}_{C}: \mathcal{C}^{-z} \times \mathcal{X} \rightarrow \mathbb{R}^{+}$a lower semicontinuous function and $\tilde{F}: \mathcal{C}^{-z} \times \mathcal{X} \rightarrow C\left(\mathbb{R}^{+}, C^{-z}\left(\mathbb{T}^{3}\right)\right)$ continuous in $\left(u^{0}, \mathbb{X}\right) \in C^{-z}\left(\mathbb{T}^{3}\right) \times$ $\mathcal{X}$ such that $(\tilde{F}, \tilde{T})$ extends $(F, T)$ in the following sense:

$$
T_{C}\left(u^{0}, \xi,(a, b)\right) \geq \tilde{T}_{C}\left(u^{0}, R_{a, b} X(\xi)\right)>0
$$

and

$$
F\left(u^{0}, \xi, a, b\right)(t)=\tilde{F}\left(u^{0}, R_{a, b} X(\xi)\right)(t) \quad \text { for all } t \leq \tilde{T}_{C}\left(u^{0}, R_{a, b} X(\xi)\right),
$$

for all $\left(u^{0}, \xi\right) \in \mathcal{C}^{1}\left(\mathbb{T}^{3}\right) \times C\left(\mathbb{R}^{+}, \mathcal{C}^{0}\left(\mathbb{T}^{3}\right)\right),(a, b) \in \mathbb{R}^{2}$ with $X_{t}=\int_{0}^{t} \mathrm{~d} s P_{t-s} \xi$ and where $R_{a, b}^{\varphi}$ is given in (1.7).

REMARK 1.2. Let us remark that the vector appearing in the right-hand side of (1.7) does not depend on $\xi$ in the sense that it can be defined for every function $X$ in $C\left([0, T], C^{\infty}\left(\mathbb{T}^{2}\right)\right)$. In that case, we will keep simply the same notation $R_{a, b} X$ for it. 
In a second part, we obtain probabilistic estimates for the stationary OrnsteinUhlenbeck (O.U.) process which is the solution of the linear equation (1.3) and this allows us to construct the rough distribution in that case.

THEOREM 1.3. Let $X$ be the stationary O.U. process and $X^{\varepsilon}$ be a spatial mollification of $X$ defined by

$$
X_{t}^{\varepsilon}=\sum_{k \in \mathbb{Z}^{3}} f(\varepsilon k) \hat{X}_{t}(k) e_{k}, \quad t \geq 0,
$$

where $\hat{X}$ is the Fourier transform of $X$ in the space variable, $\left(e_{k}\right)_{k \in \mathbb{Z}^{3}}$ the Fourier basis of $L^{2}\left(\mathbb{T}^{3}\right)$ and $f$ is a smooth function with compact support which satisfies $f(0)=1$. Then there exists two diverging constants (not unique) $C_{1}^{\varepsilon}, C_{2}^{\varepsilon} \rightarrow^{\varepsilon \rightarrow 0}$ $+\infty$ such that $R_{C_{1}^{\varepsilon}, C_{2}^{\varepsilon}} X^{\varepsilon}$ converges in $L^{p}(\Omega, \mathcal{X})$ for all $p>1$. Moreover, the limit $\mathbb{X} \in \mathcal{X}$ does not depend on the choice of the mollification $f$ and the first component of $\mathbb{X}$ is $X$.

REMARK 1.4. The choice of the constants $C_{1}^{\varepsilon}, C_{2}^{\varepsilon}$ is not unique and depends in general on the choice of the mollification $f$. However, as is mentioned in [11], the constant $C_{2}^{\varepsilon}$ can be taken independently of the mollification.

In this setting, the corollary below follows immediately.

COROLlaRy 1.5. Let $\xi$ be a space time white noise, and $\xi^{\varepsilon}$ be a spatial mollification of $\xi$ such that

$$
\xi^{\varepsilon}=\sum_{k \neq 0} f(\varepsilon k) \hat{\xi}(k) e_{k},
$$

where we have adopted the same assumptions and notation as in Theorem 1.3. Let $X$ be the stationary O.U. process associated to $\xi, \mathbb{X}$ the element of $\mathcal{X}$ given by Theorem 1.3 and $u^{0} \in \mathcal{C}^{-z}$ for $z \in(1 / 2,2 / 3)$. Then there exists a sequence of positive time $T^{\varepsilon}$ which converges almost surely to a random time $T>0$ and such that the solution $u^{\varepsilon}$ of the mollified equation

$$
\left\{\begin{array}{l}
\partial_{t} u_{t}^{\varepsilon}=\Delta u_{t}^{\varepsilon}-\left(u_{t}^{\varepsilon}\right)^{3}+3 C_{1}^{\varepsilon} u_{t}+9 C_{2}^{\varepsilon} u_{t}+\xi_{t}^{\varepsilon}, \quad t \in\left[0, T^{\varepsilon}\right), \\
\partial_{t} u_{t}^{\varepsilon}=0, \quad t \geq T^{\varepsilon} \\
u(0, x)=\left(u^{0}\right)^{\varepsilon}(x)
\end{array}\right.
$$

converges to $\tilde{F}\left(u^{0}, \mathbb{X}\right)$. Here, the limit is understood in the probability sense in the space $C\left(\mathbb{R}^{+}, \mathcal{C}^{-z}\right)$. 
Plan of the paper. The aim of Section 2 is to introduce the basic analytic tools, and to give an extensive heuristic description of the strategy of the proof. In Section 3.3, we introduced the space of paracontrolled distributions, and we prove that for a small time the application associated to the renormalized equation is a contraction, which by a fixed-point argument, gives the existence and uniqueness of the solution, but also the continuity with respect to the rough distribution and the initial condition. Section 4 is devoted to the existence of the rough distribution for the O.U. process.

2. Basic tools, description of the proof and rough distributions. This section is divided into two parts. In the first one, we gives the basic analytical tools needed to fulfill the program of this article. In a second part, we intensively described the strategy of the proof, by pointing out the use of the different propositions of the first subsection.

2.1. Besov spaces and paradifferential calculus. The results given in this subsection can be found in [1] and [8]. Let us start by recalling the definition of Besov spaces via the Littelwood-Paley projectors.

Let $\chi, \theta \in \mathcal{D}$ be a nonnegative radial functions such that:

1. The support of $\chi$ is contained in a ball and the support of $\theta$ is contained in an annulus;

2. $\chi(\xi)+\sum_{j \geq 0} \theta\left(2^{-j} \xi\right)=1$ for all $\xi \in \mathbb{R}^{d}$;

3. $\operatorname{supp}(\chi) \cap \operatorname{supp}\left(\theta\left(2^{-j} \cdot\right)\right)=\varnothing$ for $i \geq 1$ and $\operatorname{supp}\left(\theta\left(2^{-j} \cdot\right)\right) \cap \operatorname{supp}\left(\theta\left(2^{-i} \cdot\right)\right)=$ $\varnothing$ when $|i-j|>1$.

For the existence of $\chi$ and $\theta$, see Proposition 2.10 in [1]. The Littlewood-Paley blocks are defined as

$$
\Delta_{-1} u=\mathscr{F}^{-1}(\chi \mathscr{F} u) \quad \text { and } \quad \text { for } j \geq 0, \quad \Delta_{j} u=\mathscr{F}^{-1}\left(\theta\left(2^{-j} \cdot\right) \mathscr{F} u\right),
$$

where $\mathscr{F}$ denotes the Fourier transform. We define the Besov space of distribution by

$$
B_{p, q}^{\alpha}=\left\{u \in S^{\prime}\left(\mathbb{R}^{d}\right) ;\|u\|_{B_{p, q}^{\alpha}}^{q}=\sum_{j \geq-1} 2^{j q \alpha}\left\|\Delta_{j} u\right\|_{L^{p}}^{q}<+\infty\right\} .
$$

In the sequel, we will deal with the special case of $\mathcal{C}^{\alpha}:=B_{\infty, \infty}^{\alpha}$ and write $\|u\|_{\alpha}=$ $\|u\|_{B_{\infty, \infty}^{\alpha}}$. We give the following result for the convergence of localized series in Besov spaces, which will prove itself useful.

Proposition 2.1. Let $(p, q, s) \in[1,+\infty]^{2} \times \mathbb{R}, B$ be a ball in $\mathbb{R}^{d}$ and $\left(u_{j}\right)_{j \geq-1}$ be a sequence of functions such that $\operatorname{supp}\left(u_{j}\right)$ is contained in $2^{j}$ B. Moreover, we assume that

$$
\Xi_{p, q, s}=\left\|\left(2^{j s}\left\|u_{j}\right\|_{L^{p}}\right)_{j \geq-1}\right\|_{l q}<+\infty .
$$

Then $u=\sum_{j \geq-1} u_{j} \in B_{p, q}^{s}$ and $\|u\|_{B_{p, q}^{s}} \lesssim \Xi_{p, q, s}$. 
The trick to manipulate stochastic objects is to deal with Besov spaces with finite integrability exponent and then to go back to the space $\mathcal{C}^{\alpha}$. For that, we will use the following embedding result.

Proposition 2.2. Let $1 \leq p_{1} \leq p_{2} \leq+\infty$ and $1 \leq q_{1} \leq q_{2} \leq+\infty$. For all $s \in \mathbb{R}$, the space $B_{p_{1}, q_{1}}^{s}$ is continuously embedded in $B_{p_{2}, q_{2}}^{s-d\left(\frac{1}{p_{1}}-\frac{1}{p_{2}}\right)}$, in particular we have $\|u\|_{\alpha-\frac{d}{p}} \lesssim\|u\|_{B_{p, p}^{\alpha}}$.

Taking $f \in \mathcal{C}^{\alpha}$ and $g \in \mathcal{C}^{\beta}$, we can formally decompose the product as

$$
f g=f \prec g+f \circ g+f \succ g,
$$

where

$$
f \prec g=g \succ f=\sum_{j \geq-1} \sum_{i<j-1} \Delta_{i} f \Delta_{j} g \quad \text { and } \quad f \circ g=\sum_{j \geq-1} \sum_{|i-j| \leq 1} \Delta_{i} f \Delta_{j} g .
$$

With this notation, the following results hold.

Proposition 2.3 (Bony estimates). Let $\alpha, \beta \in \mathbb{R}$ :

- $\|f \prec g\|_{\beta} \lesssim\|f\|_{\infty}\|g\|_{\beta}$ for $f \in L^{\infty}$ and $g \in \mathcal{C}^{\beta}$.

- $\|f \succ g\|_{\alpha+\beta} \lesssim\|f\|_{\alpha}\|g\|_{\beta}$ for $\beta<0, f \in \mathcal{C}^{\alpha}$ and $g \in \mathcal{C}^{\beta}$.

- $\|f \circ g\|_{\alpha+\beta} \lesssim\|f\|_{\alpha}\|g\|_{\beta}$ for $\alpha+\beta>0$ and $f \in \mathcal{C}^{\alpha}$ and $g \in \mathcal{C}^{\beta}$.

A simple consequence of these estimates is that the product $f g$ between two Besov distributions $f \in \mathcal{C}^{\alpha}$ and $g \in \mathcal{C}^{\beta}$ is well defined if $\alpha+\beta>0$ Moreover, it satisfies $\|f g\|_{\min (\alpha, \beta)} \lesssim\|f\|_{\alpha}\|g\|_{\beta}$.

One of the key results of [8] is a commutation lemma for the operator $\prec$ and $\circ$.

Proposition 2.4 (Commutator estimate). Let $\alpha, \beta, \gamma \in \mathbb{R}$ be such that $\alpha<$ $1, \alpha+\beta+\gamma>0$ and $\beta+\gamma<0$ then

$$
R(f, x, y)=(f \prec x) \circ y-f(x \circ y)
$$

is well defined for $f \in \mathcal{C}^{\alpha}, x \in \mathcal{C}^{\beta}$ and $y \in \mathcal{C}^{\gamma}$. More precisely,

$$
\|R(f, x, y)\|_{\alpha+\beta+\gamma} \lesssim\|f\|_{\alpha}\|x\|_{\beta}\|y\|_{\gamma} .
$$

We finish this section by describing the action of the heat flow on Besov spaces and by giving a commutation property with the paraproduct. See the Appendix for a proof. 
LEMMA 2.5 (Heat-flow estimates). Let $\theta \geq 0$ and $\alpha \in \mathbb{R}$. The following inequalities:

$$
\left\|P_{t} f\right\|_{\alpha+2 \theta} \lesssim \frac{1}{t^{\theta}}\|f\|_{\alpha}, \quad\left\|\left(P_{t-s}-1\right) f\right\|_{\alpha-2 \varepsilon} \lesssim|t-s|^{\varepsilon}\|f\|_{\alpha},
$$

holds for all $f \in \mathcal{C}^{\alpha}$. Moreover, if $\alpha<1$ and $\beta \in \mathbb{R}$ we have

$$
\left\|P_{t}(f \prec g)-f \prec P_{t} g\right\|_{\alpha+\beta+2 \theta} \lesssim \frac{1}{t^{\theta}}\|f\|_{\alpha}\|g\|_{\beta},
$$

for all $g \in \mathcal{C}^{\beta}$.

Let us now introduce some notations for functional spaces which will be used extensively in the sequel of this paper.

NOTATION 2.6.

$$
C_{T}^{\beta}=C\left([0, T] ; \mathcal{C}^{\beta}\right)
$$

For $f \in C_{T}^{\beta}$, we introduce the norm

$$
\|f\|_{\beta}=\sup _{t \in[0, T]}\left\|f_{t}\right\|_{\mathcal{C}^{\beta}}=\sup _{t \in[0, T]}\left\|f_{t}\right\|_{\beta}
$$

and the space

$$
C_{T}^{\alpha, \beta}:=C^{\alpha}\left([0, T], \mathcal{C}^{\beta}\left(\mathbb{T}^{3}\right)\right) .
$$

We denote the space of $\alpha$-Hölder functions in time with value in the Besov space $\mathcal{C}^{\beta}$, where $\alpha>0$. Furthermore, we endow this space with the following distance:

$$
d_{\alpha, \beta}(f, g)=\sup _{t \neq s \in[0, T]} \frac{\left\|(f-g)_{t}-(f-g)_{s}\right\|_{\beta}}{|t-s|^{\alpha}}+\sup _{t \in[0, T]}\left\|f_{t}-g_{t}\right\|_{\beta} .
$$

Let us now end this section by giving a proposition which is a consequence of Lemma 2.5, in which we describe the action of the operator $I$ on the Besov spaces.

Proposition 2.7 (Schauder estimates). Let $\beta \in \mathbb{R}, f \in C_{T}^{\beta}$ and $I(f)(t)=$ $-\int_{0}^{t} P_{t-s} f_{s} \mathrm{~d} s$, then the following bound holds fo all $\theta<1$ :

$$
\|I(f)\|_{C_{T}^{\beta+2 \theta}} \lesssim T^{1-\theta}\|f\|_{C_{T}^{\beta}}
$$

Moreover, if $\alpha \in(0,1), \beta>0, f \in C_{T}^{\alpha, \beta}$ and $g \in C_{T}^{\gamma}$ then the following commutation estimate holds:

$$
\|I(f \prec g)-f \prec I(g)\|_{C_{T}^{\gamma+2 \theta}} \lesssim T^{\kappa}\|f\|_{C_{T}^{\alpha, \beta}}\|g\|_{C_{T}^{\gamma}},
$$

for all $\theta<\min (\alpha, \beta)+1$ with $\kappa=\min (1-\theta+\beta / 2,1-\theta+\gamma)$. 
ProOF. Only the second estimate requires a proof since the first one is an immediate consequence of the heat-flow estimates. To get the second bound, let us observe that

$$
I(f \prec g)(t)-f \prec I(g)(t)=I_{1}(t)+I_{2}(t),
$$

where

$$
\begin{aligned}
& I_{1}(t)=\int_{0}^{t}\left(f_{s} \prec P_{t-s} g_{s}\right)-P_{t-s}\left(f_{s} \prec g_{s}\right) \mathrm{d} s \quad \text { and } \\
& I_{2}(t)=\int_{0}^{t}\left(f_{t}-f_{s}\right) \prec P_{t-s} g_{s} \mathrm{~d} s .
\end{aligned}
$$

Now using the heat-flow estimate we have that

$$
\begin{aligned}
\left\|I_{1}(t)\right\|_{\mathcal{C}^{\gamma+\beta+2 \theta^{\prime}}} & \lesssim \int_{0}^{t}\left\|\left(f_{s} \prec P_{t-s} g_{s}\right)-P_{t-s}\left(f_{s} \prec g_{s}\right)\right\|_{\mathcal{C}^{\beta+\gamma+2 \theta^{\prime}}} \mathrm{d} s \\
& \lesssim\left(\int_{0}^{t}(t-s)^{-\theta^{\prime}} \mathrm{d} s\right)\|f\|_{C_{T}^{\alpha, \beta}}\|g\|_{C_{T}^{\gamma}},
\end{aligned}
$$

for all $\theta^{\prime}<1$. The second inequality is obtained by using the heat-flow estimates. Taking $2 \theta=2 \theta^{\prime}+\beta$ gives the needed bound for $I_{1}$. The bound of $I_{2}$ is a consequence of the the Bony estimate for the paraproduct term, the Hölder regularity in time of $f$ and the heat flow estimate. Indeed, we have that

$$
\left\|I_{2}(t)\right\|_{\mathcal{C}^{\gamma+2 \theta}} \lesssim \int_{0}^{t}\left\|\left(f_{t}-f_{S}\right)\right\|_{\mathcal{C}^{\beta}}\left\|P_{t-s} g_{s}\right\|_{\mathcal{C}^{\gamma+2 \theta}} \mathrm{d} s \lesssim T^{1-(\theta-\gamma)}\|f\|_{C_{T}^{\alpha, \beta}}\|g\|_{C_{T}^{\gamma}}
$$

which completes the proof.

2.2. Description of the strategy and renormalized equation. Let us focus on the mild formulation of equation (1.1)

$$
u=\Psi+X+I\left(u^{3}\right)=X+\Phi,
$$

where we recall the notation $I(f)(t)=-\int_{0}^{t} P_{t-s} f_{s} \mathrm{~d} s, X=-I(\xi)$ and $\Psi_{t}=P_{t} u^{0}$ for $u^{0} \in \mathcal{C}^{-z}\left(\mathbb{T}^{3}\right)$. We can see that a solution $u$ must have the same regularity as $X$. However, it is well known that for all $\varepsilon>0$ we have $X \in C\left([0, T], \mathcal{C}^{-1 / 2-\varepsilon}\right)$. (See Section 4 for a quick proof of this.) But in that case the nonlinear term $u^{3}$ is not well defined, as there is no universal notion for the product of distributions. A first idea is to proceed by regularization of $X$, such that products of the regularized quantities are well defined, and then try to pass to the limit. Let us recall that the stationary O.U process $\left(\hat{X}_{t}(k)\right)_{t \in \mathbb{R}, k \in \mathbb{Z}^{3}}$ is a centered Gaussian process with covariance function given by

$$
\mathbb{E}\left[\hat{X}_{t}(k) \hat{X}_{s}\left(k^{\prime}\right)\right]=\delta_{k+k^{\prime}=0} \frac{e^{-|k|^{2}|t-s|}}{|k|^{2}}
$$


and $\hat{X}_{t}(0)=0$. Let $X_{t}^{\varepsilon}$ be the mollification of $X$ introduced in Theorem 1.3. Then the following approximated equation:

$$
\Phi^{\varepsilon}=\Psi^{\varepsilon}+I\left(\left(X^{\varepsilon}\right)^{3}\right)+3 I\left(\left(\Phi^{\varepsilon}\right)^{2} X^{\varepsilon}\right)+3 I\left(\Phi^{\varepsilon}\left(X^{\varepsilon}\right)^{2}\right)+I\left(\left(\Phi^{\varepsilon}\right)^{3}\right),
$$

where $\Phi^{\varepsilon}=I\left(\left(u^{\varepsilon}\right)^{3}\right)+\Psi^{\varepsilon}$ is well-posed. Before proceeding into the analysis of this equation, let us observe that a straightforward computation gives

$$
\begin{aligned}
C_{1}^{\varepsilon}:=\mathbb{E}\left[\left(X_{t}^{\varepsilon}\right)^{2}\right] & =\sum_{k \in \mathbb{Z}^{3}-\{0\}} \sum_{k_{1}+k_{2}=k} f\left(\varepsilon k_{1}\right) f\left(\varepsilon k_{2}\right) \frac{1}{\left|k_{1}\right|^{2}} \delta_{k_{1}+k_{2}=0} \\
& =\sum_{k \in \mathbb{Z}^{3}-\{0\}} \frac{|f(\varepsilon k)|^{2}}{|k|^{2}} \sim 0 \frac{1}{\varepsilon} \int_{\mathbb{R}} f(x)|x|^{-2} \mathrm{~d} x .
\end{aligned}
$$

Here, $A_{\varepsilon} \sim_{0} B_{\varepsilon}$ means that when $\varepsilon$ is close to 0 , the quantity $A_{\varepsilon}$ can be be bounded from below and above by a positive constant times $B_{\varepsilon}$. Then there is no hope of obtaining a finite limit for $\left(X^{\varepsilon}\right)^{2}$ when $\varepsilon$ goes to zero. This difficulty has to be solved by subtracting from the original equation these problematic contributions. In order to do so consistently, we will introduce a renormalized product. Formally, we would like to define

$$
X^{\diamond 2}=X^{2}-\mathbb{E}\left[X^{2}\right]
$$

and show that it is well defined and that $X^{\diamond 2} \in C_{T}^{-1-\delta}$ for $\delta>0$. Precisely, we will introduce

$$
\left(X^{\varepsilon}\right)^{\diamond 2}=\left(X^{\varepsilon}\right)^{2}-\underbrace{\mathbb{E}\left[\left(X^{\varepsilon}\right)^{2}\right]}_{=: C_{1}^{\varepsilon}}
$$

and we will prove that it converges to some finite limit. It would be wise to remark that many other terms need to be renormalized and subtracting the constant $C_{1}^{\varepsilon}$ is not enough to take care of them. Indeed, as we will see in Section 4, a second renormalization constant $C_{2}^{\varepsilon}$ is needed. Including such considerations in the mollified equation gives rise to an algebraic renormalization term which takes the form $-C^{\varepsilon} I\left(\Phi^{\varepsilon}+X^{\varepsilon}\right)$ with $C^{\varepsilon}=3\left(C_{1}^{\varepsilon}-3 C_{2}^{\varepsilon}\right)$. More precisely, we need to study the following equation:

$$
\begin{aligned}
\Phi^{\varepsilon}= & \Psi^{\varepsilon}+I\left(\left(X^{\varepsilon}\right)^{3}\right)+3 I\left(\left(\Phi^{\varepsilon}\right)^{2} X^{\varepsilon}\right)+3 I\left(\Phi^{\varepsilon}\left(X^{\varepsilon}\right)^{2}\right) \\
& +I\left(\left(\Phi^{\varepsilon}\right)^{3}\right)-C^{\varepsilon} I\left(\Phi^{\varepsilon}+X^{\varepsilon}\right) \\
= & \Psi^{\varepsilon}+I\left(\left(X^{\varepsilon}\right)^{3}-3 C_{1}^{\varepsilon} X^{\varepsilon}\right)+3 I\left(\left(\Phi^{\varepsilon}\right)^{2} X^{\varepsilon}\right) \\
& +3 I\left(\Phi^{\varepsilon}\left(\left(X^{\varepsilon}\right)^{2}-C_{1}^{\varepsilon}\right)+9 C_{2}^{\varepsilon}\left(\Phi^{\varepsilon}+X^{\varepsilon}\right)\right)+I\left(\left(\Phi^{\varepsilon}\right)^{3}\right),
\end{aligned}
$$

or in an other form

$$
\Phi^{\varepsilon}=\Psi^{\varepsilon}+I\left(\left(X^{\varepsilon}\right)^{\diamond 3}\right)+3 I\left(\left(\Phi^{\varepsilon}\right)^{2} X^{\varepsilon}\right)+3 I\left(\Phi^{\varepsilon} \diamond\left(X^{\varepsilon}\right)^{\diamond 2}\right)+I\left(\left(\Phi^{\varepsilon}\right)^{3}\right) .
$$


We have adopted the following notation:

$$
\begin{aligned}
I\left(\Phi^{\varepsilon} \diamond\left(X^{\varepsilon}\right)^{\diamond 2}\right) & :=3 I\left(\Phi^{\varepsilon}\left(\left(X^{\varepsilon}\right)^{\diamond 2}\right)\right)+9 C_{2}^{\varepsilon} I\left(\Phi^{\varepsilon}+X^{\varepsilon}\right), \\
I\left(\left(X^{\varepsilon}\right)^{\diamond 3}\right) & =I\left(\left(X^{\varepsilon}\right)^{3}-3 C_{1}^{\varepsilon} X^{\varepsilon}\right) .
\end{aligned}
$$

A brief analysis of the wanted regularity for the involved objects shows that even if the terms $I\left(\left(X^{\varepsilon}\right)^{\diamond 2}\right)$ and $I\left(\left(X^{\varepsilon}\right)^{\diamond 3}\right)$ converge in the suitable spaces, the renormalization introduced before is not enough to define the equation. Indeed, from Section 4 one can see that for all $\delta>0, X^{\varepsilon}$ converges in probability (and even almost surely) in the space $C_{T}^{-1 / 2-\delta},\left(X^{\varepsilon}\right)^{\diamond 2}$ converges in the space $\mathcal{C}_{T}^{-1-\delta}$ and the term $I\left(\left(X^{\varepsilon}\right)^{\diamond 3}\right)$ converges in the space $C_{T}^{1 / 2-\delta}$. So we can expect that the presumed limit $\Phi$ of the solution $\Phi^{\varepsilon}$ has the same regularity as the worst term in the last equation. Hence, $\Phi \in C_{T}^{1 / 2-\delta}$ and the estimates of Proposition 2.3 are not enough to take care of the terms $X^{\diamond 2} \Phi$ and $X \Phi^{2}$, since the sums of the regularities are still negative. Nevertheless, it is expected from Section 4 that if those terms are constructed they lie respectively in $C_{T}^{-1-\delta}$ and $C_{T}^{-1 / 2-\delta}$. One expects from Section 4 that the solution $\Phi^{\varepsilon}$ may converge as soon as it is expressed as functional of "purely stochastic" terms. In order to have such a decomposition, we will use extensively the definition of the paraproduct and the commutator estimates. Proposition 2.3 allows us to deal with products of factors as soon as the sum of the spatial regularity is positive. The commutator and Schauder estimates (Propositions 2.4 and 2.7) allow us to decompose the analytically ill-defined terms into purely stochastic factors and well-defined terms.

For the sake of better comprehension, we will consider only the null initial condition. It does not change the algebraic part which is exposed here, but pushes us to deal with space of continuous functions for strictly positive time which can blowup at the origin. The equation becomes

$$
\begin{aligned}
\Phi^{\varepsilon}= & \underbrace{I\left(\left(X^{\varepsilon}\right)^{\diamond 3}\right)}_{C_{T}^{1 / 2-\delta}}+\underbrace{3 I\left(\left(X^{\varepsilon}\right)^{\diamond 2} \Phi^{\varepsilon}\right)}_{C_{T}^{1-\delta}}+\underbrace{9 C_{2}^{\varepsilon} I\left(X^{\varepsilon}+\Phi^{\varepsilon}\right)}_{C_{T}^{3 / 2-\delta}} \\
& +\underbrace{3 I\left(X^{\varepsilon}\left(\Phi^{\varepsilon}\right)^{2}\right)}_{C_{T}^{3 / 2-\delta}}+\underbrace{I\left(\left(\Phi^{\varepsilon}\right)^{3}\right)}_{C_{T}^{3 / 2-\delta}} .
\end{aligned}
$$

The form of the equation suggests to make the following ansatz about the a priori expression of the solution $\Phi^{\varepsilon}$.

AnSATZ 2.8. We suppose that there exists $\left(\Phi^{\varepsilon}\right)^{b}$ such that $\Phi^{\varepsilon}=I\left(\left(X^{\varepsilon}\right)^{\diamond 3}\right)+$ $\left(\Phi^{\varepsilon}\right)^{b}$ and where for all $\delta>0$ (small enough) the reminder $\left(\Phi^{\varepsilon}\right)^{b}$ is uniformly bounded (in $\varepsilon$ ) in the space $C_{T}^{1-\delta}$. 
When $\Phi^{\varepsilon}$ fulfills Ansatz 2.8, one can develop the fourth term of the right-hand side and obtain

$$
X^{\varepsilon}\left(\Phi^{\varepsilon}\right)^{2}=2 X^{\varepsilon}\left(\Phi^{\varepsilon} \prec \Phi^{\varepsilon}\right)+X^{\varepsilon}\left(\Phi^{\varepsilon} \circ \Phi^{\varepsilon}\right) .
$$

As $\Phi^{\varepsilon} \in C_{T}^{1 / 2-\delta}$ and $X^{\varepsilon} \in C_{T}^{-1 / 2-\delta}$, the term $X^{\varepsilon}\left(\Phi^{\varepsilon} \circ \Phi^{\varepsilon}\right)$ is well defined thanks to Proposition 2.3. It is possible to develop the first one a bit further in order to have

$$
\begin{aligned}
X^{\varepsilon}\left(\Phi^{\varepsilon} \prec \Phi^{\varepsilon}\right)= & X^{\varepsilon} \circ\left(\Phi^{\varepsilon} \prec \Phi^{\varepsilon}\right) \\
& +\left(X^{\varepsilon} \prec\left(\Phi^{\varepsilon} \prec \Phi^{\varepsilon}\right)+\left(\Phi^{\varepsilon} \prec \Phi^{\varepsilon}\right) \prec X^{\varepsilon}\right) .
\end{aligned}
$$

Here again, the only ill-defined term may be the first one. Hopefully, the regularities of the objects allow us to use the commutator estimate of Proposition 2.4. We then use the Ansatz 2.8 once again to get

$$
\begin{aligned}
X^{\varepsilon} \circ\left(\Phi^{\varepsilon} \prec \Phi^{\varepsilon}\right) & =\Phi^{\varepsilon}\left(\Phi^{\varepsilon} \circ X^{\varepsilon}\right)+R\left(X^{\varepsilon}, \Phi^{\varepsilon}, \Phi^{\varepsilon}\right) \\
& =\Phi^{\varepsilon}\left(I\left(X^{\varepsilon}\right)^{3} \circ X^{\varepsilon}+\left(\Phi^{\varepsilon}\right)^{b} \circ X^{\varepsilon}\right)+R\left(X^{\varepsilon}, \Phi^{\varepsilon}, \Phi^{\varepsilon}\right) .
\end{aligned}
$$

Hence, Ansatz 2.8 allows us to see the product $\left(\Phi^{\varepsilon}\right)^{2} X^{\varepsilon}$ as a continuous functional of $\left(\Phi^{\varepsilon}\right)^{b}$ and some stochastic well-defined terms. This fact is summarized in the following proposition.

Proposition 2.9. Let $\Phi^{\varepsilon}$ be as in Ansatz 2.8 then $I\left(\left(\Phi^{\varepsilon}\right)^{2} X^{\varepsilon}\right)$ is a continuous functional (bounded uniformly in $\varepsilon$ ) of $\left(\Phi^{\varepsilon}\right)^{b}, X^{\varepsilon}, I\left(\left(X^{\varepsilon}\right)^{\diamond 3}\right),\left(X^{\varepsilon}\right)^{\diamond 2}$ and $I\left(\left(X^{\varepsilon}\right)^{\diamond 3}\right) \circ X^{\varepsilon}$. Moreover, if each of these data has a finite limit in the prescribed space then $I\left(\left(\Phi^{\varepsilon}\right)^{2} X^{\varepsilon}\right)$ is also convergent.

The aim of Section 3.1, and in particular Proposition 3.6 is to specify the dependencies toward the norm of each object. Furthermore, thanks to Section 4 the term $I\left(\left(X^{\varepsilon}\right)^{\diamond 3}\right) \circ X^{\varepsilon}$ converges in probability in the suitable space.

Notation 2.10. Since the eventual limit of $\left(\Phi^{\varepsilon}\right)^{2} X^{\varepsilon}$ is not simply a continuous functional of $X$ (but also of the eventual limit of the stochastic terms appearing in Proposition 2.9) we denote the limiting object by $\Phi^{2} \diamond X$ instead of $\Phi^{2} X$ to keep this fact in mind.

Unfortunately, Ansatz 2.8 is not enough to handle the product $\Phi^{\varepsilon}\left(X^{\varepsilon}\right)^{\diamond 2}$. Indeed, $\left(X^{\varepsilon}\right)^{\diamond 2} \in C_{T}^{-1-\delta}$ and the reminder $\left(\Phi^{\varepsilon}\right)^{b} \in C_{T}^{1-\delta}$. Hence, one has to develop equation (2.3) a bit further. We still assume that $\Phi^{\varepsilon}$ complies with Ansatz 2.8. 
From the paraproduct decomposition, we can see that

$$
\begin{aligned}
\Phi^{\varepsilon}= & \underbrace{I\left(\left(X^{\varepsilon}\right)^{\diamond 3}\right)}_{C_{T}^{1 / 2-\delta}}+\underbrace{3 I\left(\Phi^{\varepsilon} \prec\left(X^{\varepsilon}\right)^{\diamond 2}\right)}_{C_{T}^{1-\delta}} \\
& +\underbrace{3 I\left(\Phi^{\varepsilon} \circ\left(X^{\varepsilon}\right)^{\diamond 2}\right)}_{C_{T}^{3 / 2-\delta}}-\underbrace{9 C_{2}^{\varepsilon} I\left(\Phi^{\varepsilon}+X^{\varepsilon}\right)}_{C^{3 / 2-\delta}} \\
& +\underbrace{3 I\left(\left(X^{\varepsilon}\right)^{\diamond 2} \succ \Phi^{\varepsilon}\right)}_{C_{T}^{3 / 2-\delta}}+\underbrace{3 I\left(X^{\varepsilon}\left(\Phi^{\varepsilon}\right)^{2}\right)}_{C_{T}^{3 / 2-\delta}}+\underbrace{I\left(\left(\Phi^{\varepsilon}\right)^{3}\right)}_{C_{T}^{3 / 2-\delta}} .
\end{aligned}
$$

Let us observe that the only "ill-defined" term in this expansion is $I\left(\Phi^{\varepsilon} \circ\left(X^{\varepsilon}\right)^{\diamond 2}\right)$. Nevertheless, one can make a second stronger ansatz about the representation of the solutions in terms of functions with increasing regularity.

AnsATZ 2.11 (Paracontrol ansatz). We suppose that there exists $\left(\Phi^{\varepsilon}\right)^{\prime}$ such that

$$
\Phi^{\varepsilon}=I\left(\left(X^{\varepsilon}\right)^{\diamond 3}\right)+3 I\left(\left(\Phi^{\varepsilon}\right)^{\prime} \prec\left(X^{\varepsilon}\right)^{\diamond 2}\right)+\left(\Phi^{\varepsilon}\right)^{\sharp}
$$

where for all $\delta>0$ and all $v>0$ small enough $\left(\Phi^{\varepsilon}\right)^{\prime} \in C_{T}^{1 / 2-\delta}$ and $\left(\Phi^{\varepsilon}\right)^{\sharp} \in C_{T}^{1+v}$ uniformly in $\varepsilon$. Moreover, as we will see in the sequel some Hölder regularity in time is also needed for the term $\left(\Phi^{\varepsilon}\right)^{\prime}$ and actually we will assume that this term is uniformly bounded (in $\varepsilon$ ) in the space $C_{T}^{\delta^{\prime}, 1 / 2-\delta}$ for $\delta^{\prime}<\delta / 2$

This ansatz is an informal form of the definition of the paracontrolled distributions (Definition 3.3). It will allow us to prove an analog of Proposition 2.9 but for $I\left(\Phi^{\varepsilon} \circ\left(X^{\varepsilon}\right)^{\diamond 2}\right)$. First, let us remind that $I(f)=-\int_{0}^{t} P_{t-s} f_{s} \mathrm{~d} s$. Thanks to the commutator estimate of Proposition 2.4, the Hölder regularity in time of $\left(\Phi^{\varepsilon}\right)^{\prime}$ and the Schauder estimate Proposition 2.7 we have that for all $v>0$ small enough

$$
I\left(\left(\Phi^{\varepsilon}\right)^{\prime} \prec\left(X^{\varepsilon}\right)^{\diamond 2}\right)-\left(\Phi^{\varepsilon}\right)^{\prime} \prec I\left(X^{\varepsilon}\right)^{\diamond 2} \in \mathscr{C}_{T}^{1+v} .
$$

Moreover, this quantity is continuous with respect to $\left(\Phi^{\varepsilon}\right)^{\prime}$ and $\left(X^{\varepsilon}\right)^{\diamond 2}$. Hence, one can reformulate Ansatz 2.11 in the following way. For all $\delta, v>0$ small enough, there exists $\left(\Phi^{\varepsilon}\right)^{\prime} \in \mathscr{C}_{T}^{1 / 2-\delta}$ and $\left(\Phi^{\varepsilon}\right)^{\natural}$ such that

$$
\Phi^{\varepsilon}=I\left(\left(X^{\varepsilon}\right)^{\diamond 3}\right)+3\left(\Phi^{\varepsilon}\right)^{\prime} \prec I\left(\left(X^{\varepsilon}\right)^{\diamond 2}\right)+\left(\Phi^{\varepsilon}\right)^{\natural} .
$$

Again, the only ill-defined term in $\Phi^{\varepsilon}\left(X^{\varepsilon}\right)^{\diamond 2}$ is the resonant term $\Phi^{\varepsilon} \circ\left(X^{\varepsilon}\right)^{\diamond 2}$. Using the reformulation (2.5) of Ansatz 2.11, we get easily that

$$
\begin{aligned}
\left(\Phi^{\varepsilon}\right) \circ\left(X^{\varepsilon}\right)^{\diamond 2}= & I\left(\left(X^{\varepsilon}\right)^{\diamond 3}\right) \circ\left(X^{\varepsilon}\right)^{\diamond 2}+3\left(X^{\varepsilon}\right)^{\diamond 2} \circ\left(\left(\Phi^{\varepsilon}\right)^{\prime} \prec I\left(\left(X^{\varepsilon}\right)^{\diamond 2}\right)\right) \\
& +\left(\Phi^{\varepsilon}\right)^{\natural} \circ\left(X^{\varepsilon}\right)^{\diamond 2}
\end{aligned}
$$




$$
\begin{aligned}
= & I\left(\left(X^{\varepsilon}\right)^{\diamond 3}\right) \circ\left(X^{\varepsilon}\right)^{\diamond 2}+\left(\Phi^{\varepsilon}\right)^{\prime}\left(I\left(\left(X^{\varepsilon}\right)^{\diamond 2}\right) \circ\left(X^{\varepsilon}\right)^{\diamond 2}\right) \\
& +R\left(\left(X^{\varepsilon}\right)^{\diamond 2},\left(\Phi^{\varepsilon}\right)^{\prime}, I\left(\left(X^{\varepsilon}\right)^{\diamond 2}\right)\right)+\left(\Phi^{\varepsilon}\right)^{\natural} \circ\left(X^{\varepsilon}\right)^{\diamond 2},
\end{aligned}
$$

where we have used the commutator estimate of Proposition 2.4. All the regularities of the involved objects are enough to take the limit in the product, as soon as $I\left(\left(X^{\varepsilon}\right)^{\diamond 2}\right) \circ\left(X^{\varepsilon}\right)^{\diamond 2}$ and $I\left(\left(X^{\varepsilon}\right)^{\diamond 3}\right) \circ\left(X^{\varepsilon}\right)^{\diamond 2}$ converges in the prescribed space. Unfortunately, as shown in Section 4, this is not true. However, the convergence holds after making a renormalization procedure and this is where the constant $C_{2}^{\varepsilon}$ takes its role. More precisely, we will consider the following stochastic terms:

$$
\left(I\left(\left(X^{\varepsilon}\right)^{\diamond 2}\right) \circ\left(X^{\varepsilon}\right)^{\diamond 2}\right)^{\diamond}=I\left(\left(X^{\varepsilon}\right)^{\diamond 2}\right) \circ\left(X^{\varepsilon}\right)^{\diamond 2}-C_{2}^{\varepsilon}
$$

and

$$
\left(I\left(\left(X^{\varepsilon}\right)^{\diamond 3}\right) \circ\left(X^{\varepsilon}\right)^{\diamond 2}\right)^{\diamond}=I\left(\left(X^{\varepsilon}\right)^{\diamond 3}\right) \circ\left(X^{\varepsilon}\right)^{\diamond 2}-3 C_{2}^{\varepsilon} X^{\varepsilon},
$$

with

$$
C_{2}^{\varepsilon}=\left.\mathbb{E}\left[I\left(\left(X^{\varepsilon}\right)^{\diamond 2}\right)(t) \circ\left(X^{\varepsilon}\right)^{\diamond 2}(t)\right]\right|_{t=0} .
$$

Making such a consideration pushes us to consider the term $\left(\Phi^{\varepsilon}\right) \circ\left(X^{\varepsilon}\right)^{\diamond 2}-$ $3 C_{2}^{\varepsilon}\left(X^{\varepsilon}+\Phi^{\varepsilon}\right)$ instead of the original one where we have added the extra counterpart introduced in equation (2.3), and at this point, we can see that this term has the following expansion:

$$
\begin{aligned}
\left(\Phi^{\varepsilon}\right) \circ & \left(X^{\varepsilon}\right)^{\diamond 2}-3 C_{2}^{\varepsilon}\left(X^{\varepsilon}+\Phi^{\varepsilon}\right) \\
= & I\left(\left(X^{\varepsilon}\right)^{\diamond 3}\right) \circ\left(X^{\varepsilon}\right)^{\diamond 2}-3 C_{2}^{\varepsilon} X^{\varepsilon} \\
& +3\left(\left(\Phi^{\varepsilon}\right)^{\prime}\left(I\left(\left(X^{\varepsilon}\right)^{\diamond 2}\right) \circ\left(X^{\varepsilon}\right)^{\diamond 2}\right)-C_{2}^{\varepsilon} \Phi^{\varepsilon}\right) \\
& +R\left(\left(X^{\varepsilon}\right)^{\diamond 2},\left(\Phi^{\varepsilon}\right)^{\prime}, I\left(\left(X^{\varepsilon}\right)^{\diamond 2}\right)\right)+\left(\Phi^{\varepsilon}\right)^{\natural} \circ\left(X^{\varepsilon}\right)^{\diamond 2} .
\end{aligned}
$$

It is important to remember that $\Phi^{\varepsilon}$ is expected to be a fixed point of the equation. In that setting, one must note that $\Phi^{\varepsilon}=\left(\Phi^{\varepsilon}\right)^{\prime}$. The following proposition is a summary of the discussion above (a rigorous proof of it, and more precise estimates can be found in Section 3.2).

Proposition 2.12. Suppose that $\Phi^{\varepsilon}$ fulfills Ansatz 2.11, then $\Phi^{\varepsilon}\left(X^{\varepsilon}\right)^{\diamond 2}-$ $3 C_{2}^{\varepsilon}\left(X^{\varepsilon}+\Phi^{\varepsilon}\right)$ is a continuous functional, uniformly in $\varepsilon$, of

$$
\begin{aligned}
& \Phi^{\varepsilon}, \quad\left(\Phi^{\varepsilon}\right)^{\prime}, \quad X^{\varepsilon}, \quad\left(X^{\varepsilon}\right)^{\diamond 2}, \quad I\left(\left(X^{\varepsilon}\right)^{\diamond 3}\right), \\
& \left(I\left(\left(X^{\varepsilon}\right)^{\diamond 2}\right) \circ\left(X^{\varepsilon}\right)^{\diamond 2}\right)^{\diamond} \quad \text { and } \quad\left(I\left(\left(X^{\varepsilon}\right)^{\diamond 3}\right) \circ\left(X^{\varepsilon}\right)^{\diamond 2}\right)^{\diamond} .
\end{aligned}
$$

The following corollary is a byproduct of those two propositions. 
COROllary 2.13. Let $\Phi^{\varepsilon}$ be as in Ansatz 2.11 and

$$
\begin{aligned}
\mathbb{X}^{\varepsilon}= & \left(X^{\varepsilon},\left(X^{\varepsilon}\right)^{\diamond 2}, I\left(\left(X^{\varepsilon}\right)^{\diamond 3}\right), I\left(\left(X^{\varepsilon}\right)^{\diamond 3}\right) \circ X^{\varepsilon},\right. \\
& \left.\left(I\left(\left(X^{\varepsilon}\right)^{\diamond 2}\right) \circ\left(X^{\varepsilon}\right)^{\diamond 2}\right)^{\diamond},\left(I\left(\left(X^{\varepsilon}\right)^{\diamond 3}\right) \circ\left(X^{\varepsilon}\right)^{\diamond 2}\right)^{\diamond}\right) .
\end{aligned}
$$

The function $\Gamma$ is continuous toward $\Phi^{\varepsilon},\left(\Phi^{\varepsilon}\right)^{\prime}$ and $\mathbb{X}^{\varepsilon}$, uniformly in $\varepsilon$, where

$$
\begin{aligned}
\Gamma\left(\Phi^{\varepsilon}\right)= & I\left(\left(X^{\varepsilon}\right)^{3}\right)+3 I\left(\left(X^{\varepsilon}\right)^{2} \Phi^{\varepsilon}\right)+I\left(X^{\varepsilon}\left(\Phi^{\varepsilon}\right)^{2}\right)+I\left(\left(\Phi^{\varepsilon}\right)^{3}\right) \\
& -3\left(C_{1}^{\varepsilon}+C_{2}^{\varepsilon}+C_{3}\right) I\left(X^{\varepsilon}+\Phi^{\varepsilon}\right) .
\end{aligned}
$$

As mentioned above, the aim of Sections 3.1 and 3.2 is to specify the dependency toward the parameters of the problem. In Section 3.3, these estimates will allow us to prove that in a suitable space (the space of paracontrolled distribution of Definition 3.3) and for a suitable $\mathbb{X}$ (lying in the space of rough distributions of Definition 2.15) $\Gamma$ is a contraction. This will allow us to make a fixed-point argument. Finally, in Section 4, we apply this analytical theory to the white noise and to $X$.

REMARK 2.14. Let us remark that the previous analysis for $\Phi^{\varepsilon}$ leads to the following corresponding problem for $u^{\varepsilon}$ :

$$
\partial_{t} u^{\varepsilon}=\Delta u^{\varepsilon}-\left(\left(u^{\varepsilon}\right)^{3}-3\left(C_{1}^{\varepsilon}+3 C_{2}^{\varepsilon}+C_{3}\right) u^{\varepsilon}\right)+\xi^{\varepsilon} .
$$

In all of the following, we will choose to take $C_{3}=0$ to simplify our presentation. The case $C_{3} \neq 0$ would only leads to a minor algebraic modification.

2.3. Rough distributions. To end this section, let us introduce the space $\mathcal{X}$ in which the convergence of $\mathbb{X}^{\varepsilon}$ takes place. The previous subsection gives us the structure of the data so that we are able to solve equation (1.1).

Definition 2.15. Let $T>1, v, \rho>0$. We denote by $\mathscr{C}_{T}^{v, \delta^{\prime}, \beta}$ the closure of the set of smooth functions $C^{\infty}\left([0, T], \mathcal{C}^{\beta}\left(\mathbb{T}^{3}\right)\right)$ by the semi-norm

$$
\|\varphi\|_{\nu, \rho}=\sup _{t \in[0, T]} t^{\nu}\left|\varphi_{t}\right|_{\mathcal{C}^{\beta}}+\sup _{t, s \in[0, T] ; s \neq t} \frac{s^{\nu}\left|\varphi_{t}-\varphi_{s}\right|_{\mathcal{C}^{\beta}}}{|t-s|^{\delta^{\prime}}}
$$

For $0<4 \delta^{\prime}<\delta$, we define the normed Banach space $\mathcal{W}_{T, K}$

$$
\mathcal{W}_{T, K}=C_{T}^{\delta^{\prime},-1 / 2-\delta} \times C_{T}^{\delta^{\prime},-1-\delta} \times C_{T}^{\delta^{\prime}, 1 / 2-\delta} \times C_{T}^{\delta^{\prime},-\delta} \times \mathscr{C}_{T}^{\nu, \delta^{\prime},-\delta} \times \mathscr{C}_{T}^{\nu, \delta^{\prime},-1 / 2-\delta},
$$

where $K=\left(\delta, \delta^{\prime}, v, \rho\right)$ is equipped with the product topology. For $X \in C([0, T]$, $\left.C\left(\mathbb{T}^{3}\right)\right)$, and $(a, b) \in \mathbb{R}^{2}$ we define $R_{a, b} X \in \mathcal{W}_{T, K}$ by

$$
\begin{aligned}
R_{a, b} X= & \left(X, X^{2}-a, I\left(X^{3}-3 a X\right), I\left(X^{3}-3 a X\right) \circ X,\right. \\
& \left.I\left(X^{2}-a\right) \circ\left(X^{2}-a\right)-b, I\left(X^{3}-3 a X\right) \circ\left(X^{2}-a\right)-3 b X\right) .
\end{aligned}
$$


The space of rough distributions $\mathcal{X}_{T, K}$ is defined as the closure of the set

$$
\left\{R_{a, b} X, X \in C\left([0, T], C\left(\mathbb{T}^{3}\right)\right) ;(a, b) \in \mathbb{R}^{2}\right\}
$$

in $\mathcal{W}_{T, K}$.

For a generic element $\mathbb{X} \in \mathcal{X}$, we denote its components by

$$
\mathbb{X}=\left(X, X^{\diamond 2}, I\left(X^{\diamond 3}\right), I\left(X^{\diamond 3}\right) \circ X,\left(I\left(X^{\diamond 2}\right) \circ X^{\diamond 2}\right)^{\diamond},\left(I\left(X^{\diamond 3}\right) \circ X^{\diamond 2}\right)^{\diamond}\right) .
$$

We equip the space $\mathcal{X}_{T, K}$ by the metric $\mathrm{d}$ induced by the topology of the Banach space $\mathcal{W}_{T, K}$ and we denote simply by $\|\mathbb{X}\|_{T, K}$ the norm of $\mathbb{X}$ in the space $\mathcal{W}_{T, K}$. For simplicity, we will omit in the sequel the dependencies in $T$ and $K$ for the space defined above and simply write $\mathcal{X}$.

REMARK 2.16. For $\mathbb{X} \in \mathcal{X}_{T, K}$, we can give a natural meaning to the the term $\left(I\left(X^{\diamond 2}\right) X^{\diamond 2}\right)^{\diamond}$ by using the Bony paraproduct decomposition:

$$
\left(I\left(X^{\diamond 2}\right) X^{\diamond 2}\right)^{\diamond}=I\left(X^{\diamond 2}\right) \prec X^{\diamond 2}+I\left(X^{\diamond 2}\right) \succ X^{\diamond 2}+\left(I\left(X^{\diamond 2}\right) \circ X^{\diamond 2}\right)^{\diamond} .
$$

In the same manner, we could also define $\left(I\left(X^{\diamond 3}\right) X^{\diamond 2}\right)^{\diamond}$.

REMARK 2.17. In the sequel, we might denote $\mathbb{X}$ by $X$ if there is no confusion.

Now let us summarize the discussion and give some pointers for the upcoming sections. First, in Section 3 we will introduce the space of paracontrolled distributions, which is formally speaking the space of distributions (actually a couple of distributions) such that the Ansatz 2.11 holds. In a second step, in Section 3.1 (resp., Section 3.2), we will show that given a fixed rough distribution $\mathbb{X}$ and a fixed paracontrolled distribution $\Phi$ we can construct the product $\Phi^{2} \diamond X^{\diamond}$ (resp., $\Phi \diamond X^{\diamond 2}$ ) like a continuous functional of the paracontrolled distribution $\Phi$ and the rough distribution $\mathbb{X}$. Moreover, this construction will coincide with the "classical"4 definitions when all data are smooth. Finally, in Section 3.3 we will show that for a small time the map $\Gamma$ is a contraction from the space of paracontrolled distributions to itself, which will allow us to construct immediately the map $\tilde{F}$ appearing in Theorem 1.1. It is wise to remark that all these parts are purely analytic and use simply the fact that $\mathbb{X}$ is a rough distribution. In order to come back to the original problem, we will prove in Section 4 that if $X^{\varepsilon}$ is a regularization of the (O.U), then $R_{C_{1}^{\varepsilon}, C_{2}^{\varepsilon}} X^{\varepsilon}=\mathbb{X}^{\varepsilon}$ converges in the space $\mathcal{X}_{T, K}$.

\footnotetext{
${ }^{4}$ Classical mean that the products appearing in this expression are understood in the usual sense of pointwise products of functions.
} 
3. Paracontrolled distributions and fixed-point equation. The aim of this section is to define a suitable space in which it is possible to formulate a fixed point for the eventual limit of the mollified solution. To be more precise, let $\mathbb{X}$ be a generic element of the space $\mathcal{X}$ (not necessarily equal to a fixed trajectory of the O.U.). We know from Section 4 that there exists $X^{\varepsilon} \in C_{T}^{1}\left(\mathbb{T}^{3}\right)$ and $a^{\varepsilon}, b^{\varepsilon} \in \mathbb{R}$ such that $\lim _{\varepsilon \rightarrow 0} R_{a^{\varepsilon}, b^{\varepsilon}} X^{\varepsilon}=\mathbb{X}$. Let us focus on the regular equation given by

$$
\begin{aligned}
\Phi^{\varepsilon}= & I\left(\left(X^{\varepsilon}\right)^{3}-3 a^{\varepsilon} X^{\varepsilon}\right) \\
& +3\left\{I\left(\Phi^{\varepsilon}\left(\left(X^{\varepsilon}\right)^{2}-a^{\varepsilon}\right)\right)-3 b^{\varepsilon} I\left(X^{\varepsilon}+\Phi^{\varepsilon}\right)\right\}+3 I\left(\left(\Phi^{\varepsilon}\right)^{2} X^{\varepsilon}\right)+I\left(\left(\Phi^{\varepsilon}\right)^{3}\right),
\end{aligned}
$$

where we have omitted temporarily the dependence on the initial condition. As pointed out previously, if we assume simply that $\Phi^{\varepsilon}$ converges to some $\Phi$ in $\mathcal{C}^{1 / 2-\delta}$, we see that the regularity of $\mathbb{X}$ is not sufficient to define $I\left(\Phi^{2} \diamond X\right):=$ $\lim _{\varepsilon \rightarrow 0} I\left(\left(\Phi^{\varepsilon}\right)^{2} X^{\varepsilon}\right)$ and $I\left(\Phi \diamond X^{\diamond 2}\right):=\lim _{\varepsilon \rightarrow 0} I\left(\Phi^{\varepsilon}\left(\left(X^{\varepsilon}\right)^{2}-a^{\varepsilon}\right)\right)+3 b^{\varepsilon} I\left(X^{\varepsilon}+\right.$ $\left.\Phi^{\varepsilon}\right)$. As it has been remarked in the previous section, the solution should satisfy the following decomposition:

$$
\Phi^{\varepsilon}=I\left(\left(X^{\varepsilon}\right)^{3}-3 a^{\varepsilon} X^{\varepsilon}\right)+3 I\left(\Phi^{\varepsilon} \prec\left(\left(X^{\varepsilon}\right)^{2}-a^{\varepsilon}\right)\right)+\left(\Phi^{\varepsilon}\right)^{\sharp} .
$$

Then if we impose the convergence of $\left(\Phi^{\varepsilon}\right)^{\sharp}$ to some $\Phi^{\sharp}$ in $C_{T}^{3 / 2-\delta}$, we see that the limit $\Phi$ should satisfy the following relation:

$$
\Phi^{\sharp}:=\Phi-I\left(X^{\diamond 3}\right)-3 I\left(\Phi \prec X^{\diamond 2}\right) \in C_{T}^{3 / 2-\delta},
$$

which as pointed in the previous section is the key point to define $I\left(\Phi^{2} X\right), I(\Phi \diamond$ $X^{\diamond 2}$ ) and to solve the equation

$$
\Phi=I\left(X^{\diamond 3}\right)+3 I\left(\Phi^{2} \diamond X\right)+3 I\left(\Phi \diamond X^{\diamond 2}\right)+I\left(\Phi^{3}\right) .
$$

Notation 3.1. Let us introduce some useful notations for the sequel:

$$
B_{>}(f, g)=I(f \succ g), \quad B_{0}(f, g)=I(f \circ g) \quad \text { and } \quad B_{<}(f, g)=I(f \prec g) \text {. }
$$

REMARK 3.2. The reader should keep in mind that the paraproduct $B_{<}(f, g)$ is always well defined for every $f \in \mathcal{C}^{\alpha}$ and $g \in \mathcal{C}^{\beta}$ for all the value of $\alpha$ and $\beta$ Moreover, it has regularity $\min (\alpha, \beta)+2-\delta$, for all $\delta>0$.

Now the following definition offers a precise meaning to the notion of paracontrolled distribution.

Definition 3.3. Let $\mathbb{X} \in \mathcal{X}$ and $z \in(1 / 2,2 / 3)$. We say that a couple $\left(\Phi, \Phi^{\prime}\right) \in\left(C_{T}^{-z}\right)^{2}$ is controlled by $\mathbb{X}$ if

$$
\Phi^{\sharp}=\Phi-I\left(X^{\diamond 3}\right)-3 B_{<}\left(\Phi^{\prime}, X^{\diamond 2}\right)
$$


such that

$$
\begin{aligned}
\left\|\Phi^{\sharp}\right\|_{\star, 1, L, T}= & \sup _{t \in[0, T]}\left(t^{\frac{1+\delta+z}{2}}\left\|\Phi_{t}^{\sharp}\right\|_{1+\delta}+t^{1 / 4+\frac{\gamma+z}{2}}\left\|\Phi_{t}^{\sharp}\right\|_{1 / 2+\gamma}+t^{\frac{\kappa+z}{2}}\left\|\Phi_{t}^{\sharp}\right\|_{\kappa}\right) \\
& +\sup _{(s, t) \in[0, T]^{2}} s^{\frac{z+a}{2}} \frac{\left\|\Phi_{t}^{\sharp}-\Phi_{s}^{\sharp}\right\|_{a-2 b}}{|t-s|^{b}}<+\infty
\end{aligned}
$$

and

$$
\left\|\Phi^{\prime}\right\|_{\star, 2, L, T}=\sup _{(s, t) \in[0, T]^{2}} s^{\frac{z+c}{2}} \frac{\left\|\Phi_{t}^{\prime}-\Phi_{s}^{\prime}\right\|_{c-2 d}}{|t-s|^{d}}+\sup _{t \in[0, T]} t^{\frac{\eta+z}{2}}\left\|\Phi_{t}^{\prime}\right\|_{\eta}<+\infty,
$$

where $L:=(\delta, \gamma, \kappa, a, b, c, d, \eta) \in[0,1]^{8}, z \in(1 / 2,2 / 3)$ and $2 d \leq c, 2 b \leq a$. Let us denote by $\mathcal{D}_{T, \mathbb{X}}^{L}$ the space of such couples of distributions. In order to keep it simple for the rest of the paper, we will use the abusive notation $\Phi$ instead of $\left(\Phi, \Phi^{\prime}\right)$. Moreover, we equip this space with the following metric:

$$
d_{L, T}\left(\Phi_{1}, \Phi_{2}\right)=\left\|\Phi_{1}^{\prime}-\Phi_{2}^{\prime}\right\|_{\star, 2, L, T}+\left\|\Phi_{1}^{\sharp}-\Phi_{2}^{\sharp}\right\|_{\star, 1, L, T}
$$

for $\Phi_{1}, \Phi_{2} \in \mathcal{D}_{T, \mathbb{X}}^{L}$ and the quantity

$$
\|\Phi\|_{\star, T, L}=\left\|\Phi_{1}\right\|_{\mathcal{D}_{T, X}^{L}}=d_{L, T}\left(\Phi_{1}, I\left(X^{\diamond 3}\right)\right) .
$$

REMARK 3.4. The metric space $\mathcal{D}_{T, \mathbb{X}}^{L}$ is complete.

In the following, we will omit $L$ when its choice is clear. We notice that the distance and the metric introduced in this last definition do not depend on $\mathbb{X}$. More generally, for $\Phi \in \mathcal{D}_{T_{1}, X}^{L}$ and $\Psi \in \mathcal{D}_{T_{2}, Y}^{G}$ we denote by $d_{\min (L, G), \min \left(T_{1}, T_{2}\right)}(\Phi, \Psi)$ the same quantity. We claim that if $\Phi \in \mathcal{D}_{X}^{L}$ for a suitable choice of $L$ then we are able to define $I\left(\Phi \diamond X^{\diamond 2}\right)$ and $I\left(\Phi^{2} \diamond X\right)$ modulo the use of $\mathbb{X}$.

In the following two subsections, we show respectively that $I\left(\Phi \diamond X^{\diamond 2}\right)$ and $I\left(\Phi^{2} X\right)$ are well defined when $\Phi$ is a controlled distribution. We also have to prove that when $\Phi$ is a controlled distribution, $\Psi+I\left(X^{\diamond 3}\right)+3 I\left(\Phi^{2} \diamond X\right)+3 I(\Phi \diamond$ $\left.X^{\diamond 2}\right)+I\left(\Phi^{3}\right)$ is also a controlled distribution. After all those verifications have been made, the only remaining point will be to show that we can apply a fixedpoint argument to find a solution to the renormalized equation. This is the aim of Section 3.3.

3.1. Decomposition of $I\left(\Phi^{2} \diamond X\right)$. Let $\mathbb{X} \in \mathcal{X}$ and $\Phi \in \mathcal{D}_{\mathbb{X}, T}^{L}$, assuming that all the components of $\mathbb{X}$ are smooth and using the fact that $\Phi$ is controlled we get immediately the following expansion:

$$
I\left(\Phi^{2} X\right)=I\left(I\left(X^{\diamond 3}\right)^{2} X\right)+I\left(\left(\theta^{\sharp}\right)^{2} X\right)+2 I\left(\theta^{\sharp} I\left(X^{\diamond 3}\right) X\right),
$$


where

$$
\theta^{\sharp}=B_{<}\left(\Phi^{\prime}, X^{\diamond 2}\right)+\Phi^{\sharp}
$$

When $\mathbb{X}$ is no longer smooth and only satisfies $\mathbb{X} \in \mathcal{X}$ and more particularly when $I\left(X^{\diamond 3}\right) \in C_{T}^{1 / 2-\delta}$ and $X \in C_{T}^{-1 / 2-\delta}$, we can observe that the two terms $I\left(\left(\theta^{\sharp}\right)^{2} X\right)$ and $I\left(\theta^{\sharp} I\left(X^{\diamond 3}\right) X\right)$ are well defined due to the Bony estimate (Proposition 2.3) and the fact that $\Phi$ is a paracontrolled distribution. Let us focus on the term $I\left(X^{\diamond 3}\right)^{2} X$ which, at this stage, is not well understood. However, the Bony paraproduct decomposition gives

$$
\begin{aligned}
I\left(X^{\diamond 3}\right)^{2} X= & \left(I\left(X^{\diamond 3}\right) \prec I\left(X^{\diamond 3}\right)\right) \circ X+\left(I\left(X^{\diamond 3}\right) \circ I\left(X^{\diamond 3}\right)\right) \circ X+I\left(X^{\diamond 3}\right)^{2} \prec X \\
& +I\left(X^{\diamond 3}\right)^{2} \succ X .
\end{aligned}
$$

We remark that only the first term of this expansion is not well defined. To overcome this problem, we use Proposition 2.4. Indeed we have

$$
R\left(I\left(X^{\diamond 3}\right), I\left(X^{\diamond 3}\right), X\right)=\left(I\left(X^{\diamond 3}\right) \prec I\left(X^{\diamond 3}\right)\right) \circ X-I\left(X^{\diamond 3}\right)\left(I\left(X^{\diamond 3}\right) \circ X\right),
$$

which is well defined and lies in the space $C_{T}^{1 / 2-3 \delta}$, since $\mathbb{X} \in \mathcal{X}$.

REMARK 3.5. The "extension" of the term $I\left(\Phi^{2} X\right)$ is a functional of $(\Phi, \mathbb{X}) \in \mathcal{D}_{\mathbb{X}, T}^{L} \times \mathcal{X}$ and then we use sometimes the notation $I\left(\Phi^{2} \diamond \mathbb{X}\right)[\Phi, \mathbb{X}]$ to underline this fact.

Proposition 3.6. Let $z \in(1 / 2,2 / 3), \Phi \in \mathcal{D}_{\mathbb{X}}^{L}$, and assume that $\mathbb{X} \in \mathcal{X}$. The quantity $I\left(\Phi^{2} \diamond X\right)[\Phi, \mathbb{X}]$ is well defined via the following expansion:

$$
I\left(\Phi^{2} \diamond X\right)[\Phi, \mathbb{X}]:=I\left(I\left(X^{\diamond 3}\right)^{2} X\right)+I\left(\left(\theta^{\sharp}\right)^{2} X\right)+2 I\left(\theta^{\sharp} I\left(X^{\diamond 3}\right) X\right),
$$

where

$$
\theta^{\sharp}=B_{<}\left(\Phi^{\prime}, X^{\diamond 2}\right)+\Phi^{\sharp}
$$

and

$$
\begin{aligned}
I\left(X^{\diamond 3}\right)^{2} X:= & I\left(X^{\diamond 3}\right) \circ I\left(X^{\diamond 3}\right) X+2\left(I\left(X^{\diamond 3}\right) \prec I\left(X^{\diamond 3}\right)\right) \prec X \\
& +2\left(I\left(X^{\diamond 3}\right) \prec I\left(X^{\diamond 3}\right)\right) \succ X \\
& +2 I\left(X^{\diamond 3}\right) I\left(X^{\diamond 3}\right) \circ X+2 R\left(I\left(X^{\diamond 3}\right), I\left(X^{\diamond 3}\right), X\right) .
\end{aligned}
$$

Here,

$$
R\left(I\left(X^{\diamond 3}\right), I\left(X^{\diamond 3}\right), X\right)=\left(I\left(X^{\diamond 3}\right) \prec I\left(X^{\diamond 3}\right)\right) \circ X-I\left(X^{\diamond 3}\right)\left(I\left(X^{\diamond 3}\right) \circ X\right)
$$

is well defined by Proposition 2.4. Moreover, there exists a choice of $L$ such that the following bound holds:

$$
\left\|I\left(\Phi^{2} \diamond X\right)[\Phi, \mathbb{X}]\right\|_{\star, 1, T} \lesssim T^{\theta}\left(\|\Phi\|_{\mathcal{D}_{X}^{L}}+1\right)^{2}\left(1+\|\mathbb{X}\|_{T, \nu, \rho, \delta, \delta^{\prime}}\right)^{3},
$$


for $\theta>0$ and $\delta, \delta^{\prime}, \rho, v>0$ small enough depending on $L$ and $z$. Moreover, if $X \in C_{T}^{1}\left(\mathbb{T}^{3}\right)$ then

$$
I\left(\Phi^{2} \diamond X\right)\left[\Phi, R_{a, b} X\right]=I\left(\Phi^{2} X\right)
$$

PROOF. By a simple computation, we have

$$
\begin{aligned}
\left\|B_{<}\left(\Phi^{\prime}, X^{\diamond 2}\right)(t)\right\|_{\kappa} & \lesssim \int_{0}^{t} \mathrm{~d} s(t-s)^{-(\kappa+1+r) / 2}\left\|\Phi_{s}^{\prime}\right\|_{\kappa}\left\|X_{s}^{\diamond 2}\right\|_{-1-r} \\
& \lesssim_{r, \kappa} T^{1 / 2-r / 2-\kappa / 2-z / 2}\left\|\Phi^{\prime}\right\|_{\star, 2, T}\left\|X^{\diamond 2}\right\|_{-1-r},
\end{aligned}
$$

for $r, \kappa>0$ small enough and $1 / 2<z<2 / 3$. A similar computation gives

$$
\begin{aligned}
\| B_{<} & \left(\Phi^{\prime}, X^{\diamond 2}\right)(t) \|_{1 / 2+\gamma} \\
& \lesssim \int_{0}^{t} \mathrm{~d} s(t-s)^{-(3 / 2+\gamma+r) / 2}\left\|\Phi_{s}^{\prime}\right\|_{\kappa}\left\|X_{s}^{\diamond 2}\right\|_{-1-r} \\
& \lesssim \kappa, r, z\left\|\Phi^{\prime}\right\|_{\star, 2, T}\left\|X^{\diamond 2}\right\|_{-1-r} \int_{0}^{t} \mathrm{~d} s(t-s)^{-(3 / 2+\gamma+r) / 2} s^{-(\kappa+z) / 2} \\
& \lesssim t^{1 / 4-(\gamma+\kappa+z+r) / 2}\left\|\Phi^{\prime}\right\|_{\star, 2, L, T}\left\|X^{\diamond 2}\right\|_{-1-r},
\end{aligned}
$$

for $\gamma, r, \kappa>0$ small enough. Using this bound, we can deduce that

$$
\begin{aligned}
\left\|I\left(\left(\theta^{\sharp}\right)^{2} X\right)(t)\right\|_{1+\delta} & \vdots \int_{0}^{t} \mathrm{~d} s(t-s)^{-(3 / 2+\delta+\beta) / 2}\left\|\left(\theta_{s}^{\sharp}\right)^{2} X_{s}\right\|_{-1 / 2-\beta} \\
& \lesssim \beta, \delta \int_{0}^{t} \mathrm{~d} s(t-s)^{-(3 / 2+\delta+\beta) / 2}\left\|\theta_{s}^{\sharp}\right\|_{\kappa}\left\|\theta_{s}^{\sharp}\right\|_{1 / 2+\gamma}\left\|X_{S}\right\|_{-1 / 2-\beta} \\
& \vdots L, z\|\Phi\|_{\star, L, T}^{2}\left(\left\|X^{\diamond 2}\right\|_{-1-r}+\|X\|_{-1 / 2-\beta}+1\right)^{2} \\
& \times \int_{0}^{t} \mathrm{~d} s(t-s)^{-(3 / 2+\delta+\beta)} s^{-(1 / 2+\kappa+\gamma+2 z) / 2} \\
& \vdots L, z
\end{aligned}
$$

for $\gamma, \beta, \delta>0$ small enough and $2 / 3>z>1 / 2$. Hence we have

$$
\begin{aligned}
& \sup _{t \in[0, T]} t^{(1+\delta+z) / 2}\left\|I\left(\left(\theta^{\sharp}\right)^{2} X\right)(t)\right\|_{1+\delta} \\
& \quad \lesssim L T^{\theta_{1}}\|\Phi\|_{\star, L, T}^{2}\left(\left\|X^{\diamond 2}\right\|_{-1-r}+\|X\|_{-1 / 2-\beta}+1\right)^{2},
\end{aligned}
$$

for some $\theta_{1}>0$ depending on $L$ and $z$. The same type of computation gives

$$
\begin{aligned}
& \sup _{t \in[0, T]} t^{(\kappa+z) / 2}\left\|I\left(\left(\theta^{\sharp}\right)^{2} X\right)(t)\right\|_{\kappa} \\
& \quad \sum_{L, z} T^{\theta_{2}}\|\Phi\|_{\star, L, T}^{2}\left(\left\|X^{\diamond 2}\right\|_{-1-r}+\|X\|_{-1 / 2-\beta}+1\right)^{2}
\end{aligned}
$$


and

$$
\begin{aligned}
& \sup _{t \in[0, T]} t^{(1 / 2+\gamma+z) / 2}\left\|I\left(\left(\theta^{\sharp}\right)^{2} X\right)(t)\right\|_{1 / 2+\gamma} \\
& \quad \lesssim_{L, z} T^{\theta_{3}}\|\Phi\|_{\star, T}^{2}\left(\left\|X^{\diamond 2}\right\|_{-1-r}+\|X\|_{-1 / 2-\beta}+1\right)^{2},
\end{aligned}
$$

where $\theta_{2}$ and $\theta_{3}$ are two nonnegative constants depending only on $L$ and $z$. To complete our study for this term, we have also

$$
\left\|\left(\left(\theta^{\sharp}\right)^{2} X\right)(t)-I\left(\left(\theta^{\sharp}\right)^{2} X\right)(s)\right\|_{a-2 b} \lesssim I_{s t}^{1}+I_{s t}^{2},
$$

where

$$
\begin{aligned}
I_{s t}^{1} & =\left\|\int_{0}^{s} \mathrm{~d} u\left(P_{t-u}-P_{s-u}\right)\left(\theta_{u}^{\sharp}\right)^{2} X_{u}\right\|_{a-2 b} \text { and } \\
I_{s t}^{2} & =\left\|\int_{s}^{t} \mathrm{~d} u P_{t-u}\left(\theta_{u}^{\sharp}\right)^{2} X_{u}\right\|_{a-2 b} .
\end{aligned}
$$

Let us begin by bounding $I^{1}$ :

$$
\begin{aligned}
I_{s t}^{1} & \lesssim(t-s)^{b} \int_{0}^{s} \mathrm{~d} u\left\|P_{s-u}\left(\theta_{u}^{\sharp}\right)^{2} X_{u}\right\|_{a} \\
& \lesssim(t-s)^{b} \int_{0}^{t} \mathrm{~d} u(s-u)^{-(1 / 2+a+\beta)}\left\|\left(\theta_{u}^{\sharp}\right)^{2} X_{u}\right\|_{-1 / 2-\beta} \\
& \lesssim T^{\theta_{4}}|t-s|^{b}\|\Phi\|_{\star, T}^{2}\left(\left\|X^{\diamond 2}\right\|_{-1-r}+\|X\|_{-1 / 2-\beta}+1\right)^{2},
\end{aligned}
$$

where $\theta_{4}>0$ depending on $L$ and $z$. Let us focus on the bound for $I^{2}$,

$$
\begin{aligned}
I_{s t}^{2} \lesssim & \int_{s}^{t}(t-u)^{-(1 / 2+a-2 b+\beta) / 2}\left\|\left(\theta_{u}^{\sharp}\right)^{2} X_{u}\right\|_{-1 / 2-\beta} \\
\lesssim & L, z\|\Phi\|_{\star, T}^{2}\left(\left\|X^{\diamond 2}\right\|_{-1-r}+\|X\|_{-1 / 2-\beta}+1\right)^{2} \\
& \quad \times \int_{s}^{t} \mathrm{~d} u(t-u)^{-(1 / 2+a-2 b+\beta) / 2} u^{-(1 / 2+\kappa+\gamma+2 z) / 2}
\end{aligned}
$$

and

$$
\begin{aligned}
\int_{s}^{t} \mathrm{~d} u(t & -u)^{-(1 / 2+a-2 b+\beta) / 2} u^{-(1 / 2+\kappa+\gamma+2 z) / 2} \\
= & (t-s)^{3 / 4-(a-2 b+\beta)} \\
& \times \int_{0}^{1} \mathrm{~d} x(1-x)^{-(1 / 2+a-2 b+\beta)}(s+x(t-s))^{-(1 / 2+\kappa+\gamma+2 z) / 2} \\
\lesssim & l, \kappa, \gamma, a, b(t-s)^{l-(a-2 b+\beta) / 2} s^{1 / 2-z+(\kappa+\gamma) / 2} \\
& \times \int_{0}^{1} \mathrm{~d} x(1-x)^{-(1 / 2+a-2 b+\beta) / 2} x^{-3 / 4+l} .
\end{aligned}
$$


Since $z<1$, we can choose $l, \kappa, \gamma, b>0$ small enough and we have

$$
\int_{s}^{t} \mathrm{~d} u(t-u)^{-(1 / 2+a-2 b+\beta) / 2} u^{-(1 / 2+\kappa+\gamma+2 z) / 2} \lesssim L T^{\theta_{5}}(t-s)^{b} s^{-(z+a) / 2},
$$

where $\theta_{5}>0$. This gives the needed bound for $I_{2}$. Finally, we have

$$
\begin{gathered}
\sup _{(s, t) \in[0, T]} s^{(z+a) / 2} \frac{\left\|I\left(\left(\theta^{\sharp}\right)^{2} X\right)(t)-I\left(\left(\theta^{\sharp}\right)^{2} X\right)(s)\right\|_{a-2 b}}{|t-s|^{b}} \\
\quad \lesssim T^{\theta_{5}}\|\Phi\|_{\star, T}^{2}\left(\left\|X^{\diamond 2}\right\|_{-1-r}+\|X\|_{-1 / 2-\beta}+1\right)^{2} .
\end{gathered}
$$

Hence

$$
\left\|I\left(\left(\theta^{\sharp}\right)^{2} X\right)\right\|_{\star, 1, T} \lesssim_{L} T^{\theta}\|\Phi\|_{\star, T}^{2}\left(\left\|X^{\diamond 2}\right\|_{-1-r}+\|X\|_{-1 / 2-\beta}+1\right)^{2} .
$$

The bound for $\left\|I\left(\theta^{\sharp} I\left(X^{3}\right) X\right)\right\|_{\star, 1, T}$ can be obtained by a similar way and then, according to the hypothesis given on the area term $I\left(X^{\diamond 3}\right) X$ and the decomposition of $I\left(I\left(X^{\diamond 3}\right)^{2} X\right)$, we obtain from Proposition 2.4 and Proposition 2.3 that

$$
\begin{aligned}
& \left\|I\left(I\left(X^{\diamond 3}\right)\right)^{2} \diamond X\right\|_{\star, 1, T} \\
& \quad \lesssim T^{\theta}\left(1+\left\|I\left(X^{\diamond 3}\right) \circ X\right\|_{\delta^{\prime},-1 / 2-\rho}+\left\|I\left(X^{\diamond 3}\right)\right\|_{\delta^{\prime}, 1 / 2-\rho}+\|X\|_{\delta^{\prime},-1 / 2-\rho}\right)^{3},
\end{aligned}
$$

for $3 \rho<\delta^{\prime}$ small enough, which gives the wanted result.

3.2. Decomposition of $I\left(\Phi \diamond X^{\diamond 2}\right)$. First, let $X \in C\left([0, T], C^{\infty}\right),(a, b) \in \mathbb{R}^{2}$, $\mathbb{X}=R_{a, b} X$ and $\Phi \in \mathcal{D}_{R_{a, b} X, T}$. Using the paracontrolled structure of $\Phi$ and the Bony paraproduct decomposition for the term $\Phi\left(X^{2}-a\right)$ gives the following expansion:

$$
\begin{aligned}
& I\left(\Phi\left(X^{2}-a\right)\right)-3 b I\left(\Phi^{\prime}+X\right) \\
&=B_{<}\left(\Phi, X^{2}-a\right)+\left(B_{0}\left(I\left(X^{3}\right)-3 a X, X^{2}-a\right)-3 b I(X)\right) \\
&+3\left(B_{0}\left(B_{<}\left(\Phi^{\prime}, X^{2}-a\right), X^{2}-a\right)-3 b I\left(\Phi^{\prime}\right)\right) \\
&+B_{0}\left(\Phi^{\sharp}, X^{2}-a\right)+B_{>}\left(\Phi, X^{2}-a\right) .
\end{aligned}
$$

Thanks to the Bony estimate (Proposition 2.3), we can see that all the terms appearing in the right- hand side, apart from the third one, are well defined even when $\mathbb{X}$ is no more equal to $R_{a, b} X$ but a general rough distribution. The only problem is to give an expansion for the third term of this equation. We have to deal with the (ill-defined) diagonal term:

$$
\begin{aligned}
J\left(\Phi^{\prime},\right. & \left.R_{a, b} X\right)(t) \\
& =B_{0}\left(B_{<}\left(\Phi^{\prime}, X^{2}-a\right), X^{2}-a\right)(t)-b I\left(\Phi^{\prime}\right) \\
& =\int_{0}^{t} \mathrm{~d} s P_{t-s} \int_{0}^{s} \mathrm{~d} \sigma P_{s-\sigma}\left(\Phi_{\sigma}^{\prime} \prec\left(X_{\sigma}^{2}-a\right)\right) \circ\left(X_{s}^{2}-a\right)-3 b I\left(\Phi^{\prime}\right) .
\end{aligned}
$$


It can be decomposed in the following way:

$$
\begin{aligned}
J\left(\Phi^{\prime}, R_{a, b} X\right)(t)= & \int_{0}^{t} \mathrm{~d} s P_{t-s} \Phi_{s}^{\prime}\left(I\left(X^{2}-a\right)(s) \circ\left(X_{s}^{2}-a\right)-b\right) \\
& +\int_{0}^{t} \mathrm{~d} s P_{t-s} \int_{0}^{s} \mathrm{~d} \sigma\left(\Phi_{\sigma}^{\prime}-\Phi_{s}^{\prime}\right)\left(X_{s}^{2}-a\right) \circ P_{s-\sigma}\left(X_{\sigma}^{2}-a\right) \\
& +\int_{0}^{t} \mathrm{~d} s P_{t-s} \int_{0}^{s} \mathrm{~d} \sigma R_{s-\sigma}^{1}\left(\Phi_{\sigma}^{\prime}, X_{\sigma}^{2}-a\right) \circ\left(X_{s}^{2}-a\right) \\
& +\int_{0}^{t} \mathrm{~d} s P_{t-s} \int_{0}^{s} R^{2}\left(\Phi_{\sigma}^{\prime}, P_{s-\sigma} X_{\sigma}^{2}-a, X_{s}^{2}-a\right) \\
\equiv & \sum_{i=1}^{4} J_{i}\left(\Phi^{\prime}, R_{a, b} X\right)(t),
\end{aligned}
$$

where

$$
\begin{aligned}
& R_{s-\sigma}^{1}(f, g)=P_{s-\sigma}(f \prec g)-f \prec P_{s-\sigma} g, \\
& R^{2}(f, g, h)=(f \prec g) \circ h-f(g \circ h)
\end{aligned}
$$

and $f, g, h$ are distributions lying in the suitable Besov spaces where the order for $R^{1}$ and $R^{2}$ has to be defined. Now the point is that the right-hand side of this equation allows us to define the operator $J$ (and even each $J_{i}$ ) for a general rough distribution $\mathbb{X}$, as it will be proved in Proposition 3.8 below, and for a general $\mathbb{X}$. Before stating the proposition, let us give a useful improvement of the Schauder estimate which will help us to estimate the operator $J$.

LEMMA 3.7. Let $f$ be a space time distribution such that

$$
\sup _{t \in[0, T]} t^{(r+z) / 2}\left\|f_{t}\right\|_{r}<+\infty .
$$

Then the following bound holds:

$$
\sup _{s, t \in[0, T]} \frac{\|I(f)(t)-I(f)(s)\|_{a-2 b}}{|t-s|^{b}} \lesssim_{b, a, z, r} T^{\theta} \sup _{t \in[0, T]} t^{(r+z) / 2}\left\|f_{t}\right\|_{r},
$$

where $a+z<2, z+r<2, a-r<2,0<a, b<1$ and $\theta>0$ is a constant depending only on $a, r, b, z$.

Proof. By a simple computation, we have

$$
I(f)(t)-I(f)(s)=I_{s t}^{1}+I_{s t}^{2},
$$

where

$$
I_{s t}^{1}=\left(P_{t-s}-1\right) \int_{0}^{s} \mathrm{~d} u P_{s-u} f_{u} \quad \text { and } \quad I_{s t}^{2}=\int_{s}^{t} \mathrm{~d} u P_{t-u} f_{u}
$$


Using Lemma 2.5, the following bound holds:

$$
\left\|I_{s t}^{1}\right\|_{a-2 b} \lesssim|t-s|^{b} \int_{0}^{t} \mathrm{~d} u(t-u)^{-(a-r) / 2} u^{-(r+z) / 2} \sup _{t \in[0, T]} t^{(r+z) / 2}\left\|f_{t}\right\|_{r}<+\infty .
$$

To handle the second term, we use the Hölder inequality,

$$
\begin{aligned}
\left\|I_{s t}^{2}\right\|_{a-2 b} \lesssim & |t-s|^{b}\left(\int_{s}^{t} \mathrm{~d} u(t-u)^{\frac{-(a-2 b-r)}{2(1-b)}} u^{-\frac{(z+r)}{2(1-b)}}\right)^{1-b} \\
& \times \sup _{t \in[0, T]} t^{(r+z) / 2}\left\|f_{t}\right\|_{r}<+\infty
\end{aligned}
$$

which completes the proof.

The following proposition gives us the regularity for our terms.

Proposition 3.8. Assume that $X$ is smooth and that $\mathbb{X}=R_{a, b} X$. There is a choice of $L$ such that for all $z \in(1 / 2,2 / 3)$ the following bound holds:

$$
\left\|J\left(\Phi^{\prime}, \mathbb{X}\right)(t)\right\|_{\star, 1, T} \lesssim T^{\theta}\left(1+\|\mathbb{X}\|_{T, K}\right)^{2}\left\|\Phi^{\prime}\right\|_{\star, 2, T},
$$

where $K \in[0,1]^{4}$ and $\theta>0$ are two small parameters depending only on $L$ and $z$. Thus this bound allows us to extend the operator $J$ the whole space of rough distributions $\mathcal{X}$, with the same bound.

PROOF. We begin by estimating the first term of the expansion (3.3):

$$
\begin{aligned}
& \left\|J_{1}\left(\Phi^{\prime}, \mathbb{X}\right)(t)\right\|_{1+\delta} \\
& \quad \lesssim \int_{0}^{t} \mathrm{~d} s(t-s)^{-(1+\delta+\eta / 2) / 2}\left\|\Phi_{s}^{\prime}\left(I\left(X^{2}-a\right)(s)\right) \circ\left(X_{s}^{\diamond}-a\right)-b\right\|_{-\eta / 2} \\
& \lesssim \\
& \quad\left\|\Phi^{\prime}\right\|_{\star, 2, T}\left\|B_{0}\left(\left(X^{2}-a\right),\left(X^{2}-a\right)\right)-b\right\|_{\mathscr{C}_{T}^{-\eta / 2, v}} \\
& \quad \times\left(\int_{0}^{t} \mathrm{~d} s(t-s)^{-(1+\delta+\eta / 2) / 2} s^{-(\eta+v+z) / 2}\right) \\
& \quad \lesssim \beta, L \\
& T^{\theta_{1}}\left\|\Phi^{\prime}\right\|_{\star, 2, T}\left\|B_{0}\left(\left(X^{2}-a\right),\left(X^{2}-a\right)\right)-b\right\|_{\mathscr{C}_{T}^{-\eta / 2, v}},
\end{aligned}
$$

for $\eta, \delta>0$ small enough and where $\theta_{1}>0$ depends on $L$. Hence

$$
\begin{aligned}
& \sup _{t \in[0, T]} t^{(1+\delta+z) / 2}\left\|J_{1}\left(\Phi^{\prime}, \mathbb{X}\right)(t)\right\|_{1+\delta} \\
& \quad{ }_{L, z} T^{\theta_{1}}\left\|\Phi^{\prime}\right\|_{\star, 2, T}\left\|B_{0}\left(\left(X^{2}-a\right),\left(X^{2}-a\right)\right)\right\|_{\mathscr{C}_{T}^{-\eta / 2, v}}
\end{aligned}
$$


Let us focus on the second term. We have

$$
\begin{aligned}
& \left\|J_{2}\left(\Phi^{\prime}, \mathbb{X}\right)(t)\right\|_{1+\delta} \\
& \lesssim \int_{0}^{t} \mathrm{~d} s(t-s)^{-(1+\delta-\beta) / 2} \\
& \times \int_{0}^{s} \mathrm{~d} \sigma\left\|\left(\Phi_{\sigma}^{\prime}-\Phi_{s}^{\prime}\right)\left(P_{s-\sigma}\left(X_{\sigma}^{\diamond 2}-a\right) \circ\left(X_{s}^{2}-a\right)\right)\right\|_{\beta} \\
& \lesssim \beta, \rho \int_{0}^{t} \mathrm{~d} s(t-s)^{-(1+\delta-\beta) / 2} \\
& \times \int_{0}^{s} \mathrm{~d} \sigma(s-\sigma)^{-(2+\rho) / 2}\left\|\Phi_{\sigma}^{\prime}-\Phi_{s}^{\prime}\right\|_{c-2 d}\left\|X_{s}^{2}-a\right\|_{-1-\rho}^{2} \\
& \lesssim_{L, \beta, \rho}\left\|\Phi^{\prime}\right\|_{\star, 2, T}\left\|X^{2}-a\right\|_{C_{T}^{-1-\rho}}^{2} \int_{0}^{t} \mathrm{~d} s(t-s)^{-(1+\delta-\beta) / 2} \\
& \times \int_{0}^{s} \mathrm{~d} \sigma(s-\sigma)^{-1-\rho / 2+d} \sigma^{-(c+z) / 2} \\
& \lesssim_{L, \beta, \rho}\left\|\Phi^{\prime}\right\|_{\star, 2, T}\left\|X^{2}-a\right\|_{C_{T}^{-1-\rho}}^{2} \int_{0}^{t} \mathrm{~d} s(t-s)^{-(1+\delta-\beta) / 2} s^{-(\rho+c-2 d+z) / 2} \\
& \lesssim_{L, \beta, \rho} T^{\theta_{2}}\left\|\Phi^{\prime}\right\|_{\star, 2, T}\left\|X^{2}-a\right\|_{C_{T}^{-1-\rho}}^{2},
\end{aligned}
$$

where $\beta=\min (c-2 d, \rho) \geq 0, c, d, \rho>0$ are small enough; $z<1$ and $\theta_{2}>0$ are a constant which depends only on $L$ and $z$. Using Lemma 2.5 have

$$
\left\|R_{s-\sigma}^{1}\left(\Phi_{\sigma}^{\prime}, X_{\sigma}^{2}-a\right)\right\|_{1+2 \beta} \lesssim(s-\sigma)^{-(2+3 \beta-\eta) / 2}\left\|\Phi_{\sigma}^{\prime}\right\|_{\eta}\left\|X_{\sigma}^{2}-a\right\|_{-1-\beta},
$$

for all $\beta>0, \beta<\eta / 3$ small enough. By a straightforward computation, we have

$$
\begin{aligned}
\left\|J_{3}\left(\Phi^{\prime}, \mathbb{X}\right)(t)\right\|_{1+\delta} & \vdots \int_{0}^{t} \mathrm{~d} s(t-s)^{-(1+\delta-\beta) / 2} \int_{0}^{s} \mathrm{~d} \sigma\left\|R_{s-\sigma}^{1}\left(\Phi_{\sigma}^{\prime}, X_{\sigma}^{2}-a\right) \circ\left(X_{s}^{2}-a\right)\right\|_{\beta} \\
\lesssim & \int_{0}^{t} \mathrm{~d} s(t-s)^{-(1+\delta-\beta) / 2} \int_{0}^{s} \mathrm{~d} \sigma\left\|R_{s-\sigma}^{1}\left(\Phi_{\sigma}^{\prime}, X_{\sigma}^{2}\right)-a\right\|_{1+2 \beta}\left\|X_{s}^{2}-a\right\|_{-1-\beta} \\
\lesssim & \left\|X^{2}-a\right\|_{C_{T}^{-1-\beta}}^{2}\left\|\Phi^{\prime}\right\|_{\star, 2, T} \int_{0}^{t} \mathrm{~d} s(t-s)^{-(1+\delta-\beta) / 2} \\
& \times \int_{0}^{s} \mathrm{~d} \sigma(s-\sigma)^{-(2+3 \beta-\kappa) / 2} \sigma \\
& \lesssim\left\|X^{2}-a\right\|_{C_{T}^{-1-\beta}}^{2}\left\|\Phi^{\prime}\right\|_{\star, 2, T} \int_{0}^{t} \mathrm{~d} s(t-s)^{-(1+\delta-\beta) / 2} s^{-(3 \beta-\kappa+\eta+z) / 2} \\
\lesssim & T^{\theta_{3}}\left\|X^{2}-a\right\|_{C_{T}^{-1-\beta}\left\|\Phi^{\prime}\right\|_{\star, 2, T}}^{2},
\end{aligned}
$$


where $\theta_{3}>0$ is a constant depending on $L$ and $z, 0<\beta<\eta / 3$ small enough and $z<1$. To treat the last term, it is sufficient to use the commutation result given in Proposition 2.4. Indeed we have

$$
\begin{aligned}
& \left\|R^{2}\left(\Phi_{\sigma}^{\prime}, P_{s-\sigma}\left(X_{\sigma}^{2}-a\right), X_{s}^{2}-a\right)\right\|_{\eta-3 \beta} \\
& \quad \lesssim \eta \eta, \beta s^{-(\eta+z) / 2}(s-\sigma)^{-(2-\beta) / 2}\left\|X^{2}-a\right\|_{C_{T}^{-1-\beta}}^{2}\left\|\Phi^{\prime}\right\|_{\star, 2, T},
\end{aligned}
$$

for $0<\beta<\eta / 3$ small enough. Hence

$$
\begin{aligned}
& \left\|J_{4}\left(\Phi^{\prime}, \mathbb{X}\right)(t)\right\|_{1+\delta} \\
& \lesssim_{\eta, \beta}\left\|X^{2}-a\right\|_{C_{T}^{-1-\beta}}^{2}\left\|\Phi^{\prime}\right\|_{\star, 2, T} \\
& \times \int_{0}^{t} \mathrm{~d} s(t-s)^{-(1+\delta-\eta+3 \beta) / 2} \int_{0}^{s} \mathrm{~d} \sigma s^{-(\eta+z) / 2}(s-\sigma)^{-(2-\beta) / 2} \\
& \lesssim_{\eta, \beta}\left\|X^{2}-a\right\|_{C_{T}^{-1-\beta}}^{2}\left\|\Phi^{\prime}\right\|_{\star, 2, T} \int_{0}^{t} \mathrm{~d} s(t-s)^{-(1+\delta-\eta+3 \beta) / 2} s^{-(\eta+z+\beta) / 2} \\
& \lesssim T^{\theta_{4}}\left\|X^{2}-a\right\|_{C_{T}^{-1-\beta}}^{2}\left\|\Phi^{\prime}\right\|_{\star, 2, T},
\end{aligned}
$$

for $\theta_{4}>0$ depending on $L$ and $z<1$ and $\beta, \eta, \delta>0$ small enough. Binding all these bounds together, we can conclude that

$$
\begin{aligned}
& \sup _{t \in[0, T]} t^{(1+\delta+z) / 2}\left\|J\left(\Phi^{\prime}, \mathbb{X}\right)(t)\right\|_{1+\delta} \\
& \quad \lesssim{ }_{L, z} T^{\theta}\left(1+\left\|X^{2}-a\right\|_{C_{T}^{-1-\rho}}+\left\|B_{0}\left(\left(X^{2}-a\right),\left(X^{2}-a\right)\right)-b\right\|_{\mathscr{C}_{T}^{-\eta / 2, \nu}}\right)^{2} \\
& \quad \times\left\|\Phi^{\prime}\right\|_{\star, 2, T},
\end{aligned}
$$

for $\theta>0$ depending on $L$ and $z$. The same arguments gives

$$
\begin{aligned}
& \sup _{t \in[0, T]} t^{(1 / 2+\gamma+z) / 2}\left\|J\left(\Phi^{\prime}, \mathbb{X}\right)(t)\right\|_{1 / 2+\gamma} \\
& \quad{ }_{L, z} T^{\theta}\left(1+\left\|X^{2}-a\right\|_{C_{T}^{-1-\rho}}+\left\|B_{0}\left(\left(X^{2}-a\right),\left(X^{2}-a\right)\right)-b\right\|_{\mathscr{C}_{T}^{-\eta / 2, \nu}}\right)^{2} \\
& \quad \times\left\|\Phi^{\prime}\right\|_{\star, 2, T}
\end{aligned}
$$

and

$$
\begin{aligned}
& \sup _{t \in[0, T]} t^{(\kappa+z) / 2}\left\|J\left(\Phi^{\prime}, \mathbb{X}\right)(t)\right\|_{\kappa} \\
& \quad \lesssim L, z T^{\theta}\left(1+\left\|X^{\diamond 2}\right\|_{C_{T}^{-1-\rho}}+\left\|B_{0}\left(\left(X^{2}-a\right),\left(X^{2}-a\right)\right)-b\right\|_{\mathscr{C}_{T}^{-\eta / 2, v}}\right)^{2} \\
& \quad \times\left\|\Phi^{\prime}\right\|_{\star, 2, T} .
\end{aligned}
$$


To obtain the needed bound, we still need to estimate the following quantity:

$$
\sup _{(s, t) \in[0, T]^{2}} s^{\frac{z+a}{2}} \frac{\left\|J\left(\Phi^{\prime}, \mathbb{X}\right)(t)-J\left(\Phi^{\prime}, \mathbb{X}\right)(s)\right\|_{a-2 b}}{|t-s|^{b}} .
$$

To handle this, we use the fact that $J_{i}\left(\Phi^{\prime}, \mathbb{X}\right)(t)=I\left(f^{i}\right)$ with

$$
\begin{aligned}
& f^{1}(s)=\Phi_{s}^{\prime} I\left(\left(X^{2}-a\right)(s) \circ\left(X_{s}^{\diamond}-a\right)-b\right), \\
& f^{2}(s)=\int_{0}^{s} \mathrm{~d} \sigma\left(\Phi_{\sigma}^{\prime}-\Phi_{s}^{\prime}\right)\left(\left(X_{s}^{2}-a\right) \circ P_{s-\sigma} X_{\sigma}^{2}-a\right)
\end{aligned}
$$

and

$$
\begin{aligned}
& f^{3}(s)=\int_{0}^{s} \mathrm{~d} \sigma\left(R_{s-\sigma}^{1}\left(\Phi_{\sigma}^{\prime}, X_{\sigma}^{2}-a\right) \circ\left(X_{s}^{2}-a\right)\right), \\
& f^{4}(s)=\int_{0}^{s} R^{2}\left(\Phi_{\sigma}^{\prime}, P_{s-\sigma}\left(X_{\sigma}^{2}-a\right), X_{s}^{2}-a\right) .
\end{aligned}
$$

By an easy computation, we have

$$
\begin{aligned}
\left\|f^{1}(t)\right\|_{\eta / 2} \lesssim \eta & s^{-(\eta+z) / 2}\left\|\Phi^{\prime}\right\|_{\star, 2, T} \\
& \times\left(1+\left\|B_{0}\left(\left(X^{2}-a\right),\left(X^{2}-a\right)\right)-b\right\|_{\mathscr{C}_{T}^{-\eta / 4, v}}\right)^{2}, \\
\left\|f^{2}(s)\right\|_{-d} \lesssim & \left\|\Phi^{\prime}\right\|_{\star, 2, T}\left\|X^{2}-a\right\|_{-1-d / 4}^{2} \\
& \times \int_{0}^{s} \mathrm{~d} \sigma(s-\sigma)^{-1+d / 2} \sigma^{-(c+z) / 2} \\
\lesssim_{z, c, d} & s^{d / 2-(c+z) / 2}\left\|\Phi^{\prime}\right\|_{\star, 2, T}\left\|X^{2}-a\right\|_{-1-d / 4}
\end{aligned}
$$

and

$$
\begin{aligned}
\left\|f^{3}(s)\right\|_{2 \eta / 3} \lesssim & \left\|\Phi^{\prime}\right\|_{\star, 2, T}\left\|X^{2}-a\right\|_{-1-\eta / 9}^{2} \\
& \times \int_{0}^{s} \mathrm{~d} s(s-\sigma)^{-1+\eta / 9} s^{-(\eta+z) / 2} \\
\lesssim & s^{-(11 \eta+9 z) / 2}\left\|\Phi^{\prime}\right\|_{\star, 2, T}\left\|X^{2}-a\right\|_{-1-\eta / 9}^{2},
\end{aligned}
$$

where $v>0$ depends only on $L$. A similar bound holds for $f^{4}$, which allows us to conclude by Lemma 3.7 that we have

$$
\sup _{(s, t) \in[0, T]^{2}} s^{\frac{z+a}{2}} \frac{\left\|J\left(\Phi^{\prime}, \mathbb{X}\right)(t)-J\left(\Phi^{\prime}, \mathbb{X}\right)(s)\right\|_{a-2 b}}{|t-s|^{b}} \lesssim T^{\theta}\left\|\Phi^{\prime}\right\|_{\star, 2, T}\left\|X^{\diamond 2}\right\|_{-1-\rho}^{2},
$$

for some $\rho>0, \theta>0$ and $\eta, c, d>0$ small enough and $z \in(1 / 2,2 / 3)$.

We are now able to give the meaning of $I\left(\Phi \diamond X^{\diamond 2}\right)$ for $\Phi \in \mathcal{D}_{\mathbb{X}}^{L}$. 
COROLlary 3.9. Assume that $\mathbb{X} \in \mathcal{X}$ and let $\Phi \in \mathcal{D}_{\mathbb{X}}^{L}$ then for $z \in(1 / 2,2 / 3)$ and for a suitable choice of $L$ the term $I\left(\Phi \diamond X^{\diamond 2}\right)[\Phi, \mathbb{X}]$ is defined via the following expansion:

$$
\begin{aligned}
I\left(\Phi \diamond X^{\diamond 2}\right)[\Phi, \mathbb{X}]:= & B_{<}\left(\Phi, X^{\diamond 2}\right)+B_{>}\left(\Phi, X^{\diamond 2}\right)+I\left(\left(I\left(X^{\diamond 3}\right) \circ X^{\diamond 2}\right)^{\diamond}\right) \\
& +3 J\left(\Phi^{\prime}, \mathbb{X}\right)+B_{0}\left(\Phi^{\sharp}, X^{\diamond 2}\right) .
\end{aligned}
$$

We have the following bound:

$$
\left\|B_{0}\left(\Phi^{\sharp}, X^{\diamond 2}\right)\right\|_{\star, 1, T}+\left\|B_{>}\left(\Phi, X^{\diamond 2}\right)\right\|_{\star, 1, T} \lesssim T^{\theta}\|\Phi\|_{\star, T}\left\|X^{\diamond 2}\right\|_{C_{T}^{-1-\rho}},
$$

for some $\theta, \rho>0$ being nonnegative constants depending on $L$ and $z$. Moreover, if $a, b \in \mathbb{R}, X \in C_{T}^{1}\left(\mathbb{T}^{3}\right)$ and $\varphi \in C^{\infty}([0, T])$, we have

$$
I\left(\Phi \diamond X^{\diamond 2}\right)\left[\Phi, R_{a, b}^{\varphi} \mathbf{X}\right]=I\left(\Phi\left(X^{2}-a\right)\right)+3 b I\left(X+\Phi^{\prime}\right),
$$

for every $\Phi \in \mathcal{D}_{R_{a, b}^{\varphi} \mathbf{x}}$.

PROOF. We remark that all the terms in the definition of $I\left(\Phi \diamond X^{\diamond 2}\right)$ are well defined due to Proposition 3.8 and the definition of the paraproduct. We also notice that

$$
\begin{aligned}
\left\|B_{0}\left(\Phi^{\sharp}, X^{\diamond 2}\right)(t)\right\|_{1+\delta} & \\
& \lesssim \int_{0}^{t} \mathrm{~d} s(t-s)^{-(1+\delta / 2) / 2}\left\|\Phi_{s}^{\sharp}\right\|_{1+\delta}\left\|X^{\diamond 2}\right\|_{-1-\delta / 2} \\
& \lesssim\left\|\Phi^{\sharp}\right\|_{\star, 1, T}\left\|X^{\diamond 2}\right\|_{C_{T}^{-1-\delta / 2}} \int_{0}^{t} \mathrm{~d} s(t-s)^{-(1+\delta / 2) / 2} s^{-(1+\delta+z) / 2} \\
& \lesssim s^{-(3 / 2 \delta+z) / 2}\left\|\Phi^{\sharp}\right\|_{\star, 1, T}\left\|X^{\diamond 2}\right\|_{C_{T}^{-1-\delta / 2}},
\end{aligned}
$$

which gives easily

$$
\sup _{t \in[0, T]} t^{(1+\delta+z) / 2}\left\|B_{0}\left(\Phi^{\sharp}, X^{\diamond 2}\right)(t)\right\|_{1+\delta} \lesssim T^{1 / 2-\delta}\left\|\Phi^{\sharp}\right\|_{\star, 1, T}\left\|X^{\diamond 2}\right\|_{C_{T}^{-1-\delta / 2},}
$$

for $\delta<1 / 2$. By a similar computation, we obtain that there exists $\theta>0$ depending on $L$ and $z$ such that

$$
\begin{aligned}
& \sup _{t \in[0, T]} t^{(1 / 2+\gamma+z) / 2}\left\|B_{0}\left(\Phi^{\sharp}, X^{\diamond 2}\right)(t)\right\|_{1 / 2+\gamma}+\sup _{t \in[0, T]} t^{(\kappa+z) / 2}\left\|B_{0}\left(\Phi^{\sharp}, X^{\diamond 2}\right)(t)\right\|_{\kappa} \\
& \quad \lesssim T^{\theta}\left\|\Phi^{\sharp}\right\|_{\star, 1, T}\left\|X^{\diamond 2}\right\|_{C_{T}^{-1-\delta / 2} .}
\end{aligned}
$$

To obtain the needed bound for this term, we still need to estimate the Hölder-type norm. We remark that

$$
\left\|\Phi_{s}^{\sharp} \circ X_{s}^{\diamond 2}\right\|_{\delta / 2} \lesssim s^{-(1+\delta+z) / 2}\left\|\Phi_{s}^{\sharp}\right\|_{1+\delta}\left\|X_{s}^{\diamond 2}\right\|_{-1-\delta / 2}
$$


and as usual we decompose the norm in the following way:

$$
B_{0}\left(\Phi_{t}^{\sharp}, X^{\diamond 2}\right)(t)-B_{0}\left(\Phi_{s}^{\sharp}, X_{s}^{\diamond 3}\right)=I_{s t}^{1}+I_{s t}^{2},
$$

where

$$
I_{s t}^{1}=\left(P_{t-s}-1\right) \int_{0}^{t} \mathrm{~d} u P_{t-u}\left(\Phi_{u}^{\#} \circ X_{u}^{\diamond 2}\right) \quad \text { and } \quad I_{s t}^{2}=\int_{s}^{t} \mathrm{~d} u P_{t-u}\left(\Phi_{u}^{\sharp} \circ X_{u}^{\diamond 2}\right) .
$$

A straightforward computation gives

$$
\begin{aligned}
\left\|I_{s t}^{1}\right\|_{a-2 b} & \lesssim\left\|\Phi^{\sharp}\right\|_{\star, 1, T}\left\|X^{\diamond 2}\right\|_{C_{T}^{1-\delta / 2}|t-s|^{b} \int_{0}^{t} \mathrm{~d} u(t-u)^{-(a-\delta / 2) / 2} u^{-(1+\delta+z) / 2}} \\
& \lesssim T^{(1-a-\delta / 2-z) / 2}|t-s|^{b}\left\|\Phi^{\sharp}\right\|_{\star, 1, T}\left\|X^{\diamond 2}\right\|_{C_{T}^{1-\delta / 2} .}
\end{aligned}
$$

For $I^{2}$, we use the Hölder inequality, which gives

$$
\begin{aligned}
\left\|I_{s t}^{2}\right\|_{a-2 b} & \lesssim|t-s|^{b}\left\|\Phi^{\sharp}\right\|_{\star, 1, T}\left\|X^{\diamond 2}\right\|_{C_{T}^{1-\delta / 2}}\left(\int_{s}^{t} \mathrm{~d} u(t-u)^{-\frac{a-2 b-\delta / 2}{2(1-b)}} u^{-\frac{1+\delta+z}{2(1-b)}}\right)^{1-b} \\
& \lesssim T^{(1-a-\delta / 2-z) / 2}|t-s|^{b}\left\|\Phi^{\sharp}\right\|_{\star, 1, T}\left\|X^{\diamond 2}\right\|_{C_{T}^{1-\delta / 2}},
\end{aligned}
$$

for $a, \delta>0$ small enough and $z<1$. We have obtained that

$$
\left\|B_{0}\left(\Phi^{\sharp}, X^{\diamond 2}\right)\right\|_{\star, 1, T} \lesssim T^{\theta}\left\|\Phi^{\sharp}\right\|_{\star, 1, T}\left\|X^{\diamond 2}\right\|_{C_{T}^{-1-\delta / 2}},
$$

for some $\theta>0$ depending on $L$ and $z$. The bound for the term $B_{>}\left(\Phi, X^{\diamond 2}\right)$ is obtained by a similar argument and this completes the proof.

REMARK 3.10. When there is no ambiguity, we use the notation $I\left(\Phi \diamond X^{\diamond 2}\right)$ instead of $I\left(\Phi \diamond X^{\diamond 2}\right)[\Phi, \mathbb{X}]$.

3.3. Fixed-point procedure. Using the analysis of $I\left(\Phi \diamond X^{\diamond 2}\right)$ and $I\left(\Phi^{2} \diamond X\right)$ developed in the previous section, we can now show that the equation

$$
\Phi=I\left(X^{\diamond 3}\right)+3 I\left(\Phi \diamond X^{\diamond 2}\right)+3 I\left(\Phi^{2} \diamond X\right)+I\left(\Phi^{3}\right)+\Psi
$$

admits a unique solution $\Phi \in \mathcal{D}_{\mathbb{X}}^{L}$ for a suitable choice of $L$ and $z \in(1 / 2,2 / 3)$ via the fixed-point method. We also show that if $u^{\varepsilon}$ is the solution of the regularized equation and $\Phi^{\varepsilon}$ is such that $u^{\varepsilon}=X^{\varepsilon}+\Phi^{\varepsilon}$ then $d\left(\Phi^{\varepsilon}, \Phi\right)$ goes to 0 as $\varepsilon$. Hence, by the convergence of $X^{\varepsilon}$ to $X$, we have the convergence of $u^{\varepsilon}$ to $u=\Phi+X$. Let us begin by giving our fixed-point result.

THEOREM 3.11. Assume that $\mathbb{X} \in \mathcal{X}, u^{0} \in \mathcal{C}^{-z}\left(\mathbb{T}^{3}\right)$ with $z \in(1 / 2,2 / 3)$ and $L$ is such that the bounds of Propositions 3.6 and 3.8 are satisfied. Let $\left(\Phi, \Phi^{\prime}\right) \in$ $\mathcal{D}_{\mathbb{X}}^{L}$ and $\Psi=P u^{0}$. We define the application $\Gamma: \mathcal{D}_{\mathbb{X}, T}^{L} \rightarrow C_{T}^{-z}\left(\mathbb{T}^{3}\right)$ by

$$
\Gamma\left(\Phi, \Phi^{\prime}\right)=I\left(X^{\diamond 3}\right)+3 I\left(\Phi \diamond X^{\diamond 2}\right)+3 I\left(\Phi^{2} \diamond X\right)+I\left(\Phi^{3}\right)+\Psi,
$$


where $I\left(\Phi \diamond X^{\diamond 2}\right)$ and $I\left(\Phi^{2} X\right)$ are given by Corollary 3.9 and Proposition 3.6. Then $(\Gamma(\Phi), \Phi) \in \mathcal{D}_{\mathbb{X}}^{L}$ for a suitable choice of $L$ and it satisfies the following bound:

$$
\|\Gamma(\Phi)\|_{\star, T} \lesssim\left(T^{\theta}\|\Phi\|_{\star, L, T}+1\right)^{3}\left(1+\|\mathbb{X}\|_{T, K}+\left\|u^{0}\right\|_{-z}\right)^{3} .
$$

Moreover for $\Phi_{1}, \Phi_{2} \in \mathcal{D}_{\mathbb{X}}^{L}$ the following bound holds:

$$
\begin{aligned}
d_{T, L}( & \left.\Gamma\left(\Phi_{1}\right), \Gamma\left(\Phi_{2}\right)\right) \\
\lesssim & T^{\theta} d_{T, L}\left(\Phi_{1}, \Phi_{2}\right)\left(\left\|\Phi_{1}\right\|_{\star, L, T}+\left\|\Phi_{2}\right\|_{\star, L, T}+1\right)^{2} \\
& \times\left(1+\|\mathbb{X}\|_{T, K}+\left\|u^{0}\right\|_{-z}\right)^{3},
\end{aligned}
$$

for some $\theta>0$ and $K \in[0,1]^{8}$ depending on $L$ and $z$. We can conclude that for this choice of $L$ there exists $T>0$ and a unique $\Phi \in \mathcal{D}_{\mathbb{X}, T}^{L}$ such that

$$
\Phi=\Gamma(\Phi)=I\left(X^{\diamond 3}\right)+3 I\left(\Phi^{2} \diamond X^{\diamond 2}\right)+3 I\left(\Phi^{2} \diamond X\right)+I\left(\Phi^{3}\right)+\Psi .
$$

ProOF. By Corollary 3.9 and Proposition 3.6, we see that $\Gamma(\Phi)$ has the needed algebraic structure of the controlled distribution. More precisely,

$$
\begin{aligned}
\Gamma(\Phi)^{\prime} & =3 \Phi, \\
\Gamma(\Phi)^{\sharp} & =3 B_{>}\left(\Phi, X^{\diamond 2}\right)+X^{\diamond}\left(\Phi^{\prime}\right)+3 B_{0}\left(\Phi^{\sharp}, X^{\diamond 2}\right)+3 I\left(\Phi^{2} X\right)+I\left(\Phi^{3}\right)+\Psi
\end{aligned}
$$

and $\Gamma(\Phi) \in C_{T}^{-z}$. To show that $\Gamma(\Phi) \in \mathcal{D}_{\mathbb{X}}^{L}$ and to obtain the first bound, it remains to estimate $\|\Phi\|_{\star, 2, L, T}$ and $\left\|\Gamma(\Phi)^{\sharp}\right\|_{\star, 1, L, T}$. A straightforward computation gives

$$
\begin{aligned}
&\left\|\Phi_{t}\right\|_{\eta} \lesssim\left\|I\left(X^{\diamond 3}\right)(t)\right\|_{\eta}+\left\|B_{<}\left(\Phi^{\prime}, X^{\diamond 2}\right)(t)\right\|_{\eta}+\left\|\Phi_{t}^{\sharp}\right\|_{\eta} \\
& \lesssim\left\|I\left(X^{\diamond 3}\right)\right\|_{\eta}+\left\|\Phi^{\prime}\right\|_{\star, 2, T}\left\|X^{\diamond 2}\right\|_{-1-\eta} \\
& \quad \times \int_{0}^{t} \mathrm{~d} s(t-s)^{-(1+2 \eta / 2) / 2} s^{-(\eta+z) / 2}+t^{-(\kappa+z)}\left\|\Phi^{\sharp}\right\|_{\star, 1, T} \\
& \lesssim\left(\|\Phi\|_{\star, L, T}+1\right)\left(\left\|X^{\diamond 2}\right\|_{-1-\eta}+\left\|I\left(X^{\diamond 3}\right)\right\|_{\eta}+1\right) \\
& \times t^{\min (1 / 2-(3 \eta+z) / 2,-(\kappa+z) / 2)} .
\end{aligned}
$$

Hence, for $0<\eta<\kappa$ and $\eta<1 / 2$ and $z \in(1 / 2,2 / 3)$ small enough, we have that

$$
\sup _{t \in[0, T]} t^{(\eta+z) / 2}\|\Phi\|_{\eta} \lesssim T^{\kappa-\eta}\left(\|\Phi\|_{\star, T}+1\right)\left(\left\|X^{\diamond 2}\right\|_{C_{T}^{-1-\eta}}+\left\|I\left(X^{\diamond 3}\right)\right\|_{C_{T}^{\eta}}+1\right) .
$$

We focus on the explosive Hölder-type norm for this term. Indeed a quick computation gives

$$
\begin{aligned}
\left\|\Phi_{t}-\Phi_{s}\right\|_{c-2 d} \lesssim & \left\|I\left(X^{\diamond 3}\right)(t)-I\left(X^{\diamond 3}\right)(s)\right\|_{c-2 d} \\
& +\left\|B_{<}\left(\Phi^{\prime}, X^{\diamond 2}\right)(t)-B_{<}\left(\Phi^{\prime}, X^{\diamond 2}\right)(s)\right\|_{c-2 d} \\
& +\left\|\Phi_{t}^{\sharp}-\Phi_{s}^{\sharp}\right\|_{c-2 d} .
\end{aligned}
$$


Let us estimate the first term on the right-hand side. Using the regularity for $I\left(X^{\diamond 3}\right)$, we obtain that for $d>0$ small enough and $c<1 / 2$,

$$
\left\|I\left(X^{\diamond 3}\right)(t)-I\left(X^{\diamond 3}\right)(s)\right\|_{c-2 d} \lesssim|t-s|^{d}\left\|I\left(X^{\diamond 3}\right)\right\|_{d, c-2 d} \cdot
$$

We notice that the increment appearing in second term has the following representation:

$$
B_{<}\left(\Phi^{\prime}, X^{\diamond 2}\right)=I(f),
$$

where $f=\pi_{<}\left(\Phi^{\prime}, X^{\diamond 2}\right)$. To treat this term, it is sufficient to notice that

$$
\left\|f_{t}\right\|_{-1-\delta} \lesssim\left\|\Phi_{t}^{\prime}\right\|_{\eta}\left\|X^{\diamond 2}\right\|_{-1-\delta} \lesssim t^{-(\eta+z) / 2}\|\Phi\|_{\star, L, T}\left\|X^{\diamond 2}\right\|_{-1-\delta}
$$

and we have

$$
\begin{aligned}
& \left\|B_{<}\left(\Phi^{\prime}, X^{\diamond 2}\right)(t)-B_{<}\left(\Phi^{\prime}, X^{\diamond 2}\right)(s)\right\|_{c-2 d} \\
& \quad \lesssim T^{\theta}|t-s|^{d} t^{-(c+z) / 2}\|\Phi\|_{\star, L, T}\left\|X^{\diamond 2}\right\|_{-1-\delta},
\end{aligned}
$$

for some $\theta>0$ and $c, \delta>0$. For the last term, we use the fact that

$$
\begin{aligned}
\left\|\Phi_{t}^{\sharp}-\Phi_{s}^{\sharp}\right\|_{c-2 d} & \lesssim|t-s|^{b} t^{-(a+z) / 2}\|\Phi\|_{\star, T} \\
& \lesssim T^{b-d+a-c}|t-s|^{d} t^{-(c+z) / 2}\|\Phi\|_{\star, L, T},
\end{aligned}
$$

for $c-2 d<a-2 b, d<b$ and then $c<a$ which gives

$$
\begin{aligned}
& \sup _{s, t \in[0, T]} s^{-(c+z) / 2} \frac{\left\|\Phi_{t}-\Phi_{s}\right\|_{c-2 d}}{|t-s|^{d}} \\
& \quad \lesssim T^{\theta}\left(1+\left\|I\left(X^{\diamond 3}\right)\right\|_{d, c-2 d}+\left\|X^{\diamond 2}\right\|_{-1-\delta}\right)\left(1+\|\Phi\|_{\star, L, T}\right) .
\end{aligned}
$$

Hence the following bound holds:

$$
\left\|\Gamma(\Phi)^{\prime}\right\|_{\star, 2, L, T} \lesssim T^{\theta}\left(1+\left\|I\left(X^{\diamond 3}\right)\right\|_{d, c-2 d}+\left\|X^{\diamond 2}\right\|_{-1-\delta}\right)\left(1+\|\Phi\|_{\star, T}\right) .
$$

We need to estimate the remaining term $\Gamma(\Phi)^{\sharp}$. Due to Propositions 3.6, 3.8 and Corollary 3.9, it only remains to estimate the following terms $I\left(\Phi^{3}\right)$ and $\Psi$. In fact, an easy computation gives

$$
\|\Psi\|_{\star, 1, L, T} \lesssim\left\|u^{0}\right\|_{-z} .
$$

Let us focus on the term $I\left(\Phi^{3}\right)$. We notice that

$$
\left\|I\left(\Phi^{3}\right)(t)\right\|_{1+\delta} \lesssim \int_{0}^{t} \mathrm{~d} s(t-s)^{-(1+\delta-\eta) / 2} s^{-3 / 2(\eta+z)}\|\Phi\|_{\star, T}^{3}\left(\left\|X^{\diamond 2}\right\|_{-1-\rho}+1\right)^{3},
$$

for $\delta, \kappa>0$ small enough and $z<2 / 3$. Hence we obtain the existence of some $\theta>0$ such that

$$
\sup _{t \in[0, T]} t^{(1+\delta+z) / 2}\left\|I\left(\Phi^{3}\right)(t)\right\|_{1+\delta} \lesssim T^{\theta}\|\Phi\|_{\star, T} .
$$


A similar argument gives

$$
\begin{aligned}
& \sup _{t \in[0, T]} t^{(1 / 2+\gamma+z) / 2}\left\|I\left(\Phi^{3}\right)(t)\right\|_{1 / 2+\gamma}+\sup _{t \in[0, T]} t^{(\kappa+z) / 2}\left\|I\left(\Phi^{3}\right)(t)\right\|_{\kappa} \\
& \quad \lesssim T^{\theta}\|\Phi\|_{\star, L, T}^{3}\left(1+\left\|X^{\diamond 2}\right\|_{-1-\rho}\right)^{3} .
\end{aligned}
$$

Let us remark that

$$
\left\|\Phi_{t}^{3}\right\|_{\eta} \lesssim t^{-3(\eta+z) / 2}\|\Phi\|_{\star, L, T}^{3}\left(1+\left\|X^{\diamond 2}\right\|_{-1-\rho}\right)^{3} .
$$

As usual, to deal with the Hölder norms, we begin by writing the following decomposition:

$$
\left\|I\left(\Phi^{3}\right)(t)-I\left(\Phi^{3}\right)(s)\right\|_{c-2 d} \lesssim I_{s t}^{1}+I_{s t}^{2}
$$

where

$$
I_{s t}^{1}=\left(P_{t-s}-1\right) \int_{0}^{s} \mathrm{~d} u P_{s-u} \Phi_{u}^{3} \quad \text { and } \quad I_{s t}^{2}=\int_{s}^{t} \mathrm{~d} u P_{t-u} \Phi_{u}^{3}
$$

For $I^{1}$, it suffices to observe that

$$
\begin{aligned}
\left\|I_{s t}^{1}\right\|_{c-2 d} & \lesssim|t-s|^{d} \int_{0}^{s} \mathrm{~d} u(s-u)^{-(c-\eta) / 2} u^{-3 / 2(z+\eta)}\|\Phi\|_{\star, T}^{3}\left(1+\left\|X^{\diamond 2}\right\|_{-1-\rho}\right)^{3} \\
& \lesssim T^{1-(c-\eta)-3 / 2(z+\eta)}|t-s|^{d}\|\Phi\|_{\star, L, T}^{3}\left(1+\left\|X^{\diamond 2}\right\|_{-1-\rho}\right)^{3},
\end{aligned}
$$

for $\eta, c>0$ small enough, $z<2 / 3$. To obtain the bound for the second term, we use the Hölder inequality and we have

$$
\begin{aligned}
\left\|I_{s t}^{2}\right\|_{c-2 d} & \lesssim|t-s|^{d}\left(\int_{s}^{t} \mathrm{~d} u\left\|P_{t-u} \Phi_{u}^{3}\right\|_{c-2 d}^{1 /(1-d)}\right)^{1-d} \\
& \lesssim|t-s|^{d}\left(\int_{s}^{t} \mathrm{~d} u(t-u)^{-\frac{c-2 d-\eta}{2-2 d}} u^{-\frac{3(z+\eta)}{(2-2 d)}}\right)^{1-d}\|\Phi\|_{\star, T}^{3}\left(1+\left\|X^{\diamond 2}\right\|_{-1-\rho}\right)^{3} \\
& \lesssim|t-s|^{d} T^{1-(c-2 \eta+3 z) / 2}\|\Phi\|_{\star, T}^{3}\left(1+\left\|X^{\diamond 2}\right\|_{-1-\rho}\right)^{3},
\end{aligned}
$$

for $c, \eta, d>0$ small enough and $z<2 / 3$. We can conclude that there exists $\theta>0$ such that

$$
\sup _{s, t}(z+c) / 2 \frac{\left\|I\left(\Phi^{3}\right)(t)-I\left(\Phi^{3}\right)(s)\right\|_{c-2 d}}{|t-s|^{d}} \lesssim T^{\theta}\|\Phi\|_{\star, T}^{3}\left(1+\left\|X^{\diamond 2}\right\|_{-1-\rho}\right)^{3}
$$

and we obtain all needed bounds for the remaining term. Hence

$$
\left\|\Gamma(\Phi)^{\sharp}\right\|_{\star, 2, L, T} \lesssim\left(T^{\theta}\|\Phi\|_{\star, T}+1\right)^{3}\left(1+\|\mathbb{X}\|_{T, K}+\left\|u^{0}\right\|_{-z}\right)^{3},
$$

for some $K \in[0,1]^{4}$ depending on $L$, which gives the first bound (3.4). The second estimate (3.5) is obtained in the same manner. 
Due to the bound (3.4), for $T_{1}>T>0$ small enough, there exists a constant $R_{T}>0$ such that $B_{R_{T}}:=\left\{\Phi \in \mathcal{D}_{\mathbb{X}, T}^{L} ;\|\Phi\|_{\star, T} \leq R_{T}\right\}$ is invariant by the map $\Gamma$. The bound (3.5) shows that $\Gamma$ is a contraction on $B_{R_{T_{2}}}$ for $0<T_{2}<T_{1}$ small enough. By the usual fixed-point theorem, there exists $\Phi \in \mathcal{D}_{\mathbb{X}, T_{2}}^{L}$ such that $\Gamma(\Phi)=\Phi$. The uniqueness is obtained by a standard argument.

A quick adaptation of the last proof gives a better result (see, e.g., [7] and the continuity result theorem). In fact, the flow is continuous with respect to the rough distribution $\mathbb{X}$ and with respect to the initial condition $\psi$ (or $u^{0}$ ).

PROPOSITION 3.12. Let $\mathbb{X}$ and $\mathbb{Y}$ be two rough distributions such that $\|X\|_{T, K},\|Y\|_{T, K} \leq R, z \in(-2 / 3,-1 / 2), u_{X}^{0}$ and $u_{Y}^{0}$ two initial conditions and $\Phi^{X} \in \mathcal{D}_{T^{X}, X}^{L}$ and $\Phi^{Y} \in \mathcal{D}_{T^{Y}, Y}^{L}$ the two unique solutions of the equations associating to $\mathbb{X}$ and $\mathbb{Y}$, and $T_{X}$ and $T_{Y}$ their respective living times. For $T^{\star}=\inf \left\{T_{X}, T_{Y}\right\}$, the following bound holds:

$$
\left\|\Phi^{X}-\Phi^{Y}\right\|_{C\left([0, T], \mathcal{C}^{-z}\left(\mathbb{T}^{3}\right)\right)} \lesssim d_{T, L}\left(\Phi^{X}, \Phi^{Y}\right) \lesssim_{R} \mathbf{d}_{T, K}(\mathbb{X}, \mathbb{Y})+\left\|u_{X}^{0}-u_{Y}^{0}\right\|_{-z},
$$

for every $T \leq T^{\star}$, where $d$ is defined in Definition 3.3 and $\mathbf{d}$ is defined in Definition 2.15 .

Hence, using this result and combining it with the convergence Theorem 4.3, we have this second corollary, where the convergence of the approximated equation is proved.

COROLlaRY 3.13. Let $z \in(1 / 2,2 / 3), u^{0} \in \mathcal{C}^{-z}$ and denote by $u^{\varepsilon}$ the unique solution (with life times $T^{\varepsilon}$ ) of the equation

$$
\partial_{t} u^{\varepsilon}=\Delta u^{\varepsilon}-\left(u^{\varepsilon}\right)^{3}+C^{\varepsilon} u^{\varepsilon}+\xi^{\varepsilon},
$$

where $\xi^{\varepsilon}$ is a mollification of the space-time white noise $\xi$ and $C^{\varepsilon}=3\left(C_{1}^{\varepsilon}-3 C_{2}^{\varepsilon}\right)$ where $C_{1}^{\varepsilon}$ and $C_{2}^{\varepsilon}$ are the constants given in Definition 4.2. Let us introduce $u=X+\Phi$ where $\Phi$ is the local solution with life-time $T>0$ for the fixed-point equation given in Theorem 3.11. Then we have the following convergence result:

$$
\mathbb{P}\left(d_{T^{\star}, L}\left(\Phi^{\varepsilon}, \Phi\right)>\lambda\right) \longrightarrow_{\varepsilon \rightarrow 0} 0,
$$

for all $\lambda>0$ with $T^{\star}=\inf \left(T, T^{\varepsilon}\right)$ and $\Phi^{\varepsilon}=u^{\varepsilon}-X^{\varepsilon} \in \mathcal{D}_{X^{\varepsilon}, T}^{L}$.

4. Renormalization and construction of the rough distribution. To end the proof of existence and uniqueness for the renormalized equation, we need to prove that the O.U. process associated to the white noise can be extended to a rough distribution of $\mathcal{X}$ (see Definition 2.15). As explained above, to define the appropriate 
process we proceed by regularization and renormalization. Let us take a $a$ smooth radial function $f$ with compact support and such that $f(0)=1$. We regularize $X$ in the following way:

$$
X_{t}^{\varepsilon}=\sum_{k \neq 0} f(\varepsilon k) \hat{X}_{t}(k) e_{k}
$$

and we show that we can choose two divergent constants $C_{1}^{\varepsilon}, C_{2}^{\varepsilon} \in \mathbb{R}^{+}$and a smooth function $\varphi^{\varepsilon}$ such that $R_{C_{1}^{\varepsilon}, C_{2}^{\varepsilon}}^{\varphi^{\varepsilon}} X^{\varepsilon}:=\mathbb{X}^{\varepsilon}$ converges in $\mathcal{X}$. As it has been noticed in the previous sections, without a renormalization procedure there is no finite limit for such a process.

NOTATION 4.1. Let $k_{1}, \ldots, k_{n} \in \mathbb{Z}^{3}$ we denote by $k_{1, \ldots, n}=\sum_{i=1}^{n} k_{i}$, and for a function $f$ we denote by $\delta f$ the increment of the function given by $\delta f_{s t}=f_{t}-f_{s}$.

DEFINITION 4.2. Let

$$
C_{1}^{\varepsilon}=\mathbb{E}\left[\left(X^{\varepsilon}\right)^{2}\right]
$$

and

$$
C_{2}^{\varepsilon}=2 \sum_{k_{1} \neq 0, k_{2} \neq 0} \frac{\left|f\left(\varepsilon k_{1}\right)\right|^{2}\left|f\left(\varepsilon k_{2}\right)\right|^{2}}{\left|k_{1}\right|^{2}\left|k_{2}\right|^{2}\left(\left|k_{1}\right|^{2}+\left|k_{2}\right|^{2}+\left|k_{1,2}\right|^{2}\right)} .
$$

Notice that thanks to the definition of the Littlewood-Paley blocs, we can also choose to write $C_{2}^{\varepsilon}$ as

$$
\begin{aligned}
C_{2}^{\varepsilon}= & 2 \sum_{|i-j| \leq 1} \sum_{k_{1} \neq 0, k_{2} \neq 0} \theta\left(2^{-i}\left|k_{1,2}\right|\right) \theta\left(2^{-j}\left|k_{1,2}\right|\right) \\
& \times \frac{f\left(\varepsilon k_{1}\right) f\left(\varepsilon k_{2}\right)}{\left|k_{1}\right|^{2}\left|k_{2}\right|^{2}\left(\left|k_{1}\right|^{2}+\left|k_{2}\right|^{2}+\left|k_{1,2}\right|^{2}\right)} .
\end{aligned}
$$

Let us define the following renormalized quantities:

$$
\begin{aligned}
\left(X^{\varepsilon}\right)^{\diamond 2} & :=\left(X^{\varepsilon}\right)^{2}-C_{1}^{\varepsilon}, \\
I\left(\left(X^{\varepsilon}\right)^{\diamond 3}\right) & :=I\left(\left(X^{\varepsilon}\right)^{3}-3 C_{1}^{\varepsilon} X^{\varepsilon}\right), \\
\left(I\left(\left(X^{\varepsilon}\right)^{\diamond 2}\right) \circ\left(X^{\varepsilon}\right)^{\diamond 2}\right)^{\diamond} & =I\left(\left(X^{\varepsilon}\right)^{\diamond 2}\right) \circ\left(X^{\varepsilon}\right)^{\diamond 2}-C_{2}^{\varepsilon}
\end{aligned}
$$

and

$$
\left(I\left(\left(X^{\varepsilon}\right)^{\diamond 3}\right) \circ\left(X^{\varepsilon}\right)^{\diamond 2}\right)^{\diamond}=I\left(\left(X^{\varepsilon}\right)^{\diamond 3}\right) \circ\left(X^{\varepsilon}\right)^{\diamond 2}-3 C_{2}^{\varepsilon} X^{\varepsilon} .
$$

Then the following theorem holds. 
THEOREM 4.3. For $T>0$, there exists a deterministic sequence of functions $\varphi^{\varepsilon}:[0, T] \rightarrow \mathbb{R}$, a deterministic distribution $\varphi:[0, T] \rightarrow \mathbb{R}$ such that for all $\delta, \delta^{\prime}, \nu, \rho>0$ small enough with $v>\rho$ we have

$$
\|\varphi\|_{\nu, \rho, T}=\sup _{t} t^{\nu}\left|\varphi_{t}\right|+\sup _{0 \leq s<t \leq T} s^{\nu} \frac{\left|\varphi_{t}-\varphi_{s}\right|}{|t-s|^{\rho}}<+\infty
$$

and the sequence $\varphi^{\varepsilon}$ converges to $\varphi$ according to that norm, that is,

$$
\left\|\varphi^{\varepsilon}-\varphi\right\|_{1, \star, T} \rightarrow 0 \text {. }
$$

Furthermore, there exists some stochastic processes

$$
\begin{aligned}
X^{\diamond 2} & \in \mathcal{C}\left([0, T], \mathcal{C}^{-1-\delta}\right), \\
I\left(X^{\diamond 3}\right) & \in \mathcal{C}^{\delta^{\prime}}\left([0, T], \mathcal{C}^{1 / 2-\delta-2 \delta^{\prime}}\right), \\
I\left(X^{\diamond 3}\right) \circ X & \in \mathcal{C}^{\delta^{\prime}}\left([0, T], \mathcal{C}^{-\delta-2 \delta^{\prime}}\right), \\
\left(I\left(X^{\diamond 2}\right) \circ X^{\diamond 2}\right)^{\diamond}-\varphi & \in \mathcal{C}^{\delta^{\prime}}\left([0, T], \mathcal{C}^{-\delta-2 \delta^{\prime}}\right)
\end{aligned}
$$

and

$$
\left(I\left(X^{\diamond 3}\right) \circ X^{\diamond 2}\right)^{\diamond}-3 \varphi X \in \mathcal{C}^{\delta^{\prime}}\left([0, T], \mathcal{C}^{-1 / 2-\delta-2 \delta^{\prime}}\right),
$$

such that each component of the sequence $\mathbb{X}^{\varepsilon}$ converges respectively to the corresponding component of the rough distribution $\mathbb{X}$ in the good topologies, that is, for all $\delta, \delta^{\prime}>0$ small enough, and all $p>1$,

(4.1) $X^{\varepsilon} \rightarrow X \quad$ in $L^{p}\left(\Omega, \mathcal{C}^{\delta^{\prime}}\left([0, T], \mathcal{C}^{-1-\delta-3 \delta^{\prime}-3 / 2 p}\right)\right)$,

$$
\begin{aligned}
& \left(X^{\varepsilon}\right)^{\diamond 2} \rightarrow X^{\diamond 2} \quad \text { in } L^{p}\left(\Omega, \mathcal{C}^{\delta^{\prime}}\left([0, T], \mathcal{C}^{-1-\delta-3 \delta^{\prime}-3 / 2 p}\right)\right), \\
& I\left(\left(X^{\varepsilon}\right)^{\diamond 3}\right) \rightarrow I\left(X^{\diamond 3}\right) \quad \text { in } L^{p}\left(\Omega, \mathcal{C}^{\delta^{\prime}}\left([0, T], \mathcal{C}^{1 / 2-\delta-3 \delta^{\prime}-3 / 2 p}\right)\right),
\end{aligned}
$$

$$
\begin{array}{ll}
I\left(\left(X^{\varepsilon}\right)^{\diamond 3}\right) \circ X^{\varepsilon} \rightarrow I\left(X^{\diamond 3}\right) \circ X & \text { in } L^{p}\left(\Omega, \mathcal{C}^{\delta^{\prime}}\left([0, T], \mathcal{C}^{-\delta-3 \delta^{\prime}-3 / 2 p}\right)\right), \\
\left(I\left(\left(X^{\varepsilon}\right)^{\diamond 2}\right) \circ\left(X^{\varepsilon}\right)^{\diamond 2}\right)^{\diamond}-\varphi^{\varepsilon} & \\
\rightarrow\left(I\left(X^{\diamond 2}\right) \circ X^{\diamond 2}\right)^{\diamond}-\varphi & \text { in } L^{p}\left(\Omega, \mathcal{C}^{\delta^{\prime}}\left([0, T], \mathcal{C}^{-\delta-3 \delta^{\prime}-3 / 2 p}\right)\right)
\end{array}
$$

and

$$
\left(I\left(\left(X^{\varepsilon}\right)^{\diamond 3}\right) \circ\left(X^{\varepsilon}\right)^{\diamond 2}\right)^{\diamond}-3 \varphi^{\varepsilon} X^{\varepsilon} \rightarrow\left(I\left(X^{\diamond 3}\right) \circ X^{\diamond 2}\right)^{\diamond}-3 \varphi X
$$

in $L^{p}\left(\Omega, \mathcal{C}^{\delta^{\prime}}\left([0, T], \mathcal{C}^{-1 / 2-\delta-3 \delta^{\prime}-3 / 2 p}\right)\right)$.

REMARK 4.4. Thanks to the proof below (especially Sections 4.5 and 4.6) we have the following expressions for $\varphi^{\varepsilon}$ and $\varphi$ :

$$
\begin{aligned}
\varphi_{t}^{\varepsilon}= & -\sum_{|i-j| \leq 1} \sum_{k_{1} \neq 0, k_{2} \neq 0} \frac{\left|\theta\left(2^{-i}\left|k_{12}\right|\right)\right|\left|\theta\left(2^{-j}\left|k_{12}\right|\right)\right|\left|f\left(\varepsilon k_{1}\right) f\left(\varepsilon k_{2}\right)\right|}{\left|k_{1}\right|^{2}\left|k_{2}\right|^{2}\left(\left|k_{1}\right|^{2}+\left|k_{2}\right|^{2}+\left|k_{1}+k_{2}\right|^{2}\right)} \\
& \times \exp \left(-t\left(\left|k_{1}\right|^{2}+\left|k_{2}\right|^{2}+\left|k_{1}+k_{2}\right|^{2}\right)\right)
\end{aligned}
$$


and

$$
\begin{aligned}
\varphi_{t}= & -\sum_{|i-j| \leq 1} \sum_{k_{1} \neq 0, k_{2} \neq 0} \frac{\left|\theta\left(2^{-i}\left|k_{12}\right|\right)\right|\left|\theta\left(2^{-j}\left|k_{12}\right|\right)\right|}{\left|k_{1}\right|^{2}\left|k_{2}\right|^{2}\left(\left|k_{1}\right|^{2}+\left|k_{2}\right|^{2}+\left|k_{1}+k_{2}\right|^{2}\right)} \\
& \times \exp \left(-t\left(\left|k_{1}\right|^{2}+\left|k_{2}\right|^{2}+\left|k_{1}+k_{2}\right|^{2}\right)\right) .
\end{aligned}
$$

We split the proof of this theorem according to the various components. We start by the convergence of $X^{\varepsilon}$ to $X$. We also give a full proof for $X^{\diamond 2}$. For the other components, we only give the crucial estimates.

4.1. Convergence to $X$. We start by an easy computation for the convergence of $X^{\varepsilon}$.

ProOF OF (4.1). By a quick computation, we have that

$$
\delta\left(X-X^{\varepsilon}\right)_{s t}=\sum_{k}(f(\varepsilon k)-1) \delta \hat{X}_{s t}(k) e_{k} .
$$

Then

$$
\begin{aligned}
\mathbb{E}\left[\left|\Delta_{q} \delta\left(X-X^{\varepsilon}\right)_{s t}\right|^{2}\right] & =2 \sum_{k \neq 0 ;|k| \sim 2^{q}}|f(\varepsilon k)-1|^{2} \frac{1-e^{-|k|^{2}|t-s|}}{|k|^{2}} \\
& \lesssim_{h, \rho} c(\varepsilon) 2^{q(1+2 h+\rho)}|t-s|^{h},
\end{aligned}
$$

for $h, \rho>0$ small enough, and $c(\varepsilon)=\sum_{k \neq 0}|k|^{-3-\rho}|f(\varepsilon k)-1|^{2}$. The Gaussian hypercontractivity gives for $p>2$,

$$
\begin{aligned}
\mathbb{E}\left[\left\|\Delta_{q} \delta\left(X-X^{\varepsilon}\right)_{s t}\right\|_{L^{p}}^{p}\right] & \lesssim p \int_{\mathbb{T}^{3}} \mathbb{E}\left[\left|\Delta_{q} \delta\left(X-X^{\varepsilon}\right)_{s t}(x)\right|^{2}\right]^{p / 2} \mathrm{~d} x \\
& \lesssim \rho, h c(\varepsilon)^{p}|t-s|^{h p / 2} 2^{q p / 2(2 h+\rho+1)} .
\end{aligned}
$$

We obtain that

$$
\mathbb{E}\left[\left\|\delta\left(X-X^{\varepsilon}\right)_{s t}\right\|_{B_{p, p}^{-1 / 2-\rho-h}}^{p}\right] \lesssim c(\varepsilon)^{p / 2}|t-s|^{h p / 2} .
$$

Using the Besov embedding (Proposition 2.2), we have

$$
\mathbb{E}\left[\left\|\delta\left(X-X^{\varepsilon}\right)_{s t}\right\|_{\mathcal{C}^{-1 / 2-\rho-h-3 / p}}^{p}\right] \lesssim c(\varepsilon)^{p / 2}|t-s|^{h p / 2}
$$

and by the standard Garsia-Rodemich-Rumsey lemma (see [5]) we finally obtain

$$
\mathbb{E}\left[\left\|X-X^{\varepsilon}\right\|_{\mathcal{C}^{h-\theta}\left([0, T], \mathcal{C}^{-1 / 2-h-\rho-3 / p)}\right.}\right] \lesssim c(\varepsilon)^{p},
$$

for all $h>\theta>0, \rho>0$ small enough and $p>2$. Moreover, we have $X_{0}=X_{0}^{\varepsilon}=0$ and by using the fact that $c(\varepsilon) \rightarrow^{\varepsilon \rightarrow 0} 0$, we obtain that

$$
\lim _{\varepsilon \rightarrow 0}\left\|X^{\varepsilon}-X\right\|_{L^{p}\left(\Omega, C_{T}^{\delta^{\prime},-1 / 2-\delta-3 / p}\right)}=0,
$$

for all $0<\delta^{\prime}<\delta / 3$ and $T>0$. 
4.2. Renormalization for $X^{2}$. To prove the theorem for $X^{\diamond 2}$, we first prove the following estimate, and we then use the Garsia-Rodemich-Rumsey lemma to conclude.

PROPOSITION 4.5. Let $p>1, \theta>0$ be small enough, then the following bounds hold:

$$
\sup _{\varepsilon} \mathbb{E}\left[\left\|\Delta_{q} \delta\left(X^{\varepsilon}\right)_{s t}^{\diamond 2}\right\|_{L^{2 p}}^{2 p}\right] \lesssim p, \theta|t-s|^{p \theta} 2^{2 q p(1+2 \theta)}
$$

and

$$
\mathbb{E}\left[\left\|\Delta_{q}\left(\delta\left(X^{\varepsilon}\right)_{s t}^{\diamond 2}-\delta\left(X^{\varepsilon^{\prime}}\right)_{s t}^{\diamond 2}\right)\right\|_{L^{2 p}}^{2 p}\right] \lesssim_{p, \theta} C\left(\varepsilon, \varepsilon^{\prime}\right)^{p}|t-s|^{2 p \theta} 2^{2 q p(1+\theta)},
$$

where $C\left(\varepsilon, \varepsilon^{\prime}\right) \rightarrow 0$ when $\left|\varepsilon-\varepsilon^{\prime}\right| \rightarrow 0$.

PROOF. By a straightforward computation, we have

$$
\begin{aligned}
\operatorname{Var}\left(\Delta_{q}\right. & \left.\left(\left(X_{t}^{\varepsilon}-X_{s}^{\varepsilon}\right) X_{s}^{\varepsilon}\right)\right) \\
= & \sum_{k, k^{\prime} \in \mathbb{Z}^{3}} \theta\left(2^{-q} k\right) \theta\left(2^{-q} k^{\prime}\right) \sum_{k_{12}=k ; k_{12}^{\prime}=k^{\prime}} f\left(\varepsilon k_{1}\right) f\left(\varepsilon k_{2}\right) f\left(\varepsilon k_{1}^{\prime}\right) f\left(\varepsilon k_{2}^{\prime}\right) \\
& \times\left(I_{s t}^{1}+I_{s t}^{2}\right) e_{k} e_{-k^{\prime}},
\end{aligned}
$$

where $\left(e_{k}\right)$ denotes the Fourier basis of $L^{2}\left(\mathbb{T}^{3}\right)$ and

$$
\begin{aligned}
I_{s t}^{1} & =\mathbb{E}\left[\left(\hat{X}_{t}\left(k_{1}\right)-\hat{X}_{s}\left(k_{1}\right)\right)\left(\overline{\hat{X}_{t}\left(k_{1}^{\prime}\right)-\hat{X}_{s}\left(k_{1}^{\prime}\right)}\right)\right] \mathbb{E}\left[\hat{X}_{s}\left(k_{2}\right) \bar{X}_{s}\left(k_{2}^{\prime}\right)\right] \\
& =2 \delta_{k_{1}=k_{1}^{\prime}} \delta_{k_{2}=k_{2}^{\prime}} \frac{1-e^{-\left|k_{1}\right|^{2}|t-s|}}{\left|k_{1}\right|^{2}\left|k_{2}\right|^{2}}
\end{aligned}
$$

and

$$
\begin{aligned}
I_{s t}^{2} & =\mathbb{E}\left[\left(\hat{X}_{t}\left(k_{1}\right)-\hat{X}_{s}\left(k_{1}\right)\right) \overline{\hat{X}_{s}\left(k_{2}^{\prime}\right)}\right] \mathbb{E}\left[\left(\overline{\hat{X}_{t}\left(k_{1}^{\prime}\right)-\hat{X}_{s}\left(k_{1}^{\prime}\right)}\right) \hat{X}_{s}\left(k_{2}\right)\right] \\
& =\delta_{k_{1}=k_{2}^{\prime}} \delta_{k_{1}^{\prime}=k_{2}} \frac{\left(1-e^{-\left|k_{1}\right|^{2}|t-s|}\right)\left(1-e^{-\left|k_{2}\right|^{2}|t-s|}\right)}{\left|k_{1}\right|^{2}\left|k_{2}\right|^{2}} .
\end{aligned}
$$

Injecting these two identities in equation (4.7), we obtain

$$
\begin{aligned}
\operatorname{Var}( & \left.\Delta_{q}\left(\left(X_{t}^{\varepsilon}-X_{s}^{\varepsilon}\right) X_{s}^{\varepsilon}\right)\right) \\
& \lesssim \sum_{\substack{|k| \sim 2^{q} \\
k_{12}=k}} \frac{1-e^{-\left|k_{1}\right|^{2}|t-s|}}{\left|k_{1}\right|^{2}\left|k_{2}\right|^{2}}+\sum_{\substack{|k| \sim 2^{q} \\
k_{12}=k}} \frac{\left(1-e^{-\left|k_{1}\right|^{2}|t-s|}\right)\left(1-e^{-\left|k_{2}\right|^{2}|t-s|}\right)}{\left|k_{1}\right|^{2}\left|k_{2}\right|^{2}} \\
& \lesssim \sum_{\substack{|k| \sim 2^{q} \\
k_{12}=k}} \frac{1-e^{-\left|k_{1}\right|^{2}|t-s|}}{\left|k_{1}\right|^{2}\left|k_{2}\right|^{2}} .
\end{aligned}
$$


We have

$$
\begin{aligned}
& \sum_{\substack{|k| \sim 2^{q} \\
k_{12}=k,\left|k_{1}\right| \leq\left|k_{2}\right|}} \frac{1-e^{-\left|k_{1}\right|^{2}|t-s|}}{\left|k_{1}\right|^{2}\left|k_{2}\right|^{2}} \\
& \quad \lesssim|t-s|^{\theta} \sum_{\substack{k \in \mathbb{Z}^{3} ;|k| \sim 2^{q}, k_{12}=k\\
}}\left|k_{1}\right|^{-2+2 \theta}\left|k_{2}\right|^{-2} \\
& \quad \lesssim|t-s|^{\theta}\left\{\sum_{\substack{|k| \sim 2^{q}, k_{12}=k,\left|k_{1}\right| \leq\left|k_{2}\right|}},\left|k_{1}\right|^{-2+2 \theta}\left|k_{2}\right|^{-2}+\sum_{\substack{|k| \sim 2^{q}, k_{12}=k,\left|k_{1}\right| \geq\left|k_{2}\right|}}\left|k_{1}\right|^{-2+2 \theta}\left|k_{2}\right|^{-2}\right\} \\
& \lesssim|t-s|^{\theta} 2^{2 q(1+2 \theta)}\left(\sum_{k_{1}}\left|k_{1}\right|^{-3-2 \theta}+\sum_{k_{1}}\left|k_{2}\right|^{-3-4 \theta}\right)<+\infty
\end{aligned}
$$

and by the Gaussian hypercontractivity we finally have

$$
\mathbb{E}\left[\left\|\Delta_{q} \delta\left(X^{\varepsilon}\right)_{s t}^{\diamond 2}\right\|_{L^{2 p}}^{2 p}\right]=\int_{\mathbb{T}^{3}}\left(\operatorname{Var}\left(\delta\left(X^{\varepsilon}\right)_{s t}^{\diamond 2}\right)(\xi)\right)^{p} \mathrm{~d} \xi \lesssim|t-s|^{p \theta} 2^{2 q p(1+2 \theta)} .
$$

For the second assertion, we see that the computation of the beginning gives

$$
\operatorname{Var}\left(\left(\Delta_{q}\left(\left(X_{t}^{\varepsilon}-X_{s}^{\varepsilon}\right) X_{s}^{\varepsilon}\right)-\left(X_{t}^{\varepsilon}-X_{s}^{\varepsilon}\right) X_{s}^{\varepsilon}\right)\right) \lesssim|t-s|^{\theta} 2^{2 q(1+3 \theta)} C\left(\varepsilon, \varepsilon^{\prime}\right),
$$

where

$$
\begin{aligned}
C\left(\varepsilon, \varepsilon^{\prime}\right) & =\sum_{k_{12}=k}\left(\left|f\left(\varepsilon k_{1}\right)\right|^{2}\left|f\left(\varepsilon k_{2}\right)\right|^{2}-\left|f\left(\varepsilon^{\prime} k_{1}\right)\right|^{2}\left|f\left(\varepsilon^{\prime} k_{2}\right)\right|^{2}\right)|k|^{-3-\theta}\left|k_{1}\right|^{-3-2 \theta} \\
& \rightarrow{ }^{\left|\varepsilon-\varepsilon^{\prime}\right| \rightarrow 0} 0
\end{aligned}
$$

by the dominated convergence theorem. Once again, the Gaussian hypercontractivity gives us the needed bounds.

Using the Besov embedding (Proposition 2.2) combined with the standard Garsia-Rodemich-Rumsey lemma (see [5]) the following convergence result holds.

Proposition 4.6. Let $\theta, \delta, \rho>0$ be small enough such that $\rho<\theta / 2$ and $p>1$ then the following bound holds:

$$
\mathbb{E}\left[\left\|\left(X^{\varepsilon}\right)^{\diamond 2}-\left(X^{\varepsilon^{\prime}}\right)^{\diamond 2}\right\|_{\mathcal{C}^{\theta / 2-\rho}\left([0, T], \mathcal{C}^{-1-3 /(2 p)-\delta-2 \theta}\right)}^{2 p}\right] \lesssim_{\theta, p, \delta} C\left(\varepsilon, \varepsilon^{\prime}\right)^{p} .
$$

Since $\left(X_{0}^{\varepsilon}\right)^{\diamond 2}=0$ and $\left(X^{\diamond 2}\right)_{0}=0$, the sequence $\left(X^{\varepsilon}\right)^{\diamond 2}$ converges in

$$
L^{2 p}\left(\Omega, C^{\theta / 2-\rho}\left([0, T], \mathcal{C}^{-1-3 /(2 p)-\delta-3 \theta}\right)\right)
$$

to a random field denoted by $X^{\diamond 2}$. 
4.3. Renormalization for $I\left(X^{3}\right)$. As the computations are quite similar, we only prove the equivalent of the $L^{2}$ estimates in Proposition 4.5. Furthermore, we only prove it for a fixed time $t$ and not for an increment.

ProOF OF (4.3). By a simple computation, we have

$$
I\left(\left(X_{t}^{\varepsilon}\right)^{\diamond 3}\right)=\sum_{k \in \mathbb{Z}^{3}}\left(\int_{0}^{t} \mathcal{F}\left(\left(X_{s}^{\varepsilon}\right)^{\diamond 3}\right)(k) e^{-|k|^{2}|t-s|} \mathrm{d} s\right) e_{k} .
$$

Hence

$$
\begin{aligned}
\mathbb{E}\left[\left|\Delta_{q} I\left(\left(X_{t}^{\varepsilon}\right)^{\diamond 3}\right)\right|^{2}\right]= & 6 \sum_{\substack{k \in \mathbb{Z}^{3} \\
k_{123}=k}}\left|\theta\left(2^{-q} k\right)\right|^{2} \prod_{i=1, \ldots, 3} \frac{\left|f\left(\varepsilon k_{i}\right)\right|^{2}}{\left|k_{i}\right|^{2}} \int_{0}^{t} \mathrm{~d} s \\
& \times \int_{0}^{s} \mathrm{~d} \sigma e^{-\left(\left|k_{1}\right|^{2}+\left|k_{2}\right|^{2}+\left|k_{3}\right|^{2}\right)|s-\sigma|-|k|^{2}(|t-s|+|t-\sigma|)} \\
= & \sum_{k}\left|\theta\left(2^{-q} k\right)\right|^{2} \Xi^{\varepsilon, 1}(k),
\end{aligned}
$$

where

$$
\begin{aligned}
\Xi^{\varepsilon, 1}(k)= & \sum_{k_{123}=k, k_{i} \neq 0} \prod_{i=1, \ldots, 3} \frac{\left|f\left(\varepsilon k_{i}\right)\right|^{2}}{\left|k_{i}\right|^{2}} \\
& \times \int_{0}^{t} \mathrm{~d} s \int_{0}^{s} \mathrm{~d} \sigma e^{-\left(\left|k_{1}\right|^{2}+\left|k_{2}\right|^{2}+\left|k_{3}\right|^{2}\right)|s-\sigma|-|k|^{2}(|t-s|+\mid t-\sigma)} \\
\lesssim & \sum_{k_{123}=k} \frac{1}{\left|k_{1}\right|^{2}\left|k_{2}\right|^{2}\left|k_{3}\right|^{2}} \\
& \times \int_{0}^{t} \mathrm{~d} s \int_{0}^{s} \mathrm{~d} \sigma e^{-\left(\left|k_{1}\right|^{2}+\left|k_{2}\right|^{2}+\left|k_{3}\right|^{2}\right)|s-\sigma|-|k|^{2}|t-s|} \\
\lesssim & \frac{1}{|k|^{2-\rho}} \sum_{k_{123}=k} \frac{1}{\left|k_{1}\right|^{4-\rho}\left|k_{2}\right|^{2}\left|k_{3}\right|^{2}} \\
\lesssim & \frac{1}{|k|^{4-4 \rho}}\left(\sum_{k_{2}}\left|k_{2}\right|^{-3-\rho}\right)^{2} .
\end{aligned}
$$

We have used that

$$
\begin{aligned}
\int_{0}^{t} \mathrm{~d} s & \int_{0}^{s} \mathrm{~d} \sigma e^{-\left(\left|k_{1}\right|^{2}+\left|k_{2}\right|^{2}+\left|k_{3}\right|^{2}\right)|s-\sigma|-|k|^{2}(|t-s|+\mid t-\sigma)} \\
& \lesssim T \frac{1}{\left|k_{1}\right|^{2-\rho}|k|^{2-\rho}} \int_{0}^{t} \mathrm{~d} s \int_{0}^{s} \mathrm{~d} \sigma|t-s|^{-1+\rho / 2}|s-\sigma|^{-1+\rho / 2}
\end{aligned}
$$


for $\rho>0$ small enough. Using again the Gaussian hypercontractivity, we have

$$
\mathbb{E}\left[\left\|\Delta_{q} I\left(\left(X_{t}^{\varepsilon}\right)^{\diamond 3}\right)\right\|_{L^{2 p}}^{2 p}\right] \lesssim 2^{-2 p q(1 / 2-\rho)}
$$

and the Besov embedding gives

$$
\sup _{t \in[0, T], \varepsilon} \mathbb{E}\left[\left\|I\left(\left(X_{t}^{\varepsilon}\right)^{\diamond 3}\right)\right\|_{1 / 2-\rho-3 / p}\right]<+\infty .
$$

The same computation gives

$$
\sup _{t \in[0, T]} \mathbb{E}\left[\left\|I\left(\left(X_{t}^{\varepsilon}\right)^{\diamond 3}\right)-I\left(\left(X_{t}^{\varepsilon^{\prime}}\right)^{\diamond 3}\right)\right\|_{1 / 2-\rho-3 / p}^{2 p}\right] \rightarrow^{\left|\varepsilon^{\prime}-\varepsilon\right| \rightarrow 0} 0,
$$

which gives the needed convergence.

4.4. Renormalization for $I\left(X^{\diamond 3}\right) \circ X$. Here, we only prove the $L^{2}$ estimate for the term $I\left(X^{\diamond 3}\right) X$ instead of $I\left(X^{\diamond 3}\right) \circ X$ since the computations in the two cases are essentially similar. We remark that in that case we do not need a renormalization.

PROOF OF (4.4). We have the following representation formula:

$$
\begin{aligned}
& \mathbb{E}\left[\left|\Delta_{q}\left(I\left(X^{\varepsilon}\right)^{\diamond 3} X^{\varepsilon}\right)(t)\right|^{2}\right] \\
& \quad=\sum_{k}\left|\theta\left(2^{-q} k\right)\right|^{2}\left(6 I_{1}^{\varepsilon}(t)(k)+18 I_{2}^{\varepsilon}(t)(k)+18 I_{3}^{\varepsilon}(t)(k)\right),
\end{aligned}
$$

where

$$
\begin{aligned}
I_{1}^{\varepsilon}(t)(k)= & 2 \sum_{k_{1234}=k} \prod_{i=1, \ldots, 4} \frac{\left|f\left(\varepsilon k_{i}\right)\right|^{2}}{\left|k_{i}\right|^{2}} \\
& \times \int_{0}^{t} \mathrm{~d} s \int_{0}^{s} \mathrm{~d} \sigma e^{-\left|k_{123}\right|^{2}(|t-s|+|t-\sigma|)-\left(\left|k_{1}\right|^{2}+\left|k_{2}\right|^{2}+\left|k_{3}\right|^{2}\right)|s-\sigma|}, \\
I_{12}^{\varepsilon}(t)(k)= & \sum_{\substack{k_{1234}=k \\
\max _{i, 1,3}\left|k_{i}\right|=\left|k_{1}\right| \\
\left|k_{123}\right| \geq\left|k_{4}\right|}}\left(\frac{1}{\left|k_{1}\right|\left|k_{2}\right|\left|k_{3}\right|\left|k_{4}\right|}\right)^{2} \\
& \times \int_{0}^{t} \mathrm{~d} s \int_{0}^{s} \mathrm{~d} \sigma e^{-\left|k_{123}\right|^{2}(|t-s|+|t-\sigma|)-\left(\left|k_{1}\right|^{2}+\left|k_{2}\right|^{2}+\left|k_{3}\right|^{2}\right)|s-\sigma|}
\end{aligned}
$$

and

$$
\begin{aligned}
I_{3}^{\varepsilon}(t)(k)= & \sum_{\substack{k_{12}=k \\
k_{3}, k_{4}}} \prod_{i=1}^{4} \frac{\left|f\left(\varepsilon k_{i}\right)\right|^{2}}{\left|k_{i}\right|^{2}} \\
& \times \int_{0}^{t} \mathrm{~d} s \int_{0}^{t} \mathrm{~d} \sigma e^{-\left(\left|k_{1}\right|^{2}+\left|k_{2}\right|^{2}\right)|s-\sigma|-\left(\left|k+k_{3}\right|^{2}+\left|k_{3}\right|^{2}\right)|t-s|-\left(\left|k_{4}\right|^{2}+\left|k+k_{4}\right|^{2}\right)|t-\sigma|} .
\end{aligned}
$$


We have

$$
\begin{aligned}
I_{1}^{\varepsilon}(t)(k) \lesssim & \sum_{\substack{k_{1234}=k \\
\max _{i=1,2,3}\left|k_{i}\right|=\left|k_{1}\right|}} \frac{1}{\left|k_{1}\right|^{2}\left|k_{2}\right|^{2}\left|k_{3}\right|^{2}\left|k_{4}\right|^{2}} \\
& \quad \times \int_{0}^{t} \mathrm{~d} s \int_{0}^{s} \mathrm{~d} \sigma e^{-\left|k_{123}\right|^{2}(|t-s|+|t-\sigma|)-\left(\left|k_{1}\right|^{2}+\left|k_{2}\right|^{2}+\left|k_{3}\right|^{2}\right)|s-\sigma|} \\
\lesssim & I_{11}^{\varepsilon}(t)(k)+I_{12}^{\varepsilon}(t)(k),
\end{aligned}
$$

where

$$
\begin{aligned}
I_{11}^{\varepsilon}(t)(k)= & \sum_{\substack{k_{1234}=k \\
\max _{i=1,2,3}\left|k_{i}\right|=\left|k_{1}\right| \\
\left|k_{123}\right| \leq\left|k_{4}\right|}} \frac{1}{\left|k_{1}\right|^{2}\left|k_{2}\right|^{2}\left|k_{3}\right|^{2}\left|k_{4}\right|^{2}} \\
& \times \int_{0}^{t} \mathrm{~d} s \int_{0}^{s} \mathrm{~d} \sigma e^{-\left|k_{123}\right|^{2}(|t-s|+|t-\sigma|)-\left(\left|k_{1}\right|^{2}+\left|k_{2}\right|^{2}+\left|k_{3}\right|^{2}\right)|s-\sigma|}
\end{aligned}
$$

and

$$
\begin{aligned}
I_{12}^{\varepsilon}(t)(k) \lesssim & \sum_{\substack{k_{1234}=k \\
\max _{i=1,2,3}\left|k_{i}\right|=\left|k_{1}\right| \\
\left|k_{123}\right| \geq\left|k_{4}\right|}} \frac{1}{\left|k_{1}\right|^{2}\left|k_{2}\right|^{2}\left|k_{3}\right|^{2}\left|k_{4}\right|^{2}} \\
& \times \int_{0}^{t} \mathrm{~d} s \int_{0}^{s} \mathrm{~d} \sigma e^{-\left|k_{123}\right|^{2}(|t-s|+|t-\sigma|)-\left(\left|k_{1}\right|^{2}+\left|k_{2}\right|^{2}+\left|k_{3}\right|^{2}\right)|s-\sigma|}
\end{aligned}
$$

Hence

$$
\begin{aligned}
I_{11}^{\varepsilon}(t)(k) & \lesssim \frac{1}{|k|^{2}} \sum_{k_{2}, k_{3}, k_{1}, \max \left|k_{i}\right|=\left|k_{1}\right|} \frac{1}{\left|k_{1}\right|^{4-\rho}\left|k_{2}\right|^{2}\left|k_{3}\right|^{2}\left|k_{123}\right|^{2-\rho}} \\
& \lesssim \frac{1}{|k|^{2}} \sum_{k_{1}, k_{2}, k_{3}} \frac{1}{\left|k_{2}\right|^{3+\rho}\left|k_{3}\right|^{3+\rho}\left|k_{123}\right|^{3+\rho}} \\
& \lesssim|k|^{-2},
\end{aligned}
$$

for $\rho>0$ small enough, which is the needed result for $I_{1,1}^{\varepsilon}$. We can treat the second term by a similar computation. Indeed

$$
I_{12}^{\varepsilon}(t)(k) \lesssim|k|^{-2+\rho} \sum_{k_{2}, k_{3}, k_{4}}\left|k_{2}\right|^{-3-\rho}\left|k_{2}\right|^{-3-\rho}\left|k_{3}\right|^{-3-\rho} \lesssim|k|^{-2+\rho},
$$

for $\rho>0$ small enough. This gives the bound for $I_{1,2}^{\varepsilon}$ and $I_{1}^{\varepsilon}$. More precisely, we have $I_{1}^{\varepsilon}(t)(k) \lesssim|k|^{-2+\rho}$ for $\rho>0$ small enough. Let us focus on the second term 
$I_{2}^{\varepsilon}(t)(k)$. We have

$$
\begin{aligned}
& I_{2}^{\varepsilon}(t)(k) \lesssim \sum_{\substack{k_{12}=k \\
\max _{i=1,2}\left|k_{i}\right|=\left|k_{1}\right| \\
k_{3}, k_{4}}} \frac{1}{\left|k_{1}\right|^{1-\rho}\left|k_{2}\right|^{3+\rho}\left|k_{3}\right|^{2}\left|k_{4}\right|^{2}} \\
& \times \int_{0}^{t} \mathrm{~d} s \int_{0}^{t} \mathrm{~d} \sigma e^{-\left(\left|k-k_{3}\right|^{2}+\left|k_{3}\right|^{2}\right)|t-s|-\left(\left|k_{4}\right|^{2}+\left|k-k_{4}\right|^{2}\right)|t-\sigma|} \\
& \lesssim \rho \frac{1}{|k|^{1-\rho}}\left(\sum_{k_{3}} \frac{1}{\left|k_{3}\right|^{2}} \int_{0}^{t} \mathrm{~d} s e^{-\left(\left|k_{3}\right|^{2}+\left|k-k_{3}\right|^{2}\right)|t-s|}\right)^{2} \\
& \lesssim \rho, T \frac{1}{|k|^{1-\rho}}\left(\sum_{k_{3} \neq 0, k} \frac{1}{\left|k_{3}\right|^{2}\left|k-k_{3}\right|^{2-\rho}}\right)^{2} \lesssim T, \rho \frac{1}{|k|^{3-3 \rho}}
\end{aligned}
$$

and we obtain the bound for $I_{2}^{\varepsilon}$. We notice that

$$
\begin{aligned}
I_{3}^{\varepsilon}(t)(k)= & \sum_{\substack{k_{12}=k \\
k_{3}, k_{4}}} \prod_{i=1}^{4} \frac{\left|f\left(\varepsilon k_{i}\right)\right|^{2}}{\left|k_{i}\right|^{2}} \\
& \times \int_{0}^{t} \mathrm{~d} s \int_{0}^{t} \mathrm{~d} \sigma e^{-\left(\left|k_{1}\right|^{2}+\left|k_{2}\right|^{2}\right)|s-\sigma|-\left(\left|k+k_{3}\right|^{2}+\left|k_{3}\right|^{2}\right)|t-s|-\left(\left|k_{4}\right|^{2}+\left|k+k_{4}\right|^{2}\right)|t-\sigma|} \\
= & I_{2}^{\varepsilon}(t)(k) .
\end{aligned}
$$

Finally, we have

$$
\sup _{t \in[0, T], \varepsilon} \mathbb{E}\left[\left|\Delta_{q}\left(I\left(X^{\varepsilon}\right)^{\diamond 3} X^{\varepsilon}\right)(t)\right|^{2}\right] \lesssim \rho, T 2^{q(1+\rho)},
$$

which is the wanted bound.

4.5. Renormalization for $I\left(X^{\diamond 2}\right) \circ X^{\diamond 2}$. We only prove the crucial estimate for a renormalization of $I\left(\left(X^{\varepsilon}\right)\right)^{\diamond 2} \circ\left(X^{\varepsilon}\right)^{\diamond 2}$. We recall that since all the other terms of the product $\left(I\left(X^{\varepsilon}\right)^{\diamond 2}\left(X^{\varepsilon}\right)^{\diamond 2}\right)^{\diamond}$ are well defined and converge to a limit with a good regularity.

PROOF OF (4.5). Let us begin by giving the computation for the first term. Indeed a chaos decomposition gives

$$
\begin{aligned}
-\left(I\left(\left(X^{\varepsilon}\right)^{\diamond 2}\right)(t) \circ\left(X_{t}^{\varepsilon}\right)^{\diamond 2}\right) & \\
= & \sum_{k \in \mathbb{Z}^{3}} \sum_{|i-j| \leq 1} \sum_{k_{1234}=k} \theta\left(2^{-i}\left|k_{12}\right|\right) \theta\left(2^{-j}\left|k_{34}\right|\right) \int_{0}^{t} \mathrm{~d} s \\
& \times e^{-\left|k_{12}\right|^{2}|t-s|}: \hat{X}_{s}^{\varepsilon}\left(k_{1}\right) \hat{X}_{s}^{\varepsilon}\left(k_{2}\right) \hat{X}_{t}^{\varepsilon}\left(k_{3}\right) \hat{X}_{t}^{\varepsilon}\left(k_{4}\right): e_{k}
\end{aligned}
$$




$$
\begin{aligned}
& +4 \sum_{\substack{k \in \mathbb{Z}^{3} \\
|i-j| \leq 1 \\
k_{13}=k, k_{2}}} \theta\left(2^{-i}\left|k_{12}\right|\right) \theta\left(2^{-j}\left|k_{2(-3)}\right|\right)\left|f\left(\varepsilon k_{2}\right)\right|^{2} \\
& \times \int_{0}^{t} \mathrm{~d} s \frac{e^{-\left(\left|k_{12}\right|^{2}+\left|k_{2}\right|^{2}\right)|t-s|}}{\left|k_{2}\right|^{2}}: \hat{X}_{s}^{\varepsilon}\left(k_{1}\right) \hat{X}_{t}^{\varepsilon}\left(k_{3}\right): e_{k} \\
& +2 \sum_{\substack{|i-j| \leq 1 \\
k_{1}, k_{2}}} \theta\left(2^{-i}\left|k_{12}\right|\right) \theta\left(2^{-j}\left|k_{12}\right|\right)\left|f\left(\varepsilon k_{1}\right)\right|^{2}\left|f\left(\varepsilon k_{2}\right)\right|^{2} \\
& \times \frac{1-e^{-\left(\left|k_{1}\right|^{2}+\left|k_{2}\right|^{2}+\left|k_{12}\right|^{2}\right) t}}{\left|k_{1}\right|^{2}\left|k_{2}\right|^{2}\left(\left|k_{1}\right|^{2}+\left|k_{2}\right|^{2}+\left|k_{12}\right|^{2}\right)},
\end{aligned}
$$

where : : denotes the usual Gaussian-Wick product (see for example [12]). Let us focus on the last term

$$
\begin{aligned}
A^{\varepsilon}(t)= & \sum_{\substack{|i-j| \leq 1 \\
k_{1}, k_{2}}}\left|\theta\left(2^{-i} k_{12}\right)\right|\left|\theta\left(2^{-j} k_{12}\right)\right|\left|f\left(\varepsilon k_{1}\right)\right|^{2}\left|f\left(\varepsilon k_{2}\right)\right|^{2} \\
& \times \frac{1-e^{-\left(\left|k_{1}\right|^{2}+\left|k_{2}\right|^{2}+\left|k_{12}\right|^{2}\right) t}}{\left|k_{1}\right|^{2}\left|k_{2}\right|^{2}\left(\left|k_{1}\right|^{2}+\left|k_{2}\right|^{2}+\left|k_{12}\right|^{2}\right)} \\
= & C_{2}^{\varepsilon}+I_{3}^{\varepsilon}(t),
\end{aligned}
$$

where $I_{3}^{\varepsilon}$ is defined below. Moreover, it is not difficult to see that

$$
\lim _{\varepsilon \rightarrow 0} C_{2}^{\varepsilon}=\sum_{|i-j| \leq 1} \sum_{k_{1}, k_{2}} \frac{\theta\left(2^{-i}\left|k_{12}\right|\right) \theta\left(2^{-j}\left|k_{12}\right|\right)}{\left|k_{1}\right|^{2}\left|k_{2}\right|^{2}\left(\left|k_{1}\right|^{2}+\left|k_{2}\right|^{2}+\left|k_{12}\right|^{2}\right)}=+\infty
$$

To obtain the needed convergence, we have to estimate the following terms:

$$
\begin{aligned}
I_{1}^{\varepsilon}(t)= & \sum_{\substack{k \in \mathbb{Z}^{3} \\
|i-j| \leq 1 \\
k_{1234}=k}} \theta\left(2^{-i}\left|k_{12}\right|\right) \theta\left(2^{-j}\left|k_{34}\right|\right) \\
& \times \int_{0}^{t} \mathrm{~d} s e^{-\left|k_{12}\right|^{2}|t-s|}: \hat{X}_{s}^{\varepsilon}\left(k_{1}\right) \hat{X}_{s}^{\varepsilon}\left(k_{2}\right) \hat{X}_{t}^{\varepsilon}\left(k_{3}\right) \hat{X}_{t}\left(k_{4}\right): e_{k}, \\
I_{2}^{\varepsilon}(t)= & \sum_{k \in \mathbb{Z}^{3}} \sum_{|i-j| \leq 1} \sum_{k_{13}=k, k_{2}} \theta\left(2^{-i}\left|k_{12}\right|\right) \theta\left(2^{-j}\left|k_{2(-3)}\right|\right)\left|f\left(\varepsilon k_{2}\right)\right|^{2} \\
& \times \int_{0}^{t} \mathrm{~d} s e^{-\left(\left|k_{12}\right|^{2}+\left|k_{2}\right|^{2}\right)|t-s|}\left|k_{2}\right|^{-2} \hat{X}_{s}^{\varepsilon}\left(k_{1}\right) \hat{X}_{t}^{\varepsilon}\left(k_{3}\right) e_{k}
\end{aligned}
$$


and

$$
\begin{aligned}
I_{3}^{\varepsilon}(t)= & \sum_{|i-j| \leq 1} \sum_{k_{1}, k_{2}} \theta\left(2^{-i}\left|k_{12}\right|\right) \theta\left(2^{-j}\left|k_{12}\right|\right) \\
& \times \frac{\left|f\left(\varepsilon k_{1}\right)\right|^{2}\left|f\left(\varepsilon k_{2}\right)\right|^{2} e^{-\left(\left|k_{1}\right|^{2}+\left|k_{2}\right|^{2}+\left|k_{12}\right|^{2}\right) t}}{\left|k_{1}\right|^{2}\left|k_{2}\right|^{2}\left(\left|k_{1}\right|^{2}+\left|k_{2}\right|^{2}+\left|k_{12}\right|^{2}\right)} .
\end{aligned}
$$

We notice that for the deterministic part we have the following bound:

$$
\begin{aligned}
I_{3}^{\varepsilon}(t) & \lesssim \sum_{\substack{k_{1}, k_{2},\left|k_{1}\right| \leq\left|k_{2}\right|}} \frac{t^{-\rho}}{\left|k_{1}\right|^{2}\left|k_{2}\right|^{2}\left(\left|k_{1}\right|^{2}+\left|k_{2}\right|^{2}+\left|k_{12}\right|^{2}\right)^{1+\rho}} \\
& \lesssim t^{-\rho} \sum_{\substack{k_{1}, k_{2},\left|k_{1}\right| \leq\left|k_{2}\right|}}\left|k_{2}\right|^{-4-2 \rho}\left|k_{1}\right|^{-2} \lesssim_{\rho} t^{-\rho}
\end{aligned}
$$

and the dominated convergence gives for $\rho>0$

$$
\sup _{t \in[0, T]} t^{\rho}\left|I_{3}^{\varepsilon}(t)-I_{3}(t)\right| \rightarrow^{\varepsilon \rightarrow 0} 0,
$$

where

$$
I_{3}(t)=\sum_{|i-j| \leq 1} \sum_{k_{1}, k_{2}} \theta\left(2^{-i}\left|k_{12}\right|\right) \theta\left(2^{-j}\left|k_{12}\right|\right) \frac{e^{-\left(\left|k_{1}\right|^{2}+\left|k_{2}\right|^{2}+\left|k_{12}\right|^{2}\right) t}}{\left|k_{1}\right|^{2}\left|k_{2}\right|^{2}\left(\left|k_{1}\right|^{2}+\left|k_{2}\right|^{2}+\left|k_{12}\right|^{2}\right)} .
$$

Let us focus on $I_{1}^{\varepsilon}(t)$ and $I_{2}^{\varepsilon}(t)$. A simple computation gives

$$
\begin{aligned}
\mathbb{E}\left[\Delta_{q}\left|I_{1}^{\varepsilon}(t)\right|^{2}\right] & \\
= & 2 \sum_{k \in \mathbb{Z}^{3}} \sum_{i \sim j \sim i^{\prime} \sim j^{\prime}} \sum_{k_{1234}=k} \theta\left(2^{-i}\left|k_{12}\right|\right) \theta\left(2^{-j}\left|k_{34}\right|\right) \\
& \times \theta\left(2^{-i^{\prime}}\left|k_{12}\right|\right) \theta\left(2^{-j^{\prime}}\left|k_{34}\right|\right) \theta\left(2^{-q}|k|\right)^{2} \\
& \times \prod_{l=1}^{4} \frac{\left|f\left(\varepsilon k_{l}\right)\right|^{2}}{\left|k_{l}\right|^{2}} \int_{0}^{t} \int_{0}^{t} \mathrm{~d} s \mathrm{~d} \sigma e^{-\left|k_{12}\right|^{2}(|t-s|+|t-\sigma|)-\left(\left|k_{1}\right|^{2}+\left|k_{2}\right|^{2}\right)|s-\sigma|} \\
& +2 \sum_{k \in \mathbb{Z}^{3} i \sim j \sim i^{\prime} \sim j^{\prime}} \sum_{k_{1234}=k} \theta\left(2^{-i}\left|k_{12}\right|\right) \theta\left(2^{-j}\left|k_{34}\right|\right) \\
& \times \theta\left(2^{-j^{\prime}}\left|k_{12}\right|\right) \theta\left(2^{-i^{\prime}}\left|k_{34}\right|\right) \theta\left(2^{-q}|k|\right)^{2} \\
& \times \prod_{l=1}^{4} \frac{\left|f\left(\varepsilon k_{l}\right)\right|^{2}}{\left|k_{l}\right|^{2}} \int_{0}^{t} \int_{0}^{t} \mathrm{~d} s \mathrm{~d} \sigma e^{-\left(\left|k_{12}\right|^{2}+\left|k_{1}\right|^{2}+\left|k_{2}\right|^{2}\right)|t-s|-\left(\left|k_{34}\right|^{2}+\left|k_{3}\right|^{2}+\left|k_{4}\right|^{2}\right)|t-\sigma|} \\
& +2 \sum_{k \in \mathbb{Z}^{3} i \sim j ; i^{\prime} \sim j^{\prime}} \sum_{k_{1234}=k} \theta\left(2^{-i}\left|k_{12}\right|\right) \theta\left(2^{-j}\left|k_{34}\right|\right)
\end{aligned}
$$




$$
\begin{aligned}
& \times \theta\left(2^{-i^{\prime}}\left|k_{14}\right|\right) \theta\left(2^{-j^{\prime}}\left|k_{23}\right|\right) \theta\left(2^{-q}|k|\right)^{2} \\
& \times \prod_{l=1}^{4} \frac{\left|f\left(\varepsilon k_{l}\right)\right|^{2}}{\left|k_{l}\right|^{2}} \int_{0}^{t} \int_{0}^{t} \mathrm{~d} s \mathrm{~d} \sigma e^{-\left(\left|k_{12}\right|^{2}+\left|k_{2}\right|^{2}\right)|t-s|-\left(\left|k_{14}\right|^{2}+\left|k_{4}\right|^{2}\right)|t-\sigma|-\left|k_{1}\right|^{2}|s-\sigma|} \\
\equiv & \sum_{j=1}^{3} I_{1, j}^{\varepsilon}(t) .
\end{aligned}
$$

Let us begin by treating the first term. As usual by symmetry, we have

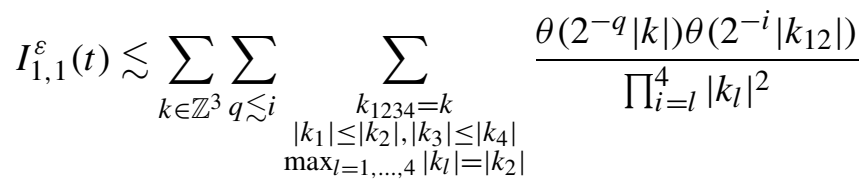

$$
\begin{aligned}
& \times \int_{0}^{t} \int_{0}^{t} \mathrm{~d} s \mathrm{~d} \sigma e^{-\left|k_{12}\right|^{2}(|t-s|+|t-\sigma|)-\left|k_{2}\right|^{2}|s-\sigma|} \\
& +\sum_{k \in \mathbb{Z}^{3}} \sum_{\substack { q \lesssim i \\
\begin{subarray}{c}{k_{1234}=k \\
\left|k_{1}\right| \leq\left|k_{2}\right|,\left|k_{3}\right| \leq\left|k_{4}\right| \\
\max _{l=1, \ldots, 4}\left|k_{l}\right|=\left|k_{4}\right|{ q \lesssim i \\
\begin{subarray} { c } { k _ { 1 2 3 4 } = k \\
| k _ { 1 } | \leq | k _ { 2 } | , | k _ { 3 } | \leq | k _ { 4 } | \\
\operatorname { m a x } _ { l = 1 , \ldots , 4 } | k _ { l } | = | k _ { 4 } | } }\end{subarray}} \frac{\theta\left(2^{-q}|k|\right) \theta\left(2^{-i}\left|k_{12}\right|\right)}{\prod_{i=l}^{4}\left|k_{l}\right|^{2}} \\
& \times \int_{0}^{t} \int_{0}^{t} \mathrm{~d} s \mathrm{~d} \sigma e^{-\left|k_{12}\right|^{2}(|t-s|+|t-\sigma|)-\left|k_{2}\right|^{2}|s-\sigma|} \\
& \equiv A_{1}^{\varepsilon}(t)+A_{2}^{\varepsilon}(t) \text {. }
\end{aligned}
$$

We notice that if $\max _{l=1, \ldots, 4}\left|k_{l}\right|=\left|k_{1}\right|$ then $|k| \lesssim\left|k_{1}\right|$, and

$$
\begin{aligned}
A_{1}^{\varepsilon}(t) & \lesssim \sum_{k \in \mathbb{Z}^{3}}|k|^{-1+2 \eta} \theta\left(2^{-q}|k|\right) \sum_{\substack{k_{1234}=k \\
\left|k_{1}\right| \leq\left|k_{2}\right|,\left|k_{3}\right| \leq\left|k_{4}\right| \\
\max _{l=1, \ldots, 4\left|k_{l}\right|=\left|k_{2}\right|}}}\left(\left|k_{1}\right|\left|k_{3}\right|\left|k_{4}\right|\right)^{-3-\eta / 3} \sum_{q \lesssim i} 2^{-i(2-\eta)} \\
& \lesssim t^{\eta} 2^{3 q \eta},
\end{aligned}
$$

where we have used that

$$
\int_{0}^{t} \int_{0}^{t} \mathrm{~d} s \mathrm{~d} \sigma e^{-\left|k_{12}\right|^{2}(|t-s|+|s-\sigma|)-\left|k_{2}\right|^{2}|s-\sigma|} \lesssim t^{\eta} \frac{1}{\left|k_{2}\right|^{2-\eta}\left|k_{12}\right|^{2-\eta}} .
$$

By a similar argument, we have

$$
\begin{aligned}
A_{2}^{\varepsilon}(t) & \lesssim \sum_{k \in \mathbb{Z}^{3}}|k|^{-1+4 \eta} \theta\left(2^{-q}|k|\right) \sum_{\substack{k_{1234}=k \\
\left|k_{1}\right| \leq\left|k_{2}\right|,\left|k_{3}\right| \leq\left|k_{4}\right| \\
\max _{l=1, \ldots, 4}\left|k_{l}\right|=\left|k_{4}\right|}}\left(\left|k_{1}\right|\left|k_{2}\right|\left|k_{3}\right|\right)^{-3-\eta} \sum_{q \lesssim i} 2^{-i(2-\eta)} \\
& \lesssim t^{\eta} 2^{5 q \eta}
\end{aligned}
$$


and then $\sup _{\varepsilon} I_{1,1}(t) \lesssim t^{\eta} 2^{5 q \eta}$. Let us treat the second term $I_{1,2}^{\varepsilon}(t)$. We have

$$
\begin{aligned}
I_{1,2}^{\varepsilon}(t) \lesssim & \sum_{k \in \mathbb{Z}^{3}} \sum_{q \lesssim i \sim j} \sum_{\substack{k_{1233}=k \\
\left|k_{1}\right| \leq \leq k_{1}|,| k_{k}|\leq| k_{4}\left| \\
\max _{l=1, \ldots, 4}\right| k_{l}|=| k_{2} \mid}} \theta\left(2^{-q}|k|\right) \theta\left(2^{-i}\left|k_{12}\right|\right) \theta\left(2^{-j}\left|k_{34}\right|\right) \prod_{i=l}^{4}\left|k_{l}\right|^{-2} \\
& \times \int_{0}^{t} \int_{0}^{t} \mathrm{~d} s \mathrm{~d} \sigma e^{-\left(\left|k_{12}\right|^{2}+\left|k_{1}\right|^{2}+\left|k_{2}\right|^{2}\right)|t-s|-\left(\left|k_{34}\right|^{2}+\left|k_{3}\right|^{2}+\left|k_{4}\right|^{2}\right)|s-\sigma|} \\
\lesssim & \sum_{k \in \mathbb{Z}^{3}}|k|^{-1+4 \eta} \sum_{\substack{q \leq i \sim j \\
\vdots}} \sum_{\substack{k_{12} 34=k \\
\left|k_{1}\right| \leq\left|k_{2}\right|,\left|k_{3}\right| \leq\left|k_{4}\right| \\
\max _{l=1, \ldots, 4}\left|k_{l}\right|=\left|k_{2}\right|}} \frac{\theta\left(2^{-q}|k|\right) \theta\left(2^{-i}\left|k_{12}\right|\right) \theta\left(2^{-j}\left|k_{34}\right|\right)}{\left|k_{1}\right|^{2}\left|k_{2}\right|^{3+3 \eta}\left|k_{3}\right|^{2}\left|k_{4}\right|^{2}\left|k_{34}\right|^{2-\eta}} \\
\lesssim & t^{\eta} 2^{q(2+4 \eta)} \sum_{q \lesssim j} 2^{-j(2-\eta)} \sum_{l}|l|^{-3-\eta} \lesssim t^{\eta} 2^{5 q \eta} .
\end{aligned}
$$

We have to treat the last term in the fourth chaos. A similar computation gives

$$
\begin{aligned}
I_{1,3}^{\varepsilon}(t) \lesssim & \sum_{k \in \mathbb{Z}^{3}} \sum_{q \lesssim i \sim j ; q \lesssim i^{\prime} \sim j^{\prime}} \sum_{k_{1234}=k} \theta\left(2^{-i}\left|k_{12}\right|\right) \theta\left(2^{-j}\left|k_{34}\right|\right) \\
& \times \theta\left(2^{-i^{\prime}}\left|k_{14}\right|\right) \theta\left(2^{-j^{\prime}}\left|k_{23}\right|\right) \theta\left(2^{-q}|k|\right)^{2} \\
& \times \int_{0}^{t} \int_{0}^{t} \mathrm{~d} s \mathrm{~d} \sigma e^{-\left(\left|k_{12}\right|^{2}+\left|k_{2}\right|^{2}\right)|t-s|-\left(\left|k_{14}\right|^{2}+\left|k_{4}\right|^{2}\right)|t-\sigma|} \\
\lesssim & \sum_{k \in \mathbb{Z}^{3}} \sum_{q \lesssim i^{\prime} \sim j^{\prime}} \sum_{\left|k_{4}\right| \leq\left|k_{2}\right|,\left|k_{1}\right| \leq\left|k_{3}\right|} \theta\left(2^{-i}\left|k_{12}\right|\right) \\
& \times \theta\left(2^{-j}\left|k_{34}\right|\right) \theta\left(2^{-i^{\prime}}\left|k_{14}\right|\right) \theta\left(2^{-q}|k|\right)^{2} \prod_{l=1}^{4} \frac{1}{\left|k_{l}\right|^{2}} \\
& \times \int_{0}^{t} \int_{0}^{t} \mathrm{~d} s \mathrm{~d} \sigma e^{-\left|k_{2}\right|^{2}|t-s|-\left|k_{14}\right|^{2}|t-\sigma|} \\
\lesssim & t^{\eta} 2^{-q(2-\eta)} \sum_{k \in \mathbb{Z}^{3}} \theta\left(2^{-q}|k|\right) \sum_{\substack{k_{1234}=k \\
\left|k_{4}\right| \leq\left|k_{2}\right|,\left|k_{1}\right| \leq\left|k_{3}\right|}} \frac{1}{\left|k_{1}\right|^{2}\left|k_{2}\right|^{4-\eta}\left|k_{3}\right|^{2}\left|k_{4}\right|^{2}} .
\end{aligned}
$$

We still need to bound the sum

$$
\sum_{\substack{k_{1234}=k \\\left|k_{4}\right| \leq\left|k_{2}\right|,\left|k_{1}\right| \leq\left|k_{3}\right|}} \frac{1}{\left|k_{1}\right|^{2}\left|k_{2}\right|^{4-\eta}\left|k_{3}\right|^{2}\left|k_{4}\right|^{2}} .
$$

For that, we notice that when $\left|k_{3}\right| \leq\left|k_{2}\right|$ we can use the bound

$$
\frac{1}{\left|k_{1}\right|^{2}\left|k_{2}\right|^{4-\eta}\left|k_{3}\right|^{2}\left|k_{4}\right|^{2}} \lesssim|k|^{-1+4 \eta}\left|k_{1}\right|^{-3-\eta}\left|k_{3}\right|^{-3-\eta}\left|k_{4}\right|^{-3-\eta}
$$


and when $\left|k_{2}\right| \leq\left|k_{3}\right|$ we can use that

$$
\begin{aligned}
\frac{1}{\left|k_{1}\right|^{2}\left|k_{2}\right|^{4-\eta}\left|k_{3}\right|^{2}\left|k_{4}\right|^{2}} & \lesssim|k|^{-1+4 \eta}\left|k_{1}\right|^{-2}\left|k_{2}\right|^{-4+\eta}\left|k_{3}\right|^{-1+4 \eta}\left|k_{4}\right|^{-2} \\
& \lesssim|k|^{-1+4 \eta}\left(\left|k_{1}\right|\left|k_{2}\right|\left|k_{4}\right|\right)^{-3-\eta}
\end{aligned}
$$

where we have used that $\left|k_{4}\right| \leq\left|k_{2}\right|$. We can conclude that $\sup _{\varepsilon} I_{1,3}^{\varepsilon}(t) \lesssim t^{\eta} 2^{5 q \eta}$. This gives the needed bound for the term lying in the chaos of order four; in fact, we have

$$
\sup _{\varepsilon} \mathbb{E}\left[\Delta_{q}\left|I_{1}^{\varepsilon}(t)\right|^{2}\right] \lesssim t^{\eta} 2^{5 q \eta}
$$

Let us focus on the term lying in the second chaos:

$$
\begin{aligned}
\mathbb{E}\left[\left|\Delta_{q} I_{2}^{\varepsilon}(t)\right|^{2}\right] & \sum_{k \in \mathbb{Z}^{3}} \sum_{q \lesssim i \sim j, q \lesssim i^{\prime} \sim j^{\prime}} \sum_{k_{13}=k, k_{2}, k_{4}} \theta\left(2^{-i}\left|k_{12}\right|\right) \theta\left(2^{j}\left|k_{2(-3)}\right|\right) \\
& \times \theta\left(2^{-i^{\prime}}\left|k_{14}\right|\right) \theta\left(2^{-j^{\prime}}\left|k_{4(-3)}\right|\right) \\
& \times \prod_{i=1}^{4} \frac{\left|f\left(\varepsilon k_{l}\right)\right|^{2}}{\left|k_{l}\right|^{2}} \int_{0}^{t} \int_{0}^{t} \mathrm{~d} s \mathrm{~d} \sigma e^{-\left(\left|k_{12}\right|^{2}+\left|k_{2}\right|^{2}\right)|t-s|-\left(\left|k_{14}\right|^{2}+\left|k_{4}\right|^{2}\right)|t-\sigma|-\left|k_{1}\right|^{2}|s-\sigma|} \\
& +\sum_{k \in \mathbb{Z}^{3}} \sum_{q \lesssim i \sim j, q \lesssim i^{\prime} \sim j^{\prime}} \sum_{k_{13}=k, k_{2}, k_{4}} \theta\left(2^{-i}\left|k_{12}\right|\right) \theta\left(2^{j}\left|k_{2(-3)}\right|\right) \\
& \times \theta\left(2^{-i^{\prime}}\left|k_{34}\right|\right) \theta\left(2^{-j^{\prime}}\left|k_{4(-3)}\right|\right) \\
& \times \prod_{i=1}^{4} \frac{\left|f\left(\varepsilon k_{l}\right)\right|^{2}}{\left|k_{l}\right|^{2}} \int_{0}^{t} \int_{0}^{t} \mathrm{~d} s \mathrm{~d} \sigma e^{-\left(\left|k_{12}\right|^{2}+\left|k_{2}\right|^{2}+\left|k_{1}\right|^{2}\right)|t-s|-\left(\left|k_{34}\right|^{2}+\left|k_{4}\right|^{2}+\left|k_{3}\right|^{2}\right)|t-\sigma|} \\
\equiv & I_{2,1}^{\varepsilon}(t)+I_{2,2}^{\varepsilon}(t) .
\end{aligned}
$$

We treat these two terms separately. In fact, by symmetry, we have

$$
\begin{aligned}
I_{2,1}^{\varepsilon}(t) \lesssim & \sum_{k \in \mathbb{Z}^{3}} \sum_{q \lesssim i \sim j ; q \lesssim i^{\prime \prime} j^{\prime}} \sum_{\substack{k_{13}=k \\
k_{2}, k_{4},\left|k_{1}\right| \leq\left|k_{3}\right|}} \theta\left(2^{-q}|k|\right)^{2} \theta\left(2^{-i}\left|k_{12}\right|\right) \\
& \times \theta\left(2^{j}\left|k_{2(-3)}\right|\right) \theta\left(2^{-i^{\prime}}\left|k_{14}\right|\right) \theta\left(2^{-j^{\prime}}\left|k_{4(-3)}\right|\right) \\
& \times \prod_{i=1}^{4} \frac{\left|f\left(\varepsilon k_{l}\right)\right|^{2}}{\left|k_{l}\right|^{2}} \int_{0}^{t} \int_{0}^{t} \mathrm{~d} s \mathrm{~d} \sigma e^{-\left(\left|k_{12}\right|^{2}+\left|k_{2}\right|^{2}\right)|t-s|-\left(\left|k_{14}\right|^{2}+\left|k_{4}\right|^{2}\right)|t-\sigma|} \\
\lesssim & t^{\eta} \sum_{|k| \sim q}|k|^{-1+\eta} \sum_{q \lesssim i, i^{\prime}} \theta\left(2^{-i}\left|k_{12}\right|\right) \theta\left(2^{-i^{\prime}}\left|k_{14}\right|\right)
\end{aligned}
$$




$$
\begin{aligned}
& \times \sum_{k_{1}, k_{2}, k_{4}}\left|k_{1}\right|^{-3-\eta}\left|k_{2}\right|^{-3-\eta}\left|k_{3}\right|^{-3-\eta}\left|k_{12}\right|^{-1+2 \eta}\left|k_{14}\right|^{-1+2 \eta} \\
\lesssim & 2^{q(2+\eta)} \sum_{q \lesssim i, i^{\prime}} 2^{-\left(i+i^{\prime}\right)(1-2 \eta)} \lesssim t^{\eta} 2^{3 q \eta}
\end{aligned}
$$

which gives the first bound. The second term has a similar bound. Indeed

$$
\begin{aligned}
I_{2,2}^{\varepsilon}(t) \lesssim & \sum_{k \in \mathbb{Z}^{3}} \sum_{q \lesssim i, q \lesssim i^{\prime}} \sum_{k_{13}=k, k_{2}, k_{4},\left|k_{1}\right| \leq\left|k_{3}\right|} \theta\left(2^{-i}\left|k_{12}\right|\right) \theta\left(2^{-i^{\prime}}\left|k_{34}\right|\right) \prod_{i=1}^{4} \frac{\left|f\left(\varepsilon k_{l}\right)\right|^{2}}{\left|k_{l}\right|^{2}} \\
& \times \int_{0}^{t} \int_{0}^{t} \mathrm{~d} s \mathrm{~d} \sigma e^{-\left(\left|k_{12}\right|^{2}+\left|k_{2}\right|^{2}\right)|t-s|-\left(\left|k_{34}\right|^{2}+\left|k_{4}\right|^{2}\right)|t-\sigma|} \lesssim t^{\eta} 2^{3 q \eta},
\end{aligned}
$$

which completes the proof.

4.6. Renormalization for $I\left(X^{\diamond 3}\right) \circ X^{\diamond 2}$. Here again, we only give the crucial bound, but for $I\left(X^{\diamond 3}\right) \diamond X^{\diamond 2}$ instead of $\pi_{0 \diamond}\left(I\left(X^{\diamond 3}\right), X^{\diamond 2}\right)$.

Proposition 4.7. For all $T>0, t \in[0, T], \delta, \delta^{\prime}>0$ and all $1 \gg v>0$ small enough, there exists two constants and $C>0$ depending on $T, \delta, \delta^{\prime}$ and $v$ such that for all $q \geq-1$,

$$
\mathbb{E}\left[t^{\delta^{\prime}+\delta}\left|\Delta_{q}\left(I\left(\left(X_{t}^{\varepsilon}\right)^{\diamond 3}\right)\left(X_{t}^{\varepsilon}\right)^{\diamond 2}-3 C_{2}^{\varepsilon} X_{t}^{\varepsilon}\right)\right|^{2}\right] \leq C t^{\delta} 2^{q(1+v)} .
$$

PROOF. Thanks to a straightforward computation we have

$$
-I\left(\left(X_{t}^{\varepsilon}\right)^{\diamond 3}\right)\left(X_{t}^{\varepsilon}\right)^{\diamond 2}=I_{t}^{(1)}+I_{t}^{(2)}+I_{t}^{(3)},
$$

where

$$
\begin{aligned}
I_{t}^{(1)}= & \sum_{\substack{k \neq 0\\
}} e_{k} \\
& \times \sum_{\substack{k_{12345}=k \\
k_{i} \neq 0}} \int_{0}^{t} \mathrm{~d} s e^{-\left|k_{1}+k_{2}+k_{3}\right|^{2}|t-s|}: \hat{X}_{s}^{\varepsilon}\left(k_{1}\right) \hat{X}_{s}^{\varepsilon}\left(k_{2}\right) \hat{X}_{s}^{\varepsilon}\left(k_{3}\right) \hat{X}_{t}^{\varepsilon}\left(k_{4}\right) \hat{X}_{t}^{\varepsilon}\left(k_{5}\right): \\
I_{t}^{(2)}= & 6 \sum_{\substack{k \neq 0 \\
k_{3}, k_{124}=k \\
k_{i} \neq 0}} e_{k} \\
& \times \int_{0}^{t} \mathrm{~d} s e^{-\left|k_{1}+k_{2}+k_{3}\right|^{2}|t-s|} \frac{e^{-\left|k_{3}\right|^{2}|t-s|}}{\left|k_{3}\right|^{2}} f\left(\varepsilon k_{3}\right)^{2}: \hat{X}_{s}^{\varepsilon}\left(k_{1}\right) \hat{X}_{s}^{\varepsilon}\left(k_{2}\right) \hat{X}_{t}^{\varepsilon}\left(k_{4}\right):
\end{aligned}
$$

and

$I_{t}^{(3)}=6 \sum_{k \neq 0} e_{k} \int_{0}^{t} \mathrm{~d} s \sum_{k_{1} \neq 0, k_{2} \neq 0} \frac{f\left(\varepsilon k_{1}\right)^{2} f\left(\varepsilon k_{2}\right)^{2}}{\left|k_{1}\right|^{2}\left|k_{2}\right|^{2}} e^{-\left(\left|k+k_{1}+k_{2}\right|^{2}+\left|k_{1}\right|^{2}+\left|k_{2}\right|^{2}\right)|t-s|} \hat{X}_{s}^{\varepsilon}(k)$. 
Hence,

$$
\begin{aligned}
& -\left(I\left(\left(X_{t}^{\varepsilon}\right)^{\diamond 3}\right)\left(X_{t}^{\varepsilon}\right)^{\diamond 2}-3 C_{2}^{\varepsilon} X_{t}^{\varepsilon}\right) \\
& =I\left(\left(X_{t}^{\varepsilon}\right)^{\diamond 3}\right)\left(X_{t}^{\varepsilon}\right)^{\diamond 2} I_{t}^{(3)}+\left(I_{t}^{(3)}-\tilde{I}_{t}^{(3)}\right) \\
& \quad+\left(\tilde{I}_{t}^{(3)}-3 \tilde{C}_{2}^{\varepsilon}(t) X_{t}^{\varepsilon}\right)+3\left(C_{2}^{\varepsilon}-\tilde{C}_{2}^{\varepsilon}(t)\right) X_{t}^{\varepsilon},
\end{aligned}
$$

where we remind that

$$
C_{2}^{\varepsilon}=\sum_{k_{1} \neq 0, k_{2} \neq 0} \frac{f\left(\varepsilon k_{1}\right) f\left(\varepsilon k_{2}\right)}{\left|k_{1}\right|^{2}\left|k_{2}\right|^{2}\left(\left|k_{1}\right|^{2}+\left|k_{2}\right|^{2}+\left|k_{1}+k_{2}\right|^{2}\right)}
$$

and where we have defined

$$
\tilde{I}_{t}^{(3)}=6 \sum_{k \neq 0} e_{k} \int_{0}^{t} \mathrm{~d} s \sum_{k_{1} \neq 0, k_{2} \neq 0} \frac{f\left(\varepsilon k_{1}\right)^{2} f\left(\varepsilon k_{2}\right)^{2}}{\left|k_{1}\right|^{2}\left|k_{2}\right|^{2}} e^{-\left(\left|k+k_{1}+k_{2}\right|^{2}+\left|k_{1}\right|^{2}+\left|k_{2}\right|^{2}\right)|t-s|} \hat{X}_{t}^{\varepsilon}(k)
$$

and

$$
\tilde{C}_{2}^{\varepsilon}=2 \int_{0}^{t} \mathrm{~d} s \sum_{k_{1} \neq 0, k_{2} \neq 0} \frac{f\left(\varepsilon k_{1}\right)^{2} f\left(\varepsilon k_{2}\right)^{2}}{\left|k_{1}\right|^{2}\left|k_{2}\right|^{2}} e^{-\left(\left|k_{1}+k_{2}\right|^{2}+\left|k_{1}\right|^{2}+\left|k_{2}\right|^{2}\right)|t-s|} .
$$

Hence for $q \geq-1$,

$$
\begin{aligned}
& \mathbb{E}\left[\mid \Delta_{q}\right.\left.\left.\left(I\left(\left(X_{t}^{\varepsilon}\right)^{\diamond 3}\right)\left(X_{t}^{\varepsilon}\right)^{\diamond 2}-3 C_{2}^{\varepsilon} X_{t}^{\varepsilon}\right)\right|^{2}\right] \\
& \lesssim \mathbb{E}\left[\left|\Delta_{q}\left(I_{t}^{(1)}\right)\right|^{2}\right]+\mathbb{E}\left[\left|\Delta_{q}\left(I_{t}^{(2)}\right)\right|^{2}\right]+\mathbb{E}\left[\left|\Delta_{q}\left(I_{t}^{(3)}-\tilde{I}_{t}^{(3)}\right)\right|^{2}\right] \\
& \quad+\mathbb{E}\left[\left|\Delta_{q}\left(\tilde{I}_{t}^{(3)}-\tilde{C}_{2}^{\varepsilon}(t) X_{t}^{\varepsilon}\right)\right|^{2}\right]+\left|C_{2}^{\varepsilon}-\tilde{C}_{2}^{\varepsilon}(t)\right|^{2} \mathbb{E}\left[\left|\Delta_{q} X_{t}^{\varepsilon}\right|^{2}\right]
\end{aligned}
$$

Terms in the first chaos. Let us first deal with the "deterministic" part. Here, $C_{2}^{\varepsilon}-\tilde{C}_{2}^{\varepsilon}(t)$. An obvious computation gives for all $\delta^{\prime}>0,\left|C_{2}^{\varepsilon}-\tilde{C}_{2}^{\varepsilon}(t)\right|^{2} \lesssim \delta^{\prime} 1 / t^{\delta^{\prime}}$. Furthermore, $\mathbb{E}\left[\left|\Delta_{q} X_{t}^{\varepsilon}\right| 2\right] \lesssim 2^{q}$, hence for all $\delta^{\prime}>0$,

$$
\left|C_{2}^{\varepsilon}-\tilde{C}_{2}^{\varepsilon}(t)\right|^{2} \mathbb{E}\left[\left|\Delta_{q} X_{t}^{\varepsilon}\right|^{2}\right] \lesssim 2^{q} / t^{\delta^{\prime}}
$$

Let us deal with $\mathbb{E}\left[\left|\Delta_{q}\left(I_{t}^{(3)}-\tilde{I}_{t}^{(3)}\right)\right|^{2}\right]$. For $k \neq 0$, we define

$$
a_{k}(t-s)=\sum_{k_{1} \neq 0, k_{2} \neq 0} \frac{f\left(\varepsilon k_{1}\right)^{2} f\left(\varepsilon k_{2}\right)^{2}}{\left|k_{1}\right|^{2}\left|k_{2}\right|^{2}} e^{-\left(\left|k+k_{1}+k_{2}\right|^{2}+\left|k_{1}\right|^{2}+\left|k_{2}\right|^{2}\right)|t-s|},
$$

such that

$$
\begin{aligned}
& \mathbb{E}\left[\left|\Delta_{q}\left(I_{t}^{(3)}-\tilde{I}_{t}^{(3)}\right)\right|^{2}\right] \\
& \quad=\mathbb{E}\left[\left|\int_{0}^{t} \sum_{k} \theta\left(2^{-q} k\right) e_{k} a_{k}(t-s)\left(\hat{X}_{s}^{\varepsilon}(k)-\hat{X}_{t}^{\varepsilon}(k)\right)\right|^{2}\right]
\end{aligned}
$$




$$
\begin{aligned}
= & \int_{[0, t]^{2}} \mathrm{~d} \bar{s} \mathrm{~d} s \sum_{\substack{k \neq 0 \\
\bar{k} \neq 0}} e_{k} e_{\bar{k}} \theta\left(2^{-q} k\right) \theta\left(2^{-q} \bar{k}\right) \\
& \times a_{k}(t-s) a_{\bar{k}}(t-\bar{s}) \mathbb{E}\left[\left(\hat{X}_{s}^{\varepsilon}(k)-\hat{X}_{t}^{\varepsilon}(k)\right)\left(\hat{X}_{\bar{s}}^{\varepsilon}(\bar{k})-\hat{X}_{t}^{\varepsilon}(\bar{k})\right)\right] .
\end{aligned}
$$

But

$$
\begin{aligned}
& \mathbb{E}\left[\left(\hat{X}_{s}^{\varepsilon}(k)-\hat{X}_{t}^{\varepsilon}(k)\right)\left(\hat{X}_{\bar{s}}^{\varepsilon}(\bar{k})-\hat{X}_{t}^{\varepsilon}(\bar{k})\right)\right] \\
& \quad=\delta_{k=-\bar{k}} \frac{f(\varepsilon k)^{2}}{|k|^{2}}\left(e^{-|s-\bar{s}||k|^{2}}-e^{-|t-\bar{s}||k|^{2}}-e^{-|t-s||k|^{2}}+1\right) \\
& \quad \lesssim \delta_{k=-\bar{k}} \frac{f(\varepsilon k)^{2}}{|k|^{2}}|k|^{2 \eta}|t-s|^{\eta / 2}|t-\bar{s}|^{\eta / 2} .
\end{aligned}
$$

Hence

$$
\mathbb{E}\left[\left|\Delta_{q}\left(I_{t}^{(3)}-\tilde{I}_{t}^{(3)}\right)\right|^{2}\right] \lesssim \sum_{k \neq 0} \theta\left(2^{-q} k\right)^{2} \frac{f(\varepsilon k)^{2}}{|k|^{2(1-\eta)}}\left(\int_{0}^{t} \mathrm{~d} s|t-s|^{\eta / 2} a_{k}(|t-s|)\right)^{2}
$$

and

$$
\begin{aligned}
\int_{0}^{t} \mathrm{~d} s|t-s|^{\eta / 2} a_{k}(|t-s|) & \\
& =\sum_{\substack{k_{1} \neq 0 \\
k_{2} \neq 0}} \int_{0}^{t} \mathrm{~d} s|t-s|^{\eta / 2} e^{-\left(\left|k+k_{1}+k_{2}\right|^{2}+\left|k_{1}\right|^{2}+\left|k_{2}\right|^{2}\right)|t-s|} \frac{f\left(\varepsilon k_{1}\right)^{2} f\left(\varepsilon k_{1}\right)^{2}}{\left|k_{1}\right|^{2}\left|k_{2}\right|^{2}} \\
& \lesssim \sum_{\substack{k_{1} \neq 0 \\
k_{2} \neq 0}}\left|k_{1}\right|^{-3-\eta^{\prime}}\left|k_{2}\right|^{-3-\eta^{\prime}} \int_{0}^{t} \mathrm{~d} s|t-s|^{-1+\left(\eta / 2-\eta^{\prime}\right)} \lesssim t^{\eta / 2-\eta^{\prime}},
\end{aligned}
$$

for $\eta / 2-\eta^{\prime}>0$. Hence we have

$$
\mathbb{E}\left[\left|\Delta_{q}\left(I_{t}^{(3)}-\tilde{I}_{t}^{(3)}\right)\right|^{2}\right] \lesssim 2^{q(1+2 \eta)} t^{\eta-2 \eta^{\prime}} .
$$

We have furthermore

$$
\mathbb{E}\left[\left|\Delta_{q}\left(\tilde{I}_{t}^{(3)}-C_{2}^{\varepsilon} X_{t}^{\varepsilon}\right)\right|^{2}\right]=\sum_{k \neq 0} \frac{f(\varepsilon k)^{2}}{|k|^{2}} \theta\left(2^{-q} k\right)^{2} b_{k}(t)^{2},
$$

with

$$
\begin{aligned}
b_{k}(t)= & \int_{0}^{t} \sum_{\substack{k_{1} \neq 0 \\
k_{2} \neq 0}} \frac{f\left(\varepsilon k_{1}\right)^{2} f\left(\varepsilon k_{2}\right)^{2}}{\left|k_{1}\right|^{2}\left|k_{2}\right|^{2}} e^{-\left(\left|k_{1}\right|^{2}+\left|k_{2}\right|^{2}\right)|t-s|} \\
& \times\left\{e^{-\left|k_{1}+k_{2}\right|^{2}|t-s|}-e^{-\left|k_{1}+k_{2}+k\right|^{2}|t-s|}\right\} .
\end{aligned}
$$


Using that

$$
\left|e^{-\left|k_{1}+k_{2}+k\right|^{2}|t-s|}-e^{-\left|k_{1}+k_{2}\right|^{2}|t-s|}\right| \lesssim|t-s|^{\eta}|k|^{\eta}\left(|k|+\max \left\{\left|k_{1}\right|,\left|k_{2}\right|\right\}\right)^{\eta}
$$

we have the following bound:

$$
\begin{aligned}
b_{k}(t) \lesssim & \int_{0}^{t} \sum_{\substack{k_{1} \neq 0 \\
k_{2} \neq 0}}\left|k_{1}\right|^{-3-\eta^{\prime}}\left|k_{2}\right|^{-3-\eta^{\prime \prime}}|k|^{\eta} \\
& \times\left(|k|+\max \left\{\left|k_{1}\right|,\left|k_{2}\right|\right\}\right)^{\eta}|t-s|^{-1+\left(\eta-\eta^{\prime} / 2-\eta^{\prime \prime} / 2\right)} .
\end{aligned}
$$

We can suppose that $\max \left\{\left|k_{1}\right|,\left|k_{2}\right|\right\}=\left|k_{1}\right|$ as the expression is symmetric in $k_{1}, k_{2}$, then if $|k|>\left|k_{1}\right|$,

$$
b_{k}(t) \lesssim t^{\left(\eta-\eta^{\prime} / 2-\eta^{\prime \prime} / 2\right)}|k|^{2 \eta}
$$

for $\eta-\eta^{\prime} / 2-\eta^{\prime \prime} / 2>0$. Furthermore, if $\left|k_{1}\right|>|k|$, and $\eta^{\prime}>\eta$ then

$$
b_{k}(t) \lesssim t^{\left(\eta-\eta^{\prime} / 2-\eta^{\prime \prime} / 2\right)}|k|^{\eta} \sum_{\substack{k_{1} \neq 0 \\ k_{2} \neq 0}}\left|k_{1}\right|^{-3-\left(\eta^{\prime}-\eta\right)}\left|k_{2}\right|^{-3-\eta^{\prime \prime}} \lesssim t^{\left(\eta-\eta^{\prime} / 2-\eta^{\prime \prime} / 2\right)}|k|^{\eta} .
$$

Hence there exists $\delta>0$ and $v>0$ such that

$$
\mathbb{E}\left[\left|\Delta_{q}\left(\tilde{I}_{t}^{(3)}-3 C_{2}^{\varepsilon} X_{t}^{\varepsilon}\right)\right|^{2}\right] \lesssim t^{\delta} 2^{(1+v) q} .
$$

Terms in the third chaos. Let us define $c_{k_{1}, k_{2}}(t-s)=\sum_{k_{3} \neq 0} \frac{f\left(\varepsilon k_{3}\right)^{2}}{\left|k_{3}\right|^{2}} \times$ $e^{-\left(\left|k_{1}+k_{2}+k_{3}\right|^{2}+\left|k_{3}\right|^{2}\right)|t-s|}$ such that

$$
I_{t}^{(2)}=6 \sum_{\substack{k \neq 0, k_{i} \neq 0 \\ k_{124}=k}} e_{k} \int_{0}^{t} \mathrm{~d} s c_{k_{1}, k_{2}}(t-s): \hat{X}_{s}\left(k_{1}\right) \hat{X}_{s}\left(k_{2}\right) \hat{X}_{t}^{\varepsilon}\left(k_{4}\right): .
$$

But for all suitable variables we have

$$
\begin{aligned}
& \mathbb{E}\left[: \hat{X}_{s}^{\varepsilon}\left(k_{1}\right) \hat{X}_{s}^{\varepsilon}\left(k_{2}\right) \hat{X}_{t}^{\varepsilon}\left(k_{4}\right):: \hat{X}_{\bar{s}}^{\varepsilon}\left(\bar{k}_{1}\right) \hat{X}_{\bar{s}}^{\varepsilon}\left(\bar{k}_{2}\right) \hat{X}_{t}^{\varepsilon}\left(\bar{k}_{4}\right):\right] \\
&= 2 \delta_{k_{1}=-\bar{k}_{1}} \frac{f\left(\varepsilon k_{1}\right)^{2}}{\left|k_{1}\right|^{2}} \delta_{k_{2}=-\bar{k}_{3}} \frac{f\left(\varepsilon k_{2}\right)^{2}}{\left|k_{2}\right|^{2}} \delta_{k_{3}=-\bar{k}_{3}} \frac{f\left(\varepsilon k_{3}\right)^{2}}{\left|k_{3}\right|^{2}} e^{-\left(\left|k_{1}\right|^{2}+\left|k_{2}\right|^{2}\right)|s-\bar{s}|} \\
&+2 \delta_{k_{1}=-\bar{k}_{1}} \frac{f\left(\varepsilon k_{1}\right)^{2}}{\left|k_{1}\right|^{2}} \delta_{k_{2}=-\bar{k}_{3}} \frac{f\left(\varepsilon k_{2}\right)^{2}}{\left|k_{2}\right|^{2}} \delta_{k_{3}=-\bar{k}_{2}} \frac{f\left(\varepsilon k_{3}\right)^{2}}{\left|k_{3}\right|^{2}} e^{-\left|k_{1}\right|^{2}|s-\bar{s}|} \\
& \times e^{-\left(\left|k_{3}\right|^{2}\right)|t-\bar{s}|} e^{-\left(\left|k_{2}\right|^{2}\right)|t-s|} e^{-\left|k_{1}\right|^{2}|s-\bar{s}|} e^{-\left(\left|k_{3}\right|^{2}\right)|t-\bar{s}|} .
\end{aligned}
$$


By another easy computation, the following holds $\mathbb{E}\left[\left|\Delta_{q}\left(I_{t}^{(2)}\right)\right|^{2}\right]=E_{t}^{2,1}+E_{t}^{2,2}$ where

$$
\begin{aligned}
E_{t}^{2,1}= & 2 \int_{0}^{t} \mathrm{~d} s \int_{0}^{s} \mathrm{~d} \bar{s} \sum_{\substack{k, k_{i} \neq 0 \\
k_{124}=k}} \theta\left(2^{-q} k\right)^{2} \prod_{i} \frac{f\left(\varepsilon k_{i}\right)^{2}}{\left|k_{i}\right|^{2}} c_{k_{1}, k_{2}}(t-s) \\
& \times c_{k_{1}, k_{2}}(t-\bar{s}) e^{-\left(\left|k_{1}\right|^{2}+\left|k_{2}\right|^{2}\right)|s-\bar{s}|}
\end{aligned}
$$

and

$$
\begin{aligned}
E_{t}^{2,1}= & 2 \int_{0}^{t} \mathrm{~d} s \int_{0}^{s} \mathrm{~d} \bar{s} \sum_{\substack{k \neq 0, k_{i} \neq 0, k_{124}=k}} \theta\left(2^{-q} k\right)^{2} \prod_{i} \frac{f\left(\varepsilon k_{i}\right)^{2}}{\left|k_{i}\right|^{2}} \\
& \times c_{k_{1}, k_{2}}(t-s) c_{k_{1}, k_{4}}(t-\bar{s}) e^{-\left|k_{1}\right|^{2}|s-\bar{s}|} e^{-\left|k_{4}\right|^{2}|t-\bar{s}|} e^{-\left|k_{2}\right|^{2}|t-s|} .
\end{aligned}
$$

In $E_{t}^{2,1}$, we have a symmetry in $k_{1}, k_{2}$, hence we can assume that $\left|k_{1}\right| \geq\left|k_{2}\right|$. Furthermore, we have $c_{k_{1}, k_{2}}(t-s) \lesssim|t-s|^{-\frac{1+\eta}{2}}$ and $c_{k_{1}, k_{2}}(t-\bar{s}) \lesssim|s-\bar{s}|^{-\frac{1+\eta}{2}}$. If we assume that $\left|k_{1}\right| \geq\left|k_{4}\right|$ and that $\eta^{\prime} / 2-\eta>0$, we have

$$
\begin{aligned}
& E_{t}^{2,1} \lesssim \int_{0}^{t} \mathrm{~d} s \int_{0}^{s} \mathrm{~d} \bar{s}|t-s|^{-\frac{1+\eta}{2}}|s-\bar{s}|^{-1+\left(\eta^{\prime} / 2-\eta\right)} \\
& \times \sum_{\substack{k \neq 0, k_{i} \neq 0, k_{124}=k}} \theta\left(2^{-q} k\right)^{2} \frac{1}{\left|k_{1}\right|^{3-\eta^{\prime}}\left|k_{2}\right|^{2}\left|k_{4}\right|^{2}} \\
& \lesssim t^{\delta} \sum_{k \neq 0} \frac{\theta\left(2^{-q} k\right)^{2}}{|k|^{1-\eta^{\prime \prime}}} \sum_{k_{2}, k_{3}}\left|k_{2}\right|^{-3-\frac{\eta^{\prime \prime}-\eta^{\prime}}{2}}\left|k_{4}\right|^{-3-\frac{\eta^{\prime \prime}-\eta^{\prime}}{2}} \\
& \lesssim t^{\delta} 2^{q\left(2+\eta^{\prime \prime}\right)},
\end{aligned}
$$

for $\eta^{\prime \prime}>\eta^{\prime}$. When $\left|k_{4}\right| \geq\left|k_{1}\right|$, it is pretty much the same computation.

In $E_{t}^{2,2}$, we can assume that $\left|k_{2}\right| \geq\left|k_{4}\right|$, hence

$$
\begin{aligned}
E_{t}^{2,2} \lesssim & \int_{[0, t]^{2}} \mathrm{~d} s \mathrm{~d} \bar{s} \sum_{\substack{k \neq 0, k_{i} \neq 0, k_{124}=k \\
\left|k_{2}\right| \lesssim\left|k_{4}\right|}} \theta\left(2^{-q} k\right)^{2}\left(\left|k_{1}\right|\left|k_{2}\right|\right)^{-3+\eta^{\prime}} \\
& \times\left|k_{4}\right|^{2}|t-s|^{-1+\frac{\eta^{\prime}-\eta}{2}}|s-\bar{s}|^{-1+\frac{\eta^{\prime}-\eta}{2}} \\
\lesssim & t^{\delta} \sum_{\substack{k \neq 0, k_{i} \neq 0, k_{124}=k}} \theta\left(2^{-q} k\right)^{2}|k|^{-1+\eta^{\prime \prime}}\left|k_{1}\right|^{-3+\eta^{\prime}}\left|k_{2}\right|^{-3+\eta^{\prime}}\left|k_{4}\right|^{2} \max \left(\left|k_{i}\right|\right)^{1-\eta^{\prime \prime}} \\
\lesssim & t^{\delta} 2^{q\left(1+\eta^{\prime \prime}\right)} .
\end{aligned}
$$

Finally, by decomposing the previous expression depending on $\left|k_{1}\right| \geq\left|k_{4}\right|$ or $\left|k_{4}\right| \geq$ $\left|k_{1}\right|$ we have the wanted bound. 
Terms in the fifth chaos. For all suitable variables, we have

$$
\begin{aligned}
\mathbb{E}\left[: \hat{X}_{s}^{\varepsilon}\left(k_{1}\right) \hat{X}_{s}^{\varepsilon}\left(k_{2}\right) \hat{X}_{s}^{\varepsilon}\left(k_{3}\right) \hat{X}_{t}^{\varepsilon}\left(k_{4}\right) \hat{X}_{t}^{\varepsilon}\left(k_{5}\right):: \hat{X}_{\bar{s}}^{\varepsilon}\left(\bar{k}_{1}\right) \hat{X}_{\bar{s}}^{\varepsilon}\left(\bar{k}_{2}\right) \hat{X}_{\bar{s}}^{\varepsilon}\left(\bar{k}_{3}\right) \hat{X}_{t}^{\varepsilon}\left(\bar{k}_{4}\right) \hat{X}_{t}^{\varepsilon}\left(\bar{k}_{5}\right):\right] \\
=12 \prod_{i=1}^{5} \frac{f\left(\varepsilon k_{i}\right)^{2}}{\left|k_{i}\right|^{2}} \delta_{k_{i}=-\bar{k}_{i}} e^{-|s-\bar{s}|\left(\left|k_{1}\right|^{2}+\left|k_{2}\right|^{2}+\left|k_{3}\right|^{2}\right)} \\
\quad+72 \prod_{i=1}^{5} \frac{f\left(\varepsilon k_{i}\right)^{2}}{\left|k_{i}\right|^{2}} \delta_{k_{1}=-\bar{k}_{1}} \delta_{k_{2}=-\bar{k}_{2}} \delta_{k_{3}=-\bar{k}_{4}} \delta_{k_{4}=-\bar{k}_{3}} \delta_{k_{5}=-\bar{k}_{5}} \\
\quad \times e^{-|s-\bar{s}|\left(\left|k_{1}\right|^{2}+\left|k_{2}\right|^{2}\right)-|t-s|\left|k_{3}\right|^{2}-|t-\bar{s}|\left|k_{4}\right|^{2}} \\
\quad+36 \prod_{i=1}^{5} \frac{f\left(\varepsilon k_{i}\right)^{2}}{\left|k_{i}\right|^{2}} \delta_{k_{1}=-\bar{k}_{1}} \delta_{k_{2}=-\bar{k}_{4}} \delta_{k_{3}=-\bar{k}_{5}} \delta_{k_{4}=-\bar{k}_{3}} \delta_{k_{5}=-\bar{k}_{2}} \\
\quad \times e^{-|s-\bar{s}|\left|k_{1}\right|^{2}-|t-s|\left(\left|k_{2}\right|^{2}+\left|k_{3}\right|^{2}\right)-|t-\bar{s}|\left(\left|k_{4}\right|^{2}+\left|k_{5}\right|^{2}\right)} .
\end{aligned}
$$

Then

$$
\mathbb{E}\left[\left|\Delta_{q} I_{t}^{1}\right|^{2}\right]=E_{t}^{1,1}+E_{t}^{1,2}+E_{t}^{1,3},
$$

where

$$
\begin{aligned}
E_{t}^{1,1}= & 12 \int_{[0, t]^{2}} \mathrm{~d} s \mathrm{~d} \bar{s} \theta\left(2^{-q} k\right)^{2} \sum_{\substack{k \\
k_{12345}=k}} \prod_{i=1}^{5} \frac{f\left(\varepsilon k_{i}\right)^{2}}{\left|k_{i}\right|^{2}} \\
& \times e^{-\left|k_{123}\right|^{2}|t-s|} e^{-\left(\left|k_{1}\right|^{2}+\left|k_{2}\right|^{2}+\left|k_{3}\right|^{2}\right)|s-\bar{s}|}, \\
E_{t}^{1,2}= & 72 \int_{[0, t]^{2}} \mathrm{~d} s \mathrm{~d} \bar{s} \sum_{k} \theta\left(2^{-q} k\right)^{2} \prod_{i=1}^{5} \frac{f\left(\varepsilon k_{i}\right)^{2}}{\left|k_{i}\right|^{2}} \\
& \times e^{-\left(\left|k_{123}\right|^{2}+\left|k_{3}\right|^{2}\right)|t-s|} e^{-\left(\left|k_{124}\right|^{2}+\left|k_{4}\right|^{2}\right)|t-\bar{s}|} e^{-|s-\bar{s}|\left(\left|k_{1}\right|^{2}+\left|k_{2}\right|^{2}\right)}
\end{aligned}
$$

and

$$
\begin{aligned}
E_{t}^{1,3}= & 36 \int_{0}^{t} \mathrm{~d} s \int_{0}^{t} \mathrm{~d} \bar{s} \sum_{\substack{k \neq 0, k_{i} \neq 0 \\
k_{12345}=k}} \theta\left(2^{-q} k\right)^{2} \prod_{i=1}^{5} \frac{f\left(\varepsilon k_{i}\right)^{2}}{\left|k_{i}\right|^{2}} \\
& \times e^{-\left(\left|k_{123}\right|^{2}+\left|k_{2}\right|^{2}+\left|k_{3}\right|^{2}\right)|t-s|} e^{-\left(\left|k_{145}\right|^{2}+\left|k_{5}\right|^{2}+\left|k_{4}\right|^{2}\right)|t-\bar{s}|} e^{-|s-\bar{s}|\left|k_{1}\right|^{2}},
\end{aligned}
$$


Estimation of $E_{t}^{1,1}$. Let us rewrite it in a form easier to handle:

$$
\begin{aligned}
E_{t}^{1,1}= & 12 \int_{[0, t]^{2}} \mathrm{~d} s \mathrm{~d} \bar{s} \sum_{\substack{k, k \neq 0 \\
k_{1}+k_{2}+l=k \\
l_{1}+l_{2}+l_{3}=l \\
k_{i} \neq 0, l_{i} \neq 0}} \theta\left(2^{-q} k\right)^{2} \\
& \times \prod_{i=1}^{2} \frac{f\left(\varepsilon k_{i}\right)^{2}}{\left|k_{i}\right|^{2}} \prod_{i=1}^{3} \frac{f\left(\varepsilon l_{i}\right)^{2}}{\left|l_{i}\right|^{2}} e^{-|l|^{2}|t-s|} e^{-\left(\left|l_{1}\right|^{2}+\left|l_{2}\right|^{2}+\left|l_{3}\right|^{2}\right)|s-\bar{s}|} .
\end{aligned}
$$

Thanks to the symmetries of this term, we can always assume that $\left|k_{1}\right|=\max \left(\left|k_{i}\right|\right)$ and $l_{1}=\max \left(\left|l_{i}\right|\right)$.

For $l=0$, we have

$$
\begin{aligned}
\int_{[0, t]^{2}} \mathrm{~d} s \mathrm{~d} \bar{s} \sum_{\substack{k, k \neq 0 \\
k_{1}+k_{2}=k \\
l_{1}+l_{2}+l_{3}=0 \\
k_{i} \neq 0, l_{i} \neq 0}} \theta\left(2^{-q} k\right)^{2} \prod_{i=1}^{2} \frac{f\left(\varepsilon k_{i}\right)^{2}}{\left|k_{i}\right|^{2}} \\
\times \prod_{i=1}^{3} \frac{f\left(\varepsilon l_{i}\right)^{2}}{\left|l_{i}\right|^{2}} e^{-\left(\left|l_{1}\right|^{2}+\left|l_{2}\right|^{2}+\left|l_{3}\right|^{2}\right)|s-\bar{s}|} \\
\lesssim \int_{[0, t]^{2}} \mathrm{~d} s \mathrm{~d} \bar{s} \sum_{k \neq 0} \theta\left(2^{-q} k\right)^{2}|k|^{-1+\eta} \sum_{k_{2} \neq 0}|k|^{-3-\eta} \\
\quad \times \sum_{l_{2} \neq 0, l_{3} \neq 0}\left|l_{2}\right|^{-4+\eta}\left|l_{3}\right|^{-4+\eta}|s-\bar{s}|^{-1+\eta} \\
\lesssim 2^{q(2+\eta)} t .
\end{aligned}
$$

Let us assume that $|l|=\max \left(|l|,\left|k_{1}\right|\right)$ as we have the following estimate $\left|l_{1}\right|^{-1} \lesssim|l|^{-1}$. The following bound holds:

$$
\begin{aligned}
\int_{[0, t]^{2}} \mathrm{~d} s & \mathrm{~d} \bar{s} \sum_{k \neq 0} \theta\left(2^{-q} k\right)^{2}|k|^{-1+\eta} \sum_{\substack{k_{1} k_{2} \neq 0 \\
l_{2}, l_{3} \neq 0}}\left(\left|k_{1}\right|\left|k_{2}\right|\right)^{-4+9 \eta / 2} \\
& \times(|t-s||s-\bar{s}|)^{-1+\eta}\left(\left|l_{2}\right|\left|l_{3}\right|\right)^{-3-\eta} \\
\lesssim & t^{\eta} 2^{q(2+\eta)} .
\end{aligned}
$$

The case in which $\left|k_{1}\right|=\max \left(|l|,\left|k_{1}\right|\right)$ is quite similar, and the conclusion holds for $E_{t}^{1,1}$.

Estimation of $E_{t}^{1,2}$. This term is symmetric in $k_{1}, k_{2}$ and in $k_{3}, k_{4}$. Hence we can assume that $\left|k_{1}\right| \geq\left|k_{2}\right|$ and $\left|k_{3}\right| \geq\left|k_{4}\right|$ First, let us assume that $\left|k_{5}\right|=$ 
$\max \left\{\left|k_{i}\right|\right\}$. Then

$$
\begin{aligned}
E_{t}^{1,2} \lesssim & \sum_{\substack{k \\
k_{12345}=k}} \theta\left(2^{-q} k\right)^{2} \int_{0}^{t} \mathrm{~d} s \int_{0}^{s} \mathrm{~d} \bar{s}(|t-s||s-\bar{s}|)^{-1+\eta} \\
& \times\left|k_{1}\right|^{-4+2 \eta}\left|k_{2}\right|^{-2}\left|k_{3}\right|^{-4+2 \eta}\left|k_{4}\right|^{-2}\left|k_{5}\right|^{-\left(1+\eta^{\prime}\right)}|k|^{-\left(1-\eta^{\prime}\right)} \\
\lesssim & t^{\eta} \sum_{k} \theta\left(2^{-q} k\right)^{2}|k|^{-\left(1-\eta^{\prime}\right)} \sum_{k_{12345}=k}\left|k_{1}\right|^{-7 / 2+2 \eta}\left|k_{2}\right|^{-3-\eta^{\prime} / 2} \\
& \times\left|k_{3}\right|^{-7 / 2+2 \eta}\left|k_{4}\right|^{-3-\eta^{\prime} / 2} \\
\lesssim & t^{\eta} 2^{\left(2+\eta^{\prime}\right) q}
\end{aligned}
$$

for $\eta$ small enough.

Then assume that $\max \left\{\left|k_{i}\right|\right\}=\left|k_{1}\right|$ :

$$
\begin{aligned}
& E_{t}^{1,2} \lesssim t^{\delta} \sum_{\substack{k \\
k_{12345}=k}} \theta\left(2^{-q} k\right)^{2}\left|k_{1}\right|^{-4+2 \eta}\left|k_{2}\right|^{-2}\left|k_{3}\right|^{-3+\eta^{\prime}}\left|k_{4}\right|^{-3+\eta^{\prime}}\left|k_{5}\right|^{-2} \\
& \times \int_{0}^{t} \mathrm{~d} s \int_{0}^{s} \mathrm{~d} \bar{s}|t-s|^{-1+\eta^{\prime}}|s-\bar{s}|^{-1+\eta} \\
& \lesssim t^{\eta^{\prime}} \sum_{k} \theta\left(2^{-q} k\right)^{2}|k|^{-1+\eta^{\prime \prime}}\left|k_{2}\right|^{-3-\eta^{\prime \prime}} \\
& \quad \times\left|k_{3}\right|^{-7 / 2+\left(2 \eta+\eta^{\prime \prime}+\eta^{\prime}\right) / 2}\left|k_{4}\right|^{-7 / 2+\left(2 \eta+\eta^{\prime \prime}+\eta^{\prime}\right) / 2}\left|k_{5}\right|^{-3-\eta^{\prime \prime}} \\
& \lesssim t^{\delta} 2^{\left(2+\eta^{\prime}\right) q} .
\end{aligned}
$$

For $\max \left\{\left|k_{i}\right|\right\}=\left|k_{3}\right|$,

$$
\begin{aligned}
E_{t}^{1,2} & \lesssim t^{\delta} \sum_{\substack{k \\
k_{12345}=k}} \theta\left(2^{-q} k\right)^{2}\left|k_{1}\right|^{-4+\eta}\left|k_{2}\right|^{-2}\left|k_{3}\right|^{-4+\eta^{\prime}}\left|k_{4}\right|^{-2}\left|k_{5}\right|^{-2} \\
& \lesssim t^{\delta} \sum_{\substack{k \\
k_{12345}=k}} \theta\left(2^{-q} k\right)^{2}\left|k_{1}\right|^{-3+\eta+1 / 4}\left|k_{2}\right|^{-3+1 / 4}|k|^{-1+\eta^{\prime}}\left|k_{4}\right|^{-3+1 / 4}\left|k_{5}\right|^{-3+1 / 4} \\
& \lesssim t^{\delta} 2^{\left(2+\eta^{\prime}\right) q}
\end{aligned}
$$

hence there exist $\delta>0$ and $v>0$ such that

$$
E_{t}^{1,2} \lesssim t^{\delta} 2^{(2+v) q}
$$

Estimation of $E_{t}^{1,3}$. Let us deal with this last term. Here, the symmetries are in $k_{2}, k_{3}$ and $k_{4}, k_{5}$. Then we can suppose that $\left|k_{2}\right| \geq\left|k_{3}\right| \geq$ and $\left|k_{4}\right| \geq\left|k_{5}\right|$. Furthermore, the role of $k_{2}, k_{3}$ and $k_{4}, k_{5}$ are symmetrical, then we can assume that 
$\left|k_{1}\right| \geq\left|k_{4}\right|$, and we have

$$
\begin{aligned}
E_{t}^{1,3}= & \int_{[0, t]^{2}} \mathrm{~d} s \mathrm{~d} \bar{s} \sum_{\substack{k \neq 0, k_{i} \neq 0 \\
k_{12345}=k}} \theta\left(2^{-q} k\right)^{2} \prod_{i=1}^{5} \frac{f\left(\varepsilon k_{i}\right)^{2}}{\left|k_{i}\right|^{2}} \\
& \times e^{-\left(\left|k_{123}\right|^{2}+\left|k_{2}\right|^{2}+\left|k_{3}\right|^{2}\right)|t-s|} e^{-\left(\left|k_{145}\right|^{2}+\left|k_{5}\right|^{2}+\left|k_{4}\right|^{2}\right)|t-\bar{s}|} e^{-|s-\bar{s}|\left|k_{1}\right|^{2}} .
\end{aligned}
$$

If $\left|k_{1}\right|=\max \left(\left|k_{i}\right|\right)$, then

$$
\begin{aligned}
& \lesssim \int_{[0, t]^{2}} \mathrm{~d} s \mathrm{~d} \bar{s}(|t-s||t-\bar{s}|)^{-1+\eta} \\
& \times \sum_{\substack{k \neq 0, k_{i} \neq 0 \\
k_{12345}=k}} \theta\left(2^{-q} k\right)^{2}|k|^{-1+\eta}\left(\left|k_{2}\right|\left|k_{3}\right|\left|k_{3}\right|\left|k_{4}\right|\right)^{-7 / 4+3 \eta / 4} \\
& \lesssim 2^{q(2+\eta)} t^{\eta} .
\end{aligned}
$$

If $\left|k_{2}\right|=\max \left(\left|k_{i}\right|\right)$, then

$$
\begin{aligned}
& \lesssim 2 \int_{0}^{t} \int_{0}^{s} \mathrm{~d} s \mathrm{~d} \bar{s}(|t-s||s-\bar{s}|)^{-1+\eta} \\
& \times \sum_{\substack{k \neq 0, k_{i} \neq 0 \\
k_{12345}=k}} \theta\left(2^{-q} k\right)^{2}|k|^{-1+\eta}\left(\left|k_{1}\right|\left|k_{3}\right|\left|k_{3}\right|\left|k_{4}\right|\right)^{-7 / 4+3 \eta / 4} \\
& \lesssim t^{\eta} 2^{q(2+\eta)} .
\end{aligned}
$$

\section{APPENDIX: A COMMUTATION LEMMA}

We give the proof of Lemma 2.5. This proof is from [18], Lemmas 5.3.20 and 5.5.7. In fact, we give a stronger result, and apply it with $\varphi(k)=\exp \left(-|k|^{2} / 2\right)$.

LemMA A.1. Let $\alpha<1$ and $\beta \in \mathbb{R}$. Let $\varphi \in \mathcal{S}$, let $u \in C^{\alpha}$, and $v \in C^{\beta}$. Then for every $\varepsilon>0$ and every $\delta \geq-1$ we have

$$
\left\|\varphi(\varepsilon \mathscr{D}) \pi_{<}(u, v)-\pi_{<}(u, \varphi(\varepsilon \mathscr{D}) v)\right\|_{\alpha+\beta+\delta} \lesssim \varepsilon^{-\delta}\|u\|_{\alpha}\|v\|_{\beta},
$$

where

$$
\varphi(\mathscr{D}) u=\mathscr{F}^{-1}(\varphi \mathscr{F} u)
$$

PROOF. We define for $j \geq-1$

$$
S_{j-1} u=\sum_{i=-1}^{j-2} \Delta_{i} u
$$


and

$$
\begin{aligned}
\varphi(\varepsilon \mathscr{D}) \pi_{<}(u, v)-\pi_{<}(u, \varphi(\varepsilon \mathscr{D}) v) \\
=\sum_{j \geq-1}\left(\varphi(\varepsilon \mathscr{D})\left(S_{j-1} u \Delta_{j} v\right)-S_{j-1} u \Delta_{j} \varphi(\varepsilon \mathscr{D}) v\right),
\end{aligned}
$$

where every term of this series has a Fourier transform with support in an annulus of the form $2^{j} \mathcal{A}$. Lemma 2.69 in [1] implies that it is enough to control the $L^{\infty}$ norm of each term. Let $\psi \in \mathcal{D}$ with support in an annulus be such that $\psi \equiv 1$ on $\mathcal{A}$. We have

$$
\begin{aligned}
\varphi(\varepsilon \mathscr{D}) & \left(S_{j-1} u \Delta_{j} v\right)-S_{j-1} u \Delta_{j} \varphi(\varepsilon \mathscr{D}) v \\
= & \left(\psi\left(2^{-j} \cdot\right) \varphi(\varepsilon \cdot)\right)(\mathscr{D})\left(S_{j-1} u \Delta_{j} v\right)-S_{j-1} u\left(\psi\left(2^{-j} \cdot\right) \varphi(\varepsilon \cdot)\right)(\mathscr{D}) \Delta_{j} v \\
= & {\left[\left(\psi\left(2^{-j} \cdot\right) \varphi(\varepsilon \cdot)\right)(\mathscr{D}), S_{j-1} u\right] \Delta_{j} v, }
\end{aligned}
$$

where $\left[\left(\psi\left(2^{-j} \cdot\right) \varphi(\varepsilon \cdot)\right)(\mathscr{D}), S_{j-1} u\right]$ denotes the commutator. In the proof of Lemma 2.97 in [1], it is shown that writing the Fourier multiplier as a convolution operator and applying a first-order Taylor expansion and then Young's inequality yields to

$$
\begin{aligned}
& \left\|\left[\left(\psi\left(2^{-j} \cdot\right) \varphi(\varepsilon \cdot)\right)(\mathscr{D}), S_{j-1} u\right] \Delta_{j} v\right\|_{L^{\infty}} \\
& \quad \lesssim \sum_{\eta \in \mathbb{N}^{d}:|\eta|=1}\left\|x^{\eta} \mathscr{F}^{-1}\left(\psi\left(2^{-j} \cdot\right) \varphi(\varepsilon \cdot)\right)\right\|_{L^{1}}\left\|\partial^{\eta} S_{j-1} u\right\|_{L^{\infty}}\left\|\Delta_{j} v\right\|_{L^{\infty}}
\end{aligned}
$$

Now $\mathscr{F}^{-1}\left(f\left(2^{-j} \cdot\right) g(\varepsilon \cdot)\right)=2^{j d} \mathscr{F}^{-1}\left(f g\left(\varepsilon 2^{j} \cdot\right)\right)\left(2^{j} \cdot\right)$ for every $f, g$, and thus we have for every multi-index $\eta$ of order one:

$$
\begin{aligned}
& \left\|x^{\eta} \mathscr{F}^{-1}\left(\psi\left(2^{-j} \cdot\right) \varphi(\varepsilon \cdot)\right)\right\|_{L^{1}} \\
& \leq 2^{-j}\left\|\mathscr{F}^{-1}\left(\left(\partial^{\eta} \psi\right)\left(2^{-j} \cdot\right) \varphi(\varepsilon \cdot)\right)\right\|_{L^{1}} \\
& +\varepsilon\left\|\mathscr{F}^{-1}\left(\psi\left(2^{-j} \cdot\right) \partial^{\eta} \varphi(\varepsilon \cdot)\right)\right\|_{L^{1}} \\
& =2^{-j}\left\|\mathscr{F}^{-1}\left(\left(\partial^{\eta} \psi\right) \varphi\left(\varepsilon 2^{j} \cdot\right)\right)\right\|_{L^{1}}+\varepsilon\left\|\mathscr{F}^{-1}\left(\psi \partial^{\eta} \varphi\left(\varepsilon 2^{j} \cdot\right)\right)\right\|_{L^{1}} \\
& \lesssim 2^{-j}\left\|(1+|\cdot|)^{2 d} \mathscr{F}^{-1}\left(\left(\partial^{\eta} \psi\right) \varphi\left(\varepsilon 2^{j} \cdot\right)\right)\right\|_{L^{\infty}} \\
& +\varepsilon\left\|(1+|\cdot|)^{2 d} \mathscr{F}^{-1}\left(\psi \partial^{\eta} \varphi\left(\varepsilon 2^{j} \cdot\right)\right)\right\|_{L^{\infty}} \\
& =2^{-j}\left\|\mathscr{F}^{-1}\left((1-\Delta)^{d}\left(\left(\partial^{\eta} \psi\right) \varphi\left(\varepsilon 2^{j} \cdot\right)\right)\right)\right\|_{L^{\infty}} \\
& +\varepsilon\left\|\mathscr{F}^{-1}\left((1-\Delta)^{d}\left(\psi \partial^{\eta} \varphi\left(\varepsilon 2^{j} \cdot\right)\right)\right)\right\|_{L^{\infty}} \\
& \lesssim 2^{-j}\left\|(1-\Delta)^{d}\left(\left(\partial^{\eta} \psi\right) \varphi\left(\varepsilon 2^{j} \cdot\right)\right)\right\|_{L^{\infty}} \\
& +\varepsilon\left\|(1-\Delta)^{d}\left(\psi \partial^{\eta} \varphi\left(\varepsilon 2^{j} \cdot\right)\right)\right\|_{L^{\infty}},
\end{aligned}
$$


where the last step follows because $\psi$ has compact support. For $j$ satisfying $\varepsilon 2^{j} \geq$ 1, we obtain

$$
\begin{aligned}
\left\|x^{\eta} \mathscr{F}^{-1}\left(\varphi(\varepsilon \cdot) \psi\left(2^{-j} \cdot\right)\right)\right\|_{L^{1}} & \\
& \lesssim\left(\varepsilon+2^{-j}\right)\left(\varepsilon 2^{j}\right)^{2 d} \sum_{\eta:|\eta| \leq 2 d+1}\left\|\partial^{\eta} \varphi\left(\varepsilon 2^{j} \cdot\right)\right\|_{L^{\infty}(\operatorname{supp}(\psi))},
\end{aligned}
$$

where we used that $\psi$ and all its partial derivatives are bounded, and where $L^{\infty}(\operatorname{supp}(\psi))$ means that the supremum is taken over the values of $\partial^{\eta} \varphi\left(\varepsilon 2^{j} \cdot\right)$ restricted to $\operatorname{supp}(\psi)$. Now $\varphi$ is a Schwartz function and, therefore, it decays faster than any polynomial. Hence there exists a ball $\mathcal{B}_{\delta}$ such that for all $x \notin \mathcal{B}_{\delta}$ and all $|\eta| \leq 2 d+1$ we have

$$
\left|\partial^{\eta} \varphi(x)\right| \leq|x|^{-2 d-1-\delta}
$$

Let $j_{0} \in \mathbb{N}$ be minimal such that $2^{j_{0}} \varepsilon \mathcal{A} \cap \mathcal{B}_{\delta}=\varnothing$ and $\varepsilon 2^{j_{0}} \geq 1$. Then the combination of (A.1), (A.3) and (A.4) shows that, for all $j \geq j_{0}$,

$$
\begin{aligned}
\left\|\left[\left(\psi\left(2^{-j} \cdot\right) \varphi(\varepsilon \cdot)\right)(\mathscr{D}), S_{j-1} u\right] \Delta_{j} v\right\|_{L^{\infty}} \\
\lesssim\left(\varepsilon+2^{-j}\right)\left(\varepsilon 2^{j}\right)^{2 d} \sum_{\eta:|\eta| \leq 2 d+1}\left\|\left(\partial^{\eta} \varphi\right)\left(\varepsilon 2^{j} \cdot\right)\right\|_{L^{\infty}(\operatorname{supp}(\psi))} \\
\quad \times 2^{j(1-\alpha)}\|u\|_{\alpha} 2^{-j \beta}\|v\|_{\beta} \\
\lesssim\left(\varepsilon+2^{-j}\right)\left(\varepsilon 2^{j}\right)^{2 d}\left(\varepsilon 2^{j}\right)^{-2 d-1-\delta} 2^{j(1-\alpha-\beta)}\|u\|_{\alpha}\|v\|_{\beta} \\
\lesssim\left(1+\left(\varepsilon 2^{j}\right)^{-1}\right) \varepsilon^{-\delta} 2^{-j(\alpha+\beta+\delta)}\|u\|_{\alpha}\|v\|_{\beta} .
\end{aligned}
$$

Here, we used that $\alpha<1$ in order to obtain $\left\|\partial^{\eta} S_{j-1} u\right\|_{L^{\infty}} \lesssim 2^{j(1-\alpha)}\|u\|_{L^{\infty}}$. Since $\varepsilon 2^{j} \geq 1$, we have shown the desired estimate for $j \geq j_{0}$. On the other side, Lemma 2.97 in [1] implies that, for every $j \geq-1$,

$$
\begin{aligned}
\left\|\left[\varphi(\varepsilon \mathscr{D}), S_{j-1} u\right] \Delta_{j} v\right\|_{L^{\infty}} & \lesssim \varepsilon \max _{\eta \in \mathbb{N}^{d}:|\eta|=1}\left\|\partial^{\eta} S_{j-1} u\right\|_{L^{\infty}}\left\|\Delta_{j} v\right\|_{L^{\infty}} \\
& \lesssim \varepsilon 2^{j(1-\alpha-\beta)}\|u\|_{\alpha}\|v\|_{\beta} .
\end{aligned}
$$

Hence, we obtain the bound for $j<j_{0}$, that is, for $j$ satisfying $2^{j} \varepsilon \lesssim 1$,

$$
\begin{aligned}
\left\|\left[\varphi(\varepsilon \mathscr{D}), S_{j-1} u\right] \Delta_{j} v\right\|_{L^{\infty}} & \lesssim\left(\varepsilon 2^{j}\right)^{1+\delta} \varepsilon^{-\delta} 2^{-j(\alpha+\beta+\delta)}\|u\|_{\alpha}\|v\|_{\beta} \\
& \lesssim \varepsilon^{-\delta} 2^{-j(\alpha+\beta+\delta)}\|u\|_{\alpha}\|v\|_{\beta},
\end{aligned}
$$

where we used that $\delta \geq-1$. This completes the proof. 


\section{REFERENCES}

[1] Bahouri, H., Chemin, J.-Y. and Danchin, R. (2011). Fourier Analysis and Nonlinear Partial Differential Equations. Grundlehren der Mathematischen Wissenschaften [Fundamental Principles of Mathematical Sciences] 343. Springer, Heidelberg. MR2768550

[2] Bertini, L., Jona-Lasinio, G. and Parrinello, C. (1993). Stochastic quantization, stochastic calculus and path integrals: Selected topics. Progr. Theoret. Phys. Suppl. 111 83-113. MR1263920

[3] Bertini, L., Presutti, E., Rüdiger, B. and SaAda, E. (1993). Dynamical fluctuations at the critical point: Convergence to a nonlinear stochastic PDE. Teor. Veroyatn. Primen. 38 689-741. MR1317994

[4] Da Prato, G. and Debussche, A. (2003). Strong solutions to the stochastic quantization equations. Ann. Probab. 31 1900-1916. MR2016604

[5] Garsia, A. M., Rodemich, E. and Rumsey, H. JR. (1970/1971). A real variable lemma and the continuity of paths of some Gaussian processes. Indiana Univ. Math. J. 20 565-578. MR0267632

[6] Glimm, J. and Jaffe, A. (1981). Quantum Physics: A Functional Integral Point of View. Springer, New York. MR0628000

[7] Gubinelli, M. (2004). Controlling rough paths. J. Funct. Anal. 216 86-140. MR2091358

[8] Gubinelli, M., Imkeller, P. and Perkowski, N. (2015). Paracontrolled distributions and singular PDEs. Forum Math., Pi 3 e6.

[9] HaIrer, M. (2013). Solving the KPZ equation. Ann. of Math. 178 559-664.

[10] HAIRER, M. (2014). A theory of regularity structures. Invent. Math. 198 269-504. MR3274562

[11] Hairer, M. and Weber, H. (2015). Large deviations for white-noise driven, nonlinear stochastic PDEs in two and three dimensions. Ann. Fac. Sci. Toulouse Math. (6) 24 55-92 MR3325951.

[12] Janson, S. (1997). Gaussian Hilbert Spaces. Cambridge Univ. Press, Cambridge.

[13] Jona-Lasinio, G. and MitTer, P. K. (1985). On the stochastic quantization of field theory. Comm. Math. Phys. 101 409-436. MR0815192

[14] Jona-Lasinio, G. and Mitter, P. K. (1990). Large deviation estimates in the stochastic quantization of $\phi_{2}^{4}$. Comm. Math. Phys. 130 111-121. MR1055688

[15] MourRat, J. C. and WeBer, H. (2017). Convergence of the two-dimensional dynamic IsingKac model to $\Phi_{4}^{2}$. Comm. Pure Appl. Math. 70 717-812 MR3628883.

[16] Mourrat, J.-C. and Weber, H. (2017). Global well-posedness of the dynamic $\Phi^{4}$ model in the plane. Ann. Probab. 45 2398-2476. MR3693966

[17] Mourrat, J.-C. and Weber, H. (2017). Global well-posedness of the dynamic $\Phi_{4}^{3}$ model on the torus. Preprint. Available at arXiv:1601.01234.

[18] PerkowsKi, N. (2013). Studies of robustness in stochastic analysis and mathematical finance. Ph.D. thesis, Humboldt Univ. Berlin.

LABORATOIRE J.A.DIEUDONNÉ

UMR CNRS-UNS 7351

Université de NicE SOPHIA-ANTIPOLIS

PARC VALROSE

06108 NiCE CEDEX 2

FRANCE

E-MAIL: remi.catellier@unice.fr
INSTITUT FÜR MATHEMATIK

AG STOCHASTIK UND FINANZMATHEMATIK TECHNISCHE UNIVERSITÄT BERLIN

BERLIN 10623

GERMANY

E-MAIL: khalil.chouk@gmail.com 